Bruno Galelli Chieregatti

\title{
AplicaÇÃo do Método Adjunto EM ESCOAMENTOS VISCOSOS INCOMPRESSÍVEIS e Periódicos. Estudo de Caso: SPlitTer Plate
}

\author{
Dissertação apresentada à Escola Poli- \\ técnica da Universidade de São Paulo \\ para obtenção do Título de Mestre em \\ Engenharia Mecânica.
}




\section{Bruno Galelli Chieregatti}

\section{AplichÇÃo do MÉtodo Adjunto EM ESCOAMENTOS VISCOSOS INCOMPRESSÍVEIS e Periódicos. Estudo de Caso: SPLITTER Plate}

Dissertação apresentada à Escola Politécnica da Universidade de São Paulo para obtenção do Título de Mestre em Engenharia Mecânica.

Área de concentração:

Engenharia Mecânica

Orientador:

Prof. Dr. Ernani Vitillo Volpe 


\section{Ficha Catalográfica}

Chieregatti, Bruno Galelli

Aplicação do Método Adjunto em Escoamentos Viscosos Incompressíveis e Periódicos. Estudo de Caso: Splitter Plate. São Paulo, 2012. 128 p.

Dissertação (Mestrado) — Escola Politécnica da Universidade de São Paulo. Departamento de Engenharia Mecânica.

1. Método Adjunto. 2. Otimização. 3.Aerodinâmica. 4. Dinâmica dos Fluidos Computacional. 5. Condições de Contorno. I. Universidade de São Paulo. Escola Politécnica. Departamento de Engenharia Mecânica. II. Aplicação do Método Adjunto em Escoamentos Viscosos Incompressíveis e Periódicos. Estudo de Caso: Splitter Plate. 
Aos meus pais, Júnior e Alice, que sempre me apoiaram nos estudos.

E à minha futura esposa, Talita, pelo amor e pela presença durante o desenvolvimento do meu mestrado. 


\section{AgradeCimentos}

Eu vejo minha vida formada por três grandes pilares: A família de criação, a família que irei formar e minha família profissional.

Na família em formação, agradeço a minha futura esposa Talita, que acompanhou este trabalho desde o começo em 2009. Através do meu exemplo de estudo, ela também está finalizando um ciclo de formação, obtendo assim seu bacharelado em administração. Juntos somos fortes e nos manteremos assim pelo resto de nossas vidas. Amo você!

Pela minha família de criação, agradeço meus pais, Júnior e Alice, por me ensinarem desde pequeno o valor do estudo e os frutos que irei colher por ter me dedicado a educação, sempre buscando uma ótima formação profissional. Também agradeço e dedico este trabalho a minha irmã Bianca, a qual me tomou como exemplo e buscou sua formação de qualidade na faculdade.

Aos meus grandes amigos, André Marguti, Diego Carreras e Suyami Maruyama, um grande agradecimento por estarem comigo nas horas boas e ruins, sempre dispostos a me ajudar no que precisasse.

Na família profissional, agradeço toda equipe de pesquisa do NDF que colaborou neste trabalho, mencionando os professores Ernani Volpe, Bruno Carmo e Julio Meneghini, o Doutor Rafael Gioria, os mestres Marcelo Hayashi e Marco Ceze e os mestrandos Saulo Maciel, João Brasil Lima e Stérgios Tsiloufas.

Agradeço a empresa que trabalho, a Estudos Técnicos e Projetos (ETEP) que me cedeu tempo para que eu pudesse assistir as aulas e para estar na Poli produzindo minha pesquisa. Representando a empresa, gostaria de agradecer aos grandes Engenheiros Hildebrando Vasconcellos e José Luiz Vieira de Toledo Prado pelo apoio.

A todos, meu sincero MUITO OBRIGADO! 
"Veni, vidi, vici! - Vim, vi e venci!"

(Julio César) 


\section{RESUMO}

O presente trabalho é o início de um estudo da aplicação do método de otimização conhecido como adjunto em escoamentos incompressíveis, viscosos e periódicos, envolvendo um problema de bastante interesse: a análise da aplicação de splitter plates em cilindros de seção circular.

Conhecido por sua simplicidade, o splitter plate, que consiste em uma placa plana alinhada ao escoamento e colocada a jusante do corpo, é um dispositivo efetivo na mudança de comportamento da esteira de vórtices de Von Kárman.

A partir da revisão bibliográfica foi possível entender a dinâmica do escoamento, proporcionando uma calibração dos modelos numéricos. Complementando esta etapa, foi efetuada uma análise da qualidade das malhas computacionais.

Através de uma geração de diversas malhas computacionais, o espaço de soluções foi explorado buscando encontrar o mínimo arrasto para diversos comprimentos de splitter plate e diferentes números de Reynolds $(R e)$.

Foi observada a influência da placa na formação da esteira de vórtices, obtendo uma redução dos coeficientes de força do cilindro. Com esses dados, foi possível desenvolver o método de otimização voltado para análise do gradiente de sensibilidade conhecido como método adjunto baseado nas equações de Navier Stokes utilizando o problema descrito como base para validação dos resultados.

A abordagem do método adjunto caracteriza-se pela busca dos extremos de funções conhecidas como medidas de mérito. Essas funções podem ser integrais de sustentação e arrasto por exemplo. Na literatura, o método adjunto é apresentado como possuindo duas grandes vantagens: a primeira é a imposição das equações do escoamento como restrições do problema, o que sempre confinará as variações da medida de mérito dentro do universo de soluções realizáveis; já a segunda é conseqüência da primeira, pois as restrições permitem uma simplificação no cálculo do gradiente de sensibilidade, reduzindo o custo computacional.

Para o cálculo do gradiente de sensibilidade, o objetivo é otimizar o arrasto do cilindro sob efeito do splitter plate variando os parâmetros de controle (comprimento e posicionamento do splitter plate). A direção de busca e o cálculo do passo da geometria são obtidos a partir da relação entre a solução numérica do escoamento e as variáveis adjuntas calculadas.

Nesta dissertação, será apresentada a pesquisa bibliográfica, os resultados do método "tentativa e erro", a formulação do método adjunto baseado nas equações de Navier Stokes e um exemplo de sua solução numérica, demonstrando sua existência.

Palavras chave: adjunto, otimização, splitter plates, CFD, cilindros. 


\section{ABSTRACT}

The report is the beginning of a research about the aplication of the so called adjoint method in the optimization of incompressive, viscous and periodic flows. The study involves a problem of great interest: an analysis of the implementation of splitter plates in the flow around cylinders with circular section.

Widely recognized for its simplicity, the splitter plate consists of a flat plate, which is placed in the wake of a cylinder, in the stream-wise direction, and it works by changing the way the shear layers interact with one another.

Based on a literature survey, it is possible to understand the physics of this class of flows. As a better result, one learns what to expect from the numerical solutions and hence, one can calibrate its parameters. Moreover, we study the best configuration of the computational mesh, thus reducing the computacional cost.

After the generation of meshes, the universe of solutions was explored to find the minimum drag for various lenghts of splitter plate and Reynolds number $(R e)$.

The influence of the plate in the interation of the shear layers was observed in the reduction of drag coefficient. These results form a the basis for comparison, upon one can develop the optimization by the adjoint method.

The adjoint method can be used to search the extrema of objective functionals. These functionals can be the lift and drag integrals for example. The theory presents two advantages to the method: first, the imposing the equations that govern the flow as variational contraints one limits the variations to the universe of realizable solutions; second, these constrains simplify the computation of the sentivity gradient, by reducing its computational cost.

To compute the sensitivity gradient, the objective functional can be defined as the average drag coefficent of the circular cylinder with a splitter plate. The control parameters are the lenght of the plate and the distance between it and the body, which known as gap. The search direction and the variation of geometry can be obtained by the relationship between the solutions to the flow and the adjoint equations.

This final report shows the literature survey, the results of "trial and error" method and the formulation and one result of adjoint equations based on the incompressible Navier-Stokes equations.

Key words: adjoint, optimization, splitter plates, CFD, cylinder. 


\title{
SUMÁRIO
}

\section{Lista de Figuras}

\author{
Lista de Tabelas
}

\section{Acrônimos}

1 Introdução 1

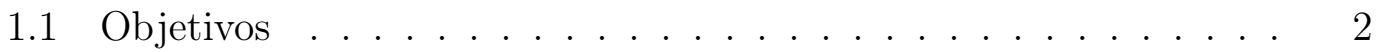

1.2 Escoamento ao redor de cilindros . . . . . . . . . . . . . . 4

1.3 O Splitter Plate . . . . . . . . . . . . . . . . . 7

1.4 Revisão Bibliográfica . . . . . . . . . . . . . . . . . . . . 7

2 Metodologia e fundamentos teóricos $\quad 17$

2.1 Introdução . . . . . . . . . . . . . . . . . . . . . 17

2.2 Modelo Matemático do Escoamento . . . . . . . . . . . . . . . 17

2.3 Solução Numérica do Escoamento . . . . . . . . . . . . . . . . . . 18

2.3.1 Método dos elementos espectrais . . . . . . . . . . . 19

2.3.2 Resolução das Equações de Navier Stokes aplicando o método dos elementos espectrais . . . . . . . . . . . . . 22

2.4 Condições de setup . . . . . . . . . . . . . . . . . . . . . . . . 28

2.4 .1 Condição de Entrada . . . . . . . . . . . . . . . . . . . 28

2.4.2 Condição de Saída . . . . . . . . . . . . . . . . . . . . . . 29

2.4 .3 Condição de Parede . . . . . . . . . . . . . . . . . . . . . . 29

2.4.4 Condição de Parede Invíscida .......... . 29

2.5 Malhas Computacionais . . . . . . . . . . . . . . . . . 29 
2.5.1 Testes de convergência e refino da malha . . . . . . . . . 30

3 Simulações Numéricas $\quad 35$

3.1 Introdução . . . . . . . . . . . . . . . . . . . . 35

3.2 Simulação de Cilindro Isolado . . . . . . . . . . . . . . . . . . 35

3.3 Simulações de Cilindros com Splitter Plate . . . . . . . . . . . . . 38

3.3.1 Simulações com splitter plate posicionado na base do cilindro 39

3.3.2 Simulações com splitter plate com variação do GAP . . . . 42

4 O Método Adjunto $\quad 68$

4.1 Introdução . . . . . . . . . . . . . . . . . . . . 68

4.2 Revisão Bibliográfica . . . . . . . . . . . . . . . . . . . . 68

4.3 Modelagem da Solução Adjunta baseada nas equações de Navier Stokes . . . . . . . . . . . . . . . 76

4.3 .1 Introdução . . . . . . . . . . . . . . . 76

4.3 .2 Equações Adjuntas . . . . . . . . . . . . . . . . . . . 79

4.4 Condições de Contorno Adjuntas . . . . . . . . . . . . . . . . 86

4.4.1 Condições Temporais . . . . . . . . . . . . . . . 87

4.4.2 Formulação das Condições de Contorno . . . . . . . . . . . 88

4.4.3 Considerações finais sobre a Modelagem . . . . . . . . . . 96

5 Resultados da Simulação Adjunta $\quad 99$

5.1 Resultados Preliminares . . . . . . . . . . . . . . . . . . 99

6 Conclusões e Trabalhos Futuros $\quad 104$

$\begin{array}{lr}\text { Referências } & 106\end{array}$

Apêndice A - Forma Generalizada das Equações de Navier Stokes 113

A.1 Introdução . . . . . . . . . . . . . . . . . . . . 113

A.2 A Álgebra Tensorial Generalizada . . . . . . . . . . . . . . 115 
A.3 Equações de Navier Stokes na forma reduzida . . . . . . . . . . 122

A.4 Escoamentos Incompressíveis . . . . . . . . . . . . . . . . . 124

A.5 Conclusões . . . . . . . . . . . . . . . . . . . . 125

$\begin{array}{lr}\text { Apêndice B - Elementos de Área } & 127\end{array}$ 


\section{LISTA DE FiguRAS}

1.1 Modelo de Kármán . . . . . . . . . . . . . . . . . . . . . 5

1.2 Mecanismo de Formação do Vórtice de Gerrard (E), Ilustração da Vorticidade em uma simulação numérica (D) . . . . . . . . . 6

1.3 Configurações de posicionamento do splitter plate . . . . . . . 8

1.4 Variação de $C_{p} b$ em função do GAP . . . . . . . . . . . . . . . . . 11

1.5 Direção das curvas de $C_{p b}$ e $S t$ em função $a / d$ para diversos valores de $G A P \ldots \ldots \ldots \ldots \ldots \ldots . \ldots \ldots \ldots$

1.6 Variação de $S_{t}$ em função do GAP . . . . . . . . . . . . . . . 12

1.7 Visualização do escoamento com splitter plate (KAWAI, 1990) . . . 14

1.8 Contornos de Vorticidade no escoamento de dois cilindros alinhados 15

1.9 Configurações de disposição de splitter plates (ASSI; BEARMAN; KITNEY, 2009) . . . . . . . . . . . . . . . . . . 16

2.1 Regiões do domínio computacional onde serão inseridas as condições de contorno . . . . . . . . . . . . . . . . . . . . . 28

2.2 Malha otimizada . . . . . . . . . . . . . . . 30

3.1 Quadros Temporais ao longo de um período $\mathbf{T}$ do Escoamento ao redor de um cilindro isolado $(R e=140) \ldots \ldots . . . . . .36$

3.2 Evolução do coeficiente de arrasto ao longo do tempo $(R e=140) \quad 37$

3.3 Detalhe na região do escoamento já desenvolvida $(R e=140) \ldots 37$

3.4 Visão Geral da Evolução do coeficiente de sustentação ao longo do tempo $(R e=140) \ldots \ldots \ldots \ldots \ldots$

3.5 Detalhe na região do escoamento já desenvolvida $(R e=140)$. . 38

$3.6 \overline{C_{d}}$ para GAP nulo. . . . . . . . . . . . . . . . . . . . 40

3.7 Amplitude de $C_{l}$ para GAP nulo. . . . . . . . . . . . . . 40

3.8 Quadros temporais da movimentação da vorticidade, $l / d=1,0 . \quad$. 41 
3.9 Valor do Coeficiente médio de arrasto $\overline{C_{d}}$ ao longo do tempo, para $R e=140, l / d=1.0$ e $g / d=0 \ldots \ldots \ldots 41$

3.10 Máxima amplitude de $C_{l}$ ao longo do tempo, para $R e=140, l / d=$ 1.0 e $g / d=0 \ldots \ldots \ldots \ldots . \ldots \ldots 42$

3.11 Coeficiente Médio de Arrasto $\overline{C_{d}}$ versus $g / d$, para $R e=80$ e $l / d=$ $0,5 \ldots \ldots \ldots \ldots \ldots \ldots \ldots \ldots$

3.12 Máxima amplitude de $C_{l}$ versus $g / d$, para $R e=80$ e $l / d=0,5 . \quad$. 43

3.13 Coeficiente Médio de Arrasto $\overline{C_{d}}$ versus $g / d$, para $R e=80$ e $l / d=$ $1,0 \ldots \ldots \ldots \ldots \ldots 4 \ldots \ldots \ldots \ldots$

3.14 Máxima amplitude de $C_{l}$ versus $g / d$, para $R e=80$ e $l / d=1,0 . \quad . \quad 45$

3.15 Coeficiente de Arrasto $\overline{C_{d}}$ versus $g / d$, para $R e=100$ e $l / d=0,5 . \quad 45$

3.16 Máxima amplitude de $C_{l}$ versus $g / d$, para $R e=100$ e $l / d=0,5$. 46

3.17 Coeficiente de Arrasto $\overline{C_{d}}$ versus $g / d$, para $R e=100$ e $l / d=1,0 . \quad 46$

3.18 Máxima amplitude de $C_{l}$ versus $g / d$, para $R e=100$ e $l / d=1,0 . \quad 47$

3.19 Média do Coeficiente de Arrasto $\overline{C_{d}}$ ao longo do tempo, para $R e=$ 100 e $l / d=1,0 \ldots \ldots \ldots \ldots$. . . . . . . . . . . . . 47

3.20 Máxima amplitude de $C_{l}$ ao longo do tempo, para $R e=100 \mathrm{e}$ $l / d=1,0 \ldots \ldots \ldots \ldots \ldots \ldots \ldots \ldots$

3.21 Coeficiente de Arrasto e $\overline{C_{d}}$ versus $g / d$, para $R e=120$ e $l / d=0,5 . \quad 48$

3.22 Máxima amplitude de $C_{l}$ versus $g / d$, para $R e=120$ e $l / d=0,5 . \quad 49$

3.23 Coeficiente de Arrasto $\overline{C_{d}}$ versus $g / d$, para $R e=120$ e $l / d=1,0 . \quad 49$

3.24 Máxima amplitude de $C_{l}$ versus $g / d$, para $R e=120$ e $l / d=1,0 . \quad 50$

3.25 Coeficiente de Arrasto $\overline{C_{d}}$ versus $g / d$, para $R e=140$ e $l / d=0,5 . \quad 51$

3.26 Máxima amplitude de $C_{l}$ versus $g / d$, para $R e=140$ e $l / d=0,5 . \quad 51$

3.27 Coeficiente de Arrasto $\overline{C_{d}}$ versus $g / d$, para $R e=140$ e $l / d=1,0 . \quad 52$

3.28 Máxima amplitude de $C_{l}$ versus $g / d$, para $R e=140$ e $l / d=1,0 . \quad 52$

3.29 Quadros temporais da movimentação da vorticidade, $a / d=1,0$, $g / d=3,4 \ldots \ldots \ldots \ldots \ldots \ldots \ldots$

3.30 Quadros temporais da movimentação da vorticidade, $l / d=1,0$, $g / d=4,0 \ldots \ldots \ldots \ldots \ldots \ldots \ldots$ 
3.31 St $\times g / d$, para $l / d=0,5$ e $R e=80$.

$3.32 S t \times g / d$, para $l / d=0,5$ e $R e=100$.

$3.33 S t \times g / d$, para $l / d=0,5$ e $R e=120$. 56

$3.34 S t \times g / d$, para $l / d=0,5$ e $R e=140$. 56

$3.35 S t \times g / d$, para $l / d=1,0$ e $R e=80$. 57

$3.36 S t \times g / d$, para $l / d=1,0$ e $R e=100$. 57

$3.37 S t \times g / d$, para $l / d=1,0$ e $R e=120$. 57

$3.38 S t \times g / d$, para $l / d=1,0$ e $R e=140$. 58

$3.39 l_{f} \times g / d$, para $l / d=0,5$ e $R e=80$. 59

$3.40 l_{f} \times g / d$, para $l / d=0,5$ e $R e=100$. 59

$3.41 l_{f} \times g / d$, para $l / d=0,5$ e $R e=120$. 59

$3.42 l_{f} \times g / d$, para $l / d=0,5$ e $R e=140$. 60

$3.43 l_{f} \times g / d$, para $l / d=1,0$ e $R e=80$. 60

$3.44 l_{f} \times g / d$, para $l / d=1,0$ e $R e=100$.

$3.45 l_{f} \times g / d$, para $l / d=1,0$ e $R e=120$.

$3.46 l_{f} \times g / d$, para $l / d=1,0$ e $R e=140$

$3.47 \overline{-C_{p b}} \times g / d$, para $l / d=0,5$ e $R e=80$.

$3.48 \overline{-C_{p b}} \times g / d$, para $l / d=0,5$ e $R e=100$.

$3.49 \overline{-C_{p b}} \times g / d$, para $l / d=0,5$ e $R e=120$.

$3.50 \overline{-C_{p b}} \times g / d$, para $l / d=0,5$ e $R e=140$.

$3.51 \overline{-C_{p b}} \times g / d$, para $l / d=1,0$ e $R e=80$.

$3.52 \overline{-C_{p b}} \times g / d$, para $l / d=1,0$ e $R e=100$.

$3.53 \overline{-C_{p b}} \times g / d$, para $l / d=1,0$ e $R e=120$.

$3.54 \overline{-C_{p b}} \times g / d$, para $l / d=1,0$ e $R e=140$.

3.55 Distribuição de pressão ao longo do cilindro 66

5.1 Condição de contorno de saída na solução adjunta utilizando o grau de precisão do polinômio interpolador igual a 25 . 
5.2 Condição de contorno de saída na solução adjunta utilizando o grau de precisão do polinômio interpolador igual a 50 . . . . . . . 102

5.3 Escoamento adjunto ao redor do cilindro, $\mathrm{Re}=30 \ldots \ldots$

A.1 Espaço Físico e Tranformado . . . . . . . . . . . . . . . . . . . . . 114 


\section{LISTA DE TABELAS}

2.1 Coeficientes dos algoritmos das família Adams-Bashforth . . . . . 25

2.2 Coeficientes dos algoritmos das família Adams-Moulton . . . . . . 25

2.3 Testes de convergência para grau polinomial . . . . . . . . . . . 31

2.4 Testes de convergência para a ordem de integração temporal . . 32

2.5 Testes de convergência para o avanço no tempo adimensional . . . 32

2.6 Testes de convergência para largura da malha . . . . . . . . . 33

2.7 Testes de convergência para largura da malha . . . . . . . . . 33

2.8 Comparação dos Resultados entre diversas referências . . . . . . . 34

3.1 Simulações Realizadas com Splitter Plate - GAP Nulo . . . . . . 38

3.2 Simulações Realizadas com Splitter Plate - GAP Variável . . . . . 39

3.3 Resumo dos resultados - Média Temporal do Coeficiente de Arrasto 54

3.4 Resumo dos resultados - Amplitude do Coeficiente de Sustentação 54

3.5 Resumo dos resultados - Número de Strouhal . . . . . . . . . . . . 58

3.6 Resumo dos resultados - Comprimento de Formação . . . . . . . . 62

3.7 Resumo dos resultados - Coeficiente de Pressão na Base do Cilindro 67

4.1 Condições de Contorno . . . . . . . . . . . . . . . . . . . . . . . 97 


\section{ACRÔNIMOS}

$\begin{array}{ll}\text { CFD } & \text { Computational Fluid Dynamics } \\ \text { CFL } & \text { Número de Courant-Friedrichs-Lewy } \\ \text { NDF } & \text { Núcleo de Dinâmica dos Fluidos } \\ \text { VIV } & \text { Vibração induzida por Vórtices (Vortex Induced Vibration) } \\ \text { RMS } & \text { Valor Quadrático Médio (Root mean Square) } \\ \text { EPUSP } & \text { Escola Politécnica da Universidade de São Paulo } \\ \text { PME } & \text { Departamento de Engenharia Mecânica - EPUSP } \\ \text { SEM } & \text { Método dos Elementos Espectrais } \\ \text { FEM } & \text { Método dos Elementos Finitos }\end{array}$




\section{INTRODUÇÃO}

A dinâmica dos fluídos computacional (CFD) assumiu uma posição importante no desenvolvimento de projetos relacionados a mecânica dos fluídos, cuja base teórica envolve modelos baseados em conservação de massa, quantidade de movimento e de energia.

Ao longo da história, esses princípios foram modelados através de equações diferenciais não-lineares, para as quais não se conhecem soluções análiticas gerais. As exceções ficam restritas a apenas alguns problemas com significativas hipóteses simplificadoras. Tais condições favoreceram o desenvolvimento de técnicas de simulação numérica, como alternativa natural aos métodos analíticos, para a obtenção de soluções aproximadas.

Com o advento dos modernos computadores, que a cada ano aumentam sua velocidade de processamento, os métodos numéricos ganharam ainda mais espaço com soluções mais robustas, proporcionando ao engenheiro respostas mais imediatas de um determinado estudo em questão. Com esta facilidade, já existem inúmeras aplicações que se utilizam do $\mathrm{CFD}$, como em projetos de aerodinâmica e hidrodinâmica, sistemas de refrigeração e até mesmo dentro da ciência médicahospitalar.

Dentro desta variedade de temas de relevância em CFD, duas questões concernem o presente trabalho: o escoamento em torno de corpos rombudos (mais precisamente cilindros), amplamente estudado na comunidade científica (MENEGHINI, 1993; GIORIA, 2009; CARMO, 2005) e a aplicação de métodos de otimização em problemas de engenharia, voltados para a busca de extremos de uma determinada função ou método de cálculo (VOLPE, 2004; VOLPE, 2006; CHIEREGATTI, 2008; CEZE, 2008; HAYASHI, 2009).

Os longos e flexíveis tubos de extração de petróleo, conhecidos como risers são essenciais para a extração deste recurso em águas profundas, sendo essa atividade a principal fonte de petróleo no Brasil. Esses elementos estruturais flexíveis oscilam quando imersos em correntes marítimas, devido a formação e despren- 
dimento alternado de vórtices (vortex shedding), que proporciona variações nas forças de pressão no sentido transversal do escoamento.

Assim, o entendimento deste problema se faz necessário na busca de soluções que possam minimizar este tipo de fenômeno, e para isso, é importante uma modelagem para este tipo de escoamento, sendo o cilindro de seção circular uma geometria relevante nessas aplicações.

Muitos institutos de pesquisa ao redor do mundo dedicam-se ao desenvolvimento de pesquisa para o controle do escoamento sobre corpos rombudos. Há inúmeros temas que são estudados, tais como vibrações forçadas (MENEGHINI, 1993; MENEGHINI; BEARMAN, 1995; MENEGHINI, 2002; BEARMAN; CURRIE, 1979; FENG, 1968) e dispositivos acoplados aos cilindros como tip-wires, vortex generators e splitter plates.

Com a experiência adquirida nos métodos de otimização desenvolvidos em trabalhos anteriores somados a vasta bibliografia existente em relação a escoamento ao redor de cilindros, esse trabalho buscará iniciar a integração de um método de otimização conhecido como método adjunto com o estudo de um problema relacionado a este tipo de escoamento, que é o estudo do posicionamento de splitter plates em cilindros de seção circular.

\section{$1.1 \quad$ Objetivos}

Para desenvolvimento e aplicação de um método de otimização como o adjunto, são necessárias algumas etapas preliminares até que o método possa ser validado e seu uso em alguma aplicação prática possa ser feito.

O presente trabalho terá como objetivo principal essas etapas preliminares que serão alicerce para o desenvolvimento desta pesquisa como um todo. As simulações do método adjunto em si não serão escopo deste trabalho pois se trata de uma etapa posterior ao que foi produzido nesta dissertação. Apenas como complemento, serão apresentados os testes preliminares das primeiras simulações adjuntas de maneira a mostrar ao leitor os próximos passos que serão realizados.

A primeira etapa foi a escolha de um problema simples que pudesse ser resolvido por outros métodos de otimização, ou até que fosse possível uma varredura completa de uma faixa do universo de soluções, para que na etapa de validação do método adjunto, esse casos sejam base de comparação dos resultados encontrados.

Assim, foi escolhido o problema de controle da esteira de vórtices de um 
cilindro de seção circular, utilizando o splitter plate. Além de sua simplicidade, já que o splitter plate é apenas uma placa plana, o problema escolhido possui relevância na comunidade científica, pois a placa possui grande efetividade no controle das forças atuantes no cilindro.

Com o problema escolhido, toda fundamentação teórica deste tipo de escoamento foi estudada, com o objetivo de se entender a física envolvida e através de uma vasta revisão bibliográfica, verificar quais eram as principais variáveis avaliadas, escolhendo uma como função objetivo do problema, ou seja, a medida de mérito que se usaria para otimizar a geometria do splitter plate.

A etapa seguinte foi uma análise sistemática de diversos escoamentos de cilindros com splitter plate, variando seu comprimento e sua distância em relação ao corpo rombudo. Como esta análise necessitou de muitas simulações numéricas, houve uma preocupação com a geometria e o refinamento da malha computacional, além dos parâmetros da simulação que foram escolhidos para se garantir uma convergência satisfatória, num intervalo de tempo razoável.

Os resultados dessas simulações foram comparados com as simulações do cilindro isolado, para se quantificar a melhoria efetiva de uma certa propriedade. Com os resultados, foi possível verificar quais configurações chegavam ao extremos da medida de mérito.

Paralelamente o problema adjunto foi analisado matematicamente utilizando as equações de Navier Stokes como restrição do problema. A partir da medida de mérito escolhida, a formulação do gradiente de sensibilidade foi obtida e as equações ajuntas foram encontradas, desenvolvendo a seguir as devidas condições de contorno para resolução do problema.

A abordagem dessa dissertação para o problema limitará-se à simulações bidimensionais devido a restrições de tempo para execução do trabalho. Porém, as metodologias apresentadas podem ser extendidas a outras funções de mérito, bem como para simulações tridimensionais.

Ressaltando novamente o conteúdo acima, a presente dissertação objetivou criar os subsídios necessários para que a pesquisa sobre a aplicação do método adjunto neste tipo de escoamento pudesse dar um novo passo em seu desenvolvimento, deixando um produto final que alimentará os trabalhos futuros com material necessário para validação dos resultados das simulações adjuntas. 


\subsection{Escoamento ao redor de cilindros}

O escoamento ao redor de um cilindro isolado é uma linha de pesquisa que recebe grande atenção da comunidade científica, resultando em um grande número de publicações sobre o assunto.

Segundo Bearman (1965) apud Meneghini (2002), um corpo rombudo é definido como um corpo imerso em uma corrente de fluido gerando separação do escoamento em uma grande parcela de sua superfície. Essa separação provoca a formação de duas camadas cisalhantes na região imediatamente posterior ao corpo. Estas camadas possuem sentidos de vorticidade opostos constituindo um sistema instável.

A interação dessas camadas cisalhantes é a principal responsável pela formação e desprendimento de vórtices. Tal fenômeno pode causar o que conhecemos como vibração induzida por vórtices (em inglês Vortex Induced Vibration) cuja sigla é VIV.

A primeira investigação científica do problema aconteceu em 1878 feita por Strouhal (RAYLEIGH, 1896). Ele estudou a vibração de fios imersos em uma corrente de ar e com os resultados concluiu que a frequência do som emitida $\left(f_{s}\right)$ resultante do movimento do fio era dependente apenas do seu diâmetro $(d)$ e a velocidade $\left(U_{\infty}\right)$ relativa do fluido em relação ao fio. Outra descoberta foi quando a frequência do som atingia valores de frequência natural do fio, este som se itensificava. A relação encontrada foi a seguinte:

$$
f_{s}=\frac{0.185 U}{D}
$$

Em 1879, o próprio Lord Rayleigh descobriu que a oscilação ocorria na direção transversal do escoamento sendo que anteriormente se achava que a mesma ocorria na direção da corrente. Com isso, Lord Rayleigh homenageou o precursor dos estudos, definindo assim o número de Strouhal apresentado nas equações 1.2. Com essa definição, conclui-se que Strouhal estudou um fenômeno cujo adimensional relacionado ao diâmetro do fio era de 0,185.

O primeiro modelo da esteira de vórtices foi idealizado por Karman (1911) e é descrito em Meneghini (2002). A fileira de vórtices foi idealizada no caso de um fluído invíscido considerando cada vórtice com circulação concentrada ao longo de uma linha perpendicular ao plano do escoamento. Suas análises foram feitas considerando a ausência do corpo rombudo pois sua preocupação estava voltada 
para a estabilidade da esteira e uma maneira de representá-la através de uma distribuição ideal de vórtices (MENEGHINI, 2002). A figura 1.1 ilustra o modelo de Kármán sendo as variáveis $a_{w}$ e $h_{w}$ definidas através de equações presentes Meneghini (2002) e são determinadas através de definições de estabilidade.

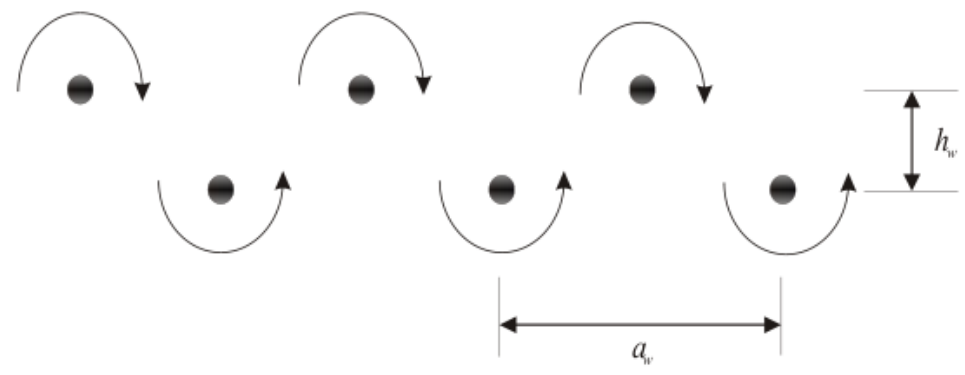

Figura 1.1: Ilustração do modelo de Kármán para uma dupla fileira de vórtices (MENEGHINI, 2002)

A teoria do escoamento potencial (fluídos ideais não viscosos) estudada por Karman (1911) diz que só há duas formas de um corpo sofrer ação de forças do escoamento: Caso exista aceleração do corpo, onde o mesmo receberá forças de reação do fluído, e em caso de velocidade constante, a única forma de existir uma força é no caso da circulação $\Gamma$ ser diferente de zero.

De posse desse conhecimento, chegou-se a uma séria discordância no modelo de fluido ideal. A teoria do escoamento potencial aplicada ao redor de um corpo simétrico, que se move a uma velocidade constante, fornecia uma força de arrasto nula. Todavia, por experiência dos pesquisadores, era sabido que existia uma força de arrasto com certa magnitude e durante quase dois séculos buscaram a resposta para essa questão conhecida como Paradoxo de D'Alembert (MENEGHINI, 2002).

Somente no século XX, Prandtl propôs em sua teoria que existia uma região próxima a superfície do corpo onde as forças viscosas eram significativas e que fora desta região, os efeitos viscosos permaneciam irrelevantes, sendo bem modeladas pela teoria potencial. Assim, reconheceu-se que o Paradoxo de D'Alembert só existia pois os pesquisadores não consideravam a camada limite em seus modelos.

O próximo grande avanço na modelagem deste tipo de problema foi conquistado por Gerrard (1966) que investigou profundamente os fenômenos físicos envolvendo a formação de vórtices a jusante dos corpos rombudos. Seu foco foi diferente de Karman (1911), onde ao invés de estudar os conceitos de estabilidade 
citados anteriormente, apresentou seu modelo como uma interação mútua entre as duas camadas cisalhantes que surgiam dos dois lados do cilindro. A formação do vórtice era composta de 3 partes que estão representadas na figura 1.7 juntamente com um resultado do modelo numérico mostrando o resultado semelhante àqueles obtidos no modelo.
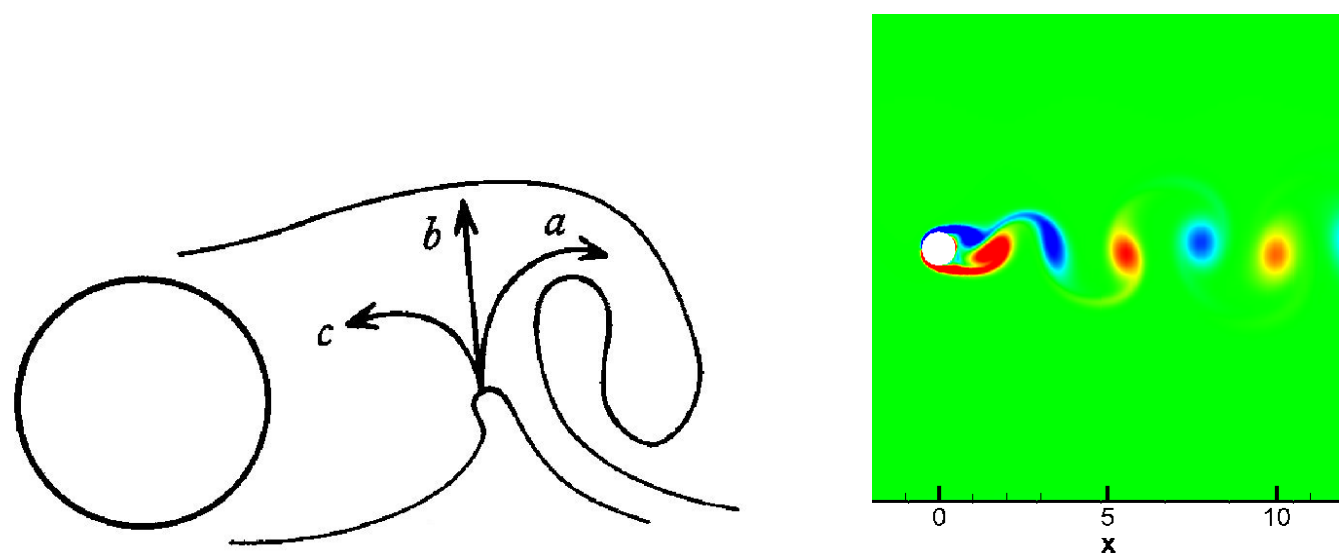

Figura 1.2: Esquerda, mecanismo de formação de vórtices atrás do cilindro. Reprodução de (GERRARD, 1966). Direita, simulação numérica do mesmo problema, com destaque para a visualização da formação dos vórtices $(R e=140)$.

Como observado em Meneghini (2002), a vorticidade das camadas cisalhantes tem sinais opostos, e sob essa condição, as linhas de corrente das camadas cisalhantes acabam deslocando-se de acordo com as direções propostas por Gerrard (1966). A primeira parcela dessas linhas (a) movimenta-se no mesmo sentido da camada cisalhante oposta, alimentando o vórtice em fase final de formação; a segunda parcela (b) será responsável pelo "corte" da camada cisalhante oposta, terminando assim a formação do vórtice imediatamente antes ao que está sendo formado; e finalmente a última parcela (c) ocupa o espaço deixado pela camada oposta deslocando-se no sentido oposto ao vórtice anterior e sendo alimentado pela parcela (a) da camada cisalhante oposta, reiniciando o processo.

Em Meneghini (2002), o autor divide o escoamento ao redor do corpo rombudo em três áreas: a primeira sendo interior a camada limite, onde as forças viscosas são relevantes, a segunda, sendo a região do escoamento onde as forças viscosas são desprezíveis, podendo ser modelada pela teoria do escoamento potencial e a terceira, sendo a região da esteira de vórtices, a jusante do corpo rombudo.

De maneira a introduzir os parâmetros que serão analisados neste trabalho, serão definidos a seguir os coeficientes e adimensionais utilizados para a análise dos resultados. Para descrição do escoamento, o adimensional mais importante será o número de Reynolds $(R e)$. Em relação a oscilação do corpo, um parâmetro 
de avaliação será a sua frequência, caracterizada pelo número de Strouhal $(S t)$. Finalmente para mensuração das forças, os coeficientes calculados serão o de arrasto $\left(C_{d}\right)$, sustentação $\left(C_{l}\right)$ e o coeficiente de pressão da base do cilindro $\left(C_{p b}\right)$ :

$$
\left\{\begin{array} { l } 
{ R e = \frac { U _ { \infty } d } { \nu } } \\
{ S t = \frac { f d } { U } } \\
{ q _ { \infty } = \frac { 1 } { 2 } \rho _ { \infty } U _ { \infty } ^ { 2 } }
\end{array} \quad \left\{\begin{array}{l}
C_{p}=\frac{p-p_{\infty}}{q_{\infty}} \\
C_{l}=\frac{\|\mathbf{L}\|}{q_{\infty} d} \\
C_{d}=\frac{\|\mathbf{D}\|}{q_{\infty} d}
\end{array}\right.\right.
$$

É importante ressaltar que os coeficientes de arrasto e sustentação são calculados por unidade de comprimento. Adicionalmente, $\nu$ é a viscosidade cinemática, $\rho_{\infty}$ and $U_{\infty}$ são a massa específica e velocidade ao longe, respectivamente. O parâmetro $d$ simboliza o comprimento de referência, no caso o diâmetro do cilindro e $f$ a frequência de oscilação. Adicionamente temos $D$ e $L$ como sendo as forças de arrasto e sustentação respectivamente.

\subsection{O Splitter Plate}

Por sua facilidade de montagem e operação, o splitter plate torna-se um importante dispositivo de controle da esteira de vórtices no escoamento ao redor de corpos rombudos. O seu princípio físico é muito simples, pois sua presença causa um aumento da distância (em relação ao cilindro) de formação da esteira $\left(l_{f}\right)$, sendo esse o principal fator de indução de oscilação no cilindro, ocasionado uma redução significativa no coeficiente de arrasto médio $C_{d}$ bem como a amplitude da própria oscilação.

Um dos principais estudos sobre escoamentos ao redor de cilindros com splitter plate foi feito por Igarashi (1982) que classificou os mesmos de acordo com a maneira que era montado ao corpo rombudo. Há duas configurações possíveis, a primeira, os splitter plates são presos a base do cilindro, e a segunda opção são as placas montadas de tal maneira que exista um vão $(G A P)$ entre a base do cilindro e o bordo de ataque do splitter plate (figura 1.3).

\subsection{Revisão Bibliográfica}

Nesta seção, serão discutidas as principais referências utilizadas no estudo de escoamento de cilindros com splitter plates que aprofundaram o conhecimento e proporcionaram o desenvolvimento deste trabalho. Buscou-se organizar cro- 


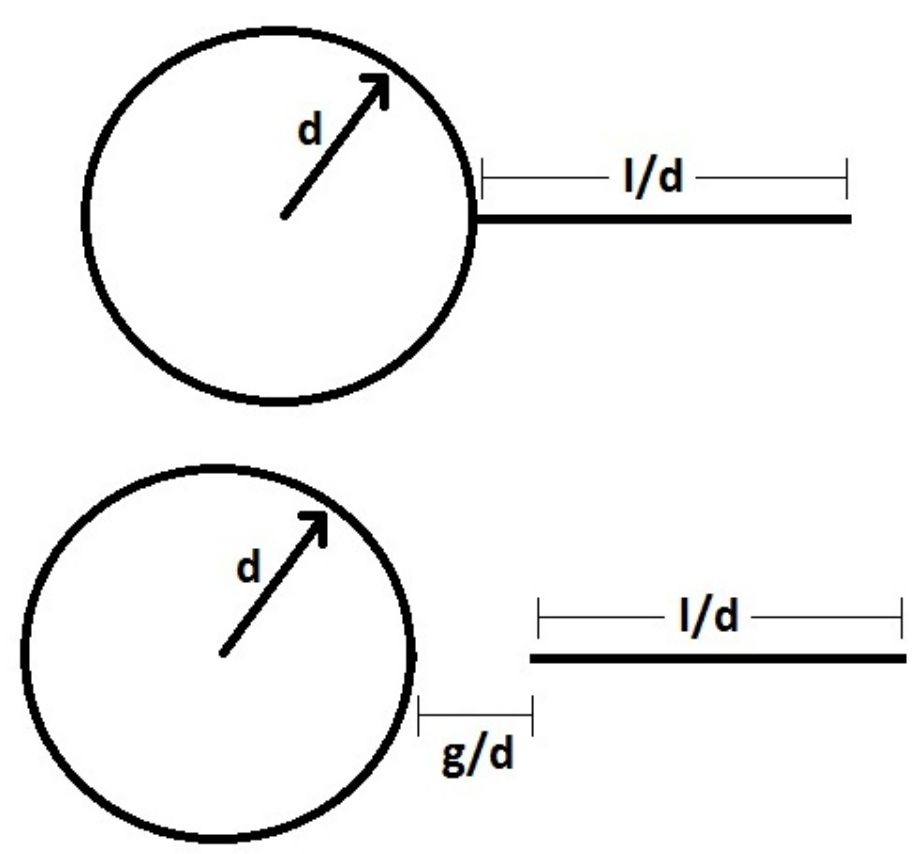

Figura 1.3: Configurações de posicionamento do splitter plate, Acima, GAP nulo; Abaixo, GAP variável

nologicamente os trabalhos, porém quando necessário, serão adicionados fatos relevantes que complementem a apreciação do trabalho pelo leitor.

O primeiro registro de trabalhos com splitter plates foi feito por Roshko (1954a), Roshko (1954b) que realizou estudos semi-empíricos em escoamentos ao redor de corpos rombudos. No estudo foram analisados cilindros com splitter plates, variando em configurações com e sem GAP entre as superfícies para o valor de $R e=1,45 \times 10^{4}$. O autor observou dois resultados importantes: 1-) A presença da placa afetava a distribuição de pressão $\left(C_{p}\right)$ em toda a superfície do cilindro, e não somente na região de separação do escoamento; 2-) O valor do número de Strouhal $(S t)$ foi diminuindo conforme o splitter plate foi se afastando do cilindro chegando até um valor crítico onde se caracterizava o menor valor do adimensional. Após esse ponto crítico, o adimensional retornava ao valor de um cilindro sem splitter plate. O coeficiente de pressão na base do cilindro $\left(C_{p b}\right)$ assumiu o mesmo comportamento, com a diminuição de seu módulo até o ponto crítico e após esse ponto, um aumento brusco para valores próximos de uma situação de escoamento apenas com o cilindro.

O resultado de Roshko foi a primeira evidência da presença de um ponto ótimo de diminuição do arrasto e da frequência de vibração utilizando o splitter plate. A conclusão na referência foi que enquanto o splitter plate estava em uma posição abaixo do valor crítico, ele induzia as camadas cisalhantes a interagirem 
após seu bordo de fuga, afastando tal interação do cilindro. Esse afastamento diminuia a influência dos vórtices sobre o corpo rombudo, diminuindo assim os efeitos de VIV. Após a passagem desse ponto crítico, as camadas cisalhantes voltam sua interação nas próximidades do cilindro, mais precisamente entre a base do cilindro e o bordo de ataque splitter plate. Portanto, a presença do splitter plate tornava-se irrelevante nessa situação.

Em 1965, Bearman (1965) realizou estudos com escoamento em corpos com o bordo de fuga chanfrado e também utilizando splitter plates. A faixa de Reynolds da análise foi de $1,4 \times 10^{5} \leq R e \leq 2,56 \times 10^{5}$. De posse de muitos resultados, sua principal conclusão foi que o coeficiente de pressão na base do cilindro $C_{p}$ era inversamente proporcional ao que ele definiu como comprimento de formação dos vórtices (em inglês, formation length, $l_{f}$ ). Esse comprimento era definido como a distância entre o ponto de encontro das camadas cisalhantes utilizando a base do cilindro como referência. Para obtenção dessa distância, procurava-se o ponto no eixo do escoamento onde o valor quadrático médio (RMS) da velocidade fosse mínimo.

A partir destes resultados, formulou-se a teoria de que a proporcionalidade encontrada por Bearman (1965) era válida nas proximidades do cilindro até o ponto crítico encontrado por Roshko alguns anos antes. Esse conceito de comprimento de formação é utilizado até os dias de hoje como forma de análise do comportamento do escoamento.

É importante ressaltar que no modelo de Gerrard (1966), o comprimento de formação $l_{f}$ é facilmente localizado pois será o ponto onde a camada cisalhante cruza o eixo do escoamento $(\mathrm{x})$ e ocupa o espaço deixado pela camada oposta. $\mathrm{O}$ autor realizou experimentos com cilindros com splitter plates com GAP na faixa de Reynolds de $R e=2 \times 10^{4}$ reproduzindo os experimentos de Roshko (1954a) e assim pôde entender claramente como o splitter plate atuava sobre o escoamento.

A partir do modelo apresentado na figura 1.7 concluiu-se que o splitter plate impedia a movimentação da camada cisalhante para o espaço da camada oposta, provocando assim um movimento dessas camadas paralelamente a placa, ou seja, afastando-se do cilindro. O fenômeno descrito na figura 1.7 só ocorria após o bordo de fuga do splitter plate. No caso da passagem do ponto crítico para splitter plates com $G A P$, o espaço entre o cilindro e o bordo de ataque da placa era suficiente para a camada cisalhante executar seu movimento de avanço sobre a camada oposta e assim eliminar os benefícios do dispositivo, reduzindo novamente o comprimento de formação para o valor de um cilindro sem splitter plate. 
Uma configuração que não foi analisada na referência acima foi o splitter plate fixado na base do cilindro, ou seja, sem GAP. Em Apelt, West e Szewczyk (1973), os autores executaram uma série de experimentos apenas com splitter plates fixados na base do cilindro na faixa de Reynolds de $10^{4} \leq R e \leq 5 \times 10^{4}$. Foi determinada uma classificação dos splitter plates quanto ao seu comprimento sendo considerados curtos quando $l / d \leq 1$ e longos quando ultrapassam esse valor.

Eles concluiram que splitter plates curtos diminuiam a largura da camada cisalhante e estabilizavam o ponto de separação da camada limite no cilindro. Entretanto por possuir um comprimento curto, apesar das camadas cisalhantes interagirem no bordo de fuga da placa, o comprimento de formação permanecia baixo, sem grandes benefícios de redução de arrasto. Por outro lado, splitter plates longos conseguiam tranferir a interação das camadas cisalhantes para comprimentos de formação maiores, todavia pelo fato de possuir um longo comprimento, o arrasto da própria placa torna-se significativo, sendo uma limitação do benefício.

Em 1982, o trabalho experimental presente em Igarashi (1982) buscou reproduzir todos os trabalhos dos autores acima, utilizando os resultados dos mesmos como parâmetro de comparação. Ele realizou diversos experimentos em cilindros com splitter plates, nas configurações de GAP nulo e variável e para diversos comprimentos de placa. Seu intevalo de trabalho para o comprimento do splitter plate e do $G A P$ ficou em $0,29 \leq l / d \leq 1,76$ e $0 \leq g / d \leq 4$, respectivamente, enquanto que para o número de Reynolds ficou em $1.3 \times 10^{4} \leq R e \leq 5.8 \times 10^{4}$.

Em sua revisão bibliográfica, o autor dividiu os trabalhos em três grandes frentes: a primeira, focada na frequência de vibração do cilindro e seu estudo através do número de Strouhal, sendo que os trabalhos inseridos nessa categoria foram os de Roshko e Gerrard (ROSHKO, 1954a; ROSHKO, 1954b; GERRARD, 1966); a segunda frente focava na influência do splitter plate no comportamento da camada cisalhante e de seus efeitos nos coeficientes de arrasto e sustentação, com inclusão dos trabalhos de Bearman e Apelt neste grupo (BEARMAN, 1965; APELT; WEST; SZEWCZYK, 1973); e finalmente a terceira frente, focada nos fenômenos de transferência de calor na região de separação da camada turbulenta atrás do cilindro, sendo esse um trabalho que o autor realizou anteriormente (IGARASHI, 1984) motivado pelo estudo de trocadores de calor do tipo "banco de tubos".

Os resultados mostraram variações na frequência de vibração, através do número de Strouhal $(S t)$, do coeficiente de pressão médio na base do cilindro $\left(\overline{C_{p b}}\right)$ 
e arrasto médio para diversos comprimentos de placa e de GAP.

Para $\overline{C_{p b}}$, o autor concluiu que em configurações de $G A P$ variável, quanto maior o comprimento do splitter plate, maior seria o valor do ponto crítico, ou seja, mais afastado a camada cisalhante começava a interagir. A figura 1.4, reproduzida da própria referência, ilustra os diversos resultados encontrados:

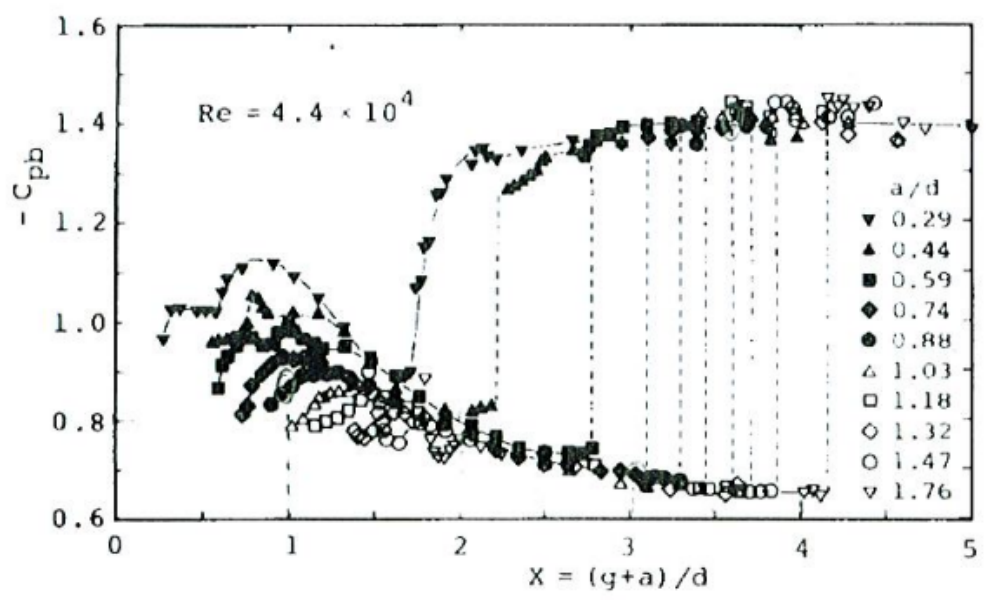

Figura 1.4: Variação de $C_{p} b$ em função do GAP, para diversos comprimentos de placa. Reprodução de (IGARASHI, 1982)

Um ponto a ser observado é que este benefício limitava-se apenas até comprimentos $l / d \leq 1,76$ e que acima deste valor, a posição do ponto crítico permanecia praticamente constante. Adicionalmente, Igarashi também observou que para pequenos valores de $G A P(g / d \leq 0,4)$, o comportamento de $C_{p b}$ não seguia decrescente, para quaisquer valores de $l / d$, sendo que este resultado não tinha sido mencionado em nenhum dos trabalhos anteriores. A figura 1.5 é uma reprodução da descrição do comportamento dos valores de $C_{p b}$ e $S_{t}$ para os comprimentos de splitter plates em (IGARASHI, 1982)

Foram definidas pelo autor diversas regiões $\left(A_{1}, A_{2}, A_{3}, B\right.$ e $\left.C\right)$ sendo separadas por valores intermediários de $\operatorname{GAP}\left(G_{v}, G_{p}\right.$ e $G_{c}$, sendo o último o valor crítico). Na faixa de valores de $0,29 \leq l / d \leq 0,59$, o valor de $G_{p}$ é inversamente proporcional ao comprimento do splitter plate, para $0,74 \leq l / d \leq 1,18, G_{p}$ se torna praticamente constante e para comprimentos maiores, ele decresce a partir do valor do intervalo anterior. No caso de $G_{v}$, ele é praticamente constante na faixa em que ele ocorre.

No caso do número de Strouhal $(S t)$, a figura 1.6 apresenta a variação do adimensional. Na faixa de $0,29 \leq l / d \leq 0,88$, o valor de $S t$ é próximo do valor de um cilindro imerso no escoamento sem splitter plate. Na medida em que o valor do $G A P$ vai aumentando, o valor de $S t$ cresce no início até seu valor máximo 


\begin{tabular}{|c|c|c|c|c|}
\hline \multicolumn{5}{|c|}{ Tipo $1: 0.29 \leq a / d \leq 0.88$} \\
\hline Pontos & \multicolumn{2}{|c|}{$\mathrm{A}_{1}$} & B & $\mathrm{C}$ \\
\hline G & 0 & Gp & Gc & \\
\hline \multicolumn{5}{|l|}{$-C_{p b}$} \\
\hline \multicolumn{5}{|l|}{ St } \\
\hline \multicolumn{5}{|c|}{ Tipo $2: 1.03 \leq a / d \leq 1.76$} \\
\hline Pontos & $A_{2}$ & $A_{3}$ & B & $\mathrm{C}$ \\
\hline G & 0 & $\mathrm{Gp}$ & Gc & \\
\hline$-C_{p b}$ & $\ldots$ & & & \\
\hline St & & & & \\
\hline & & & & \\
\hline
\end{tabular}

Figura 1.5: [Direção das curvas de $C_{p b}$ e $S t$ em função $a / d$ para diversos valores de GAP. Reproduzido de Igarashi (1982)

e logo após inicia uma queda abrupta até o valor de $G_{p}$ como pode ser visto esquematicamente na figura 1.5. Após o valor de $G_{p}$, a diminuição de $S t$ é muito suave até se chegar a $G_{c}$, onde ocorre a descontinuidade e a volta para o valor do cilindro isolado. Nos casos de splitter plates maiores $(1,03 \leq l / d \leq 1,76)$, é possível ainda alguma redução em $S t$ até a descontinuidade. Na figura 1.5 se observa o comportamento do número de Strouhal em comparação a $C_{p b}$.

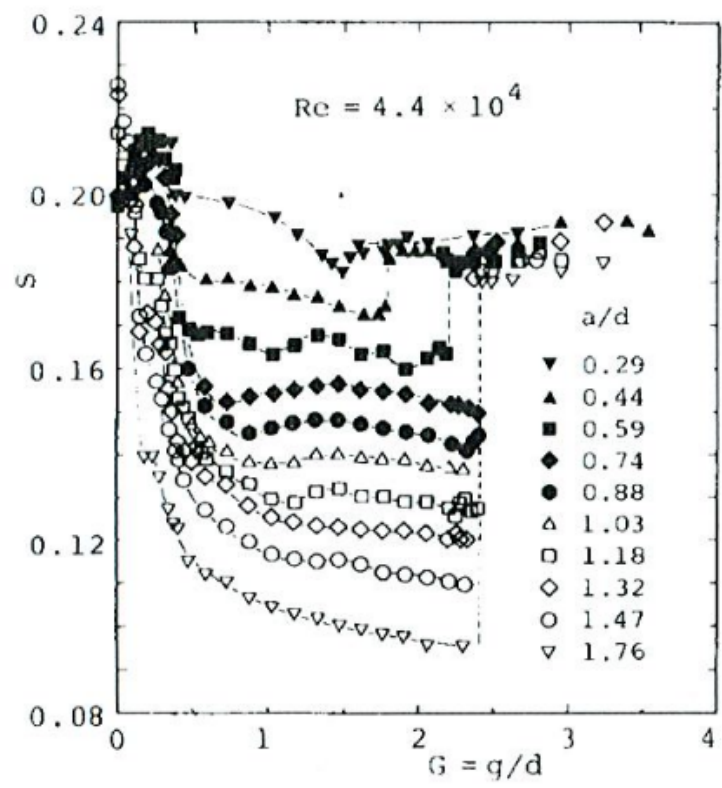

Figura 1.6: Variação de $S_{t}$ em função do GAP, para diversos comprimentos de placa. Reprodução de (IGARASHI, 1982)

O autor também investigou como a variação do número de Reynolds poderia interferir em $C_{p b}$, St e também no comprimento de formação $l_{f}$. Os resultados mostraram que na região C (figura 1.5) próximo a $G_{p}$, conforme o aumento de $R e$, 
o número de Strouhal e o coeficiente de pressão na base do cilindro diminuiam suavemente, enquanto nas outras regiões, abaixo do comprimento crítico, não houve variações nestes parâmetros na faixa de $1,3 \times 10^{4} \leq R e \leq 4,4 \times 10^{4}$. Os valores de $G_{v}, G_{p}$ e $G_{c}$ tiveram a tendência de crescer lentamente com o aumento de Re. Os efeitos encontrados foram considerados insignificantes por Igarashi para o problema.

As principais conclusões que o autor apresentou no trabalho foram a independência dos coeficientes em relação ao número de Reynolds, a descontinuidade após o comprimento crítico teve o mesmo comportamento de um escoamento de dois cilindros dispostos em "tandem" e foi possivel mapear o comportamento dos coeficientes ao longo das variações de GAP.

No final dos anos 80, Unal e Rockwell (1988a), Unal e Rockwell (1988b) publicaram novos experimentos de escoamentos com splitter plates. O primeiro trabalho analisou as instabilidades hidrodinâmicas que apareciam no escoamento ao redor do cilindro em baixos número de Reynolds ( $440 \leq R e \leq 5040)$, enquanto o segundo analisou splitter plates muito longos $(l / d=24)$ em vários valores de GAP $(0 \leq l / d \leq 15)$. Os resultados mostraram novamente que o splitter plate causa modificações no comportamento da esteira de vórtices, porém foi descoberto que o mesmo não era capaz de inibir a instabilidade das camadas cisalhantes.

Já na década de 90, Kawai (1990) realizou simulações numéricas do escoamento ao redor de cilindros com splitter plates para vários comprimentos e valores de GAP. Foi utilizado o método dos vórtices discretos e o modelo representou o comportamento do escomento visto nos ensaios experimentais do passado. Entretanto, os valores númericos foram muito diferentes aqueles encontrados anteriormente, com destaque ao valor do GAP crítico que tinha, em média, metade do valor encontrado nos trabalhos de diversos autores. A justificativa para as diferenças encontradas foi que os valores do número de Reynolds utilizados são bem abaixo das referências anteriores. Este resultado mostrou que a independência em relação ao número de Reynolds pode ser válida apenas em valores elevados do adimensional e que os resultados das simulações numéricas poderiam também apresentar comprimentos críticos abaixo dos valores encontrados em experimentos.

Em trabalhos mais recentes foram apresentados novas simulações numéricas para escoamentos com baixo número de Reynolds. Um deles (KWON; CHOI, 1996), realizou simulações na faixa de $80 \leq R e \leq 160 \mathrm{com}$ splitter plates presos ao cilindro, ou seja, com GAP nulo. O foco principal do trabalho estava na variação 


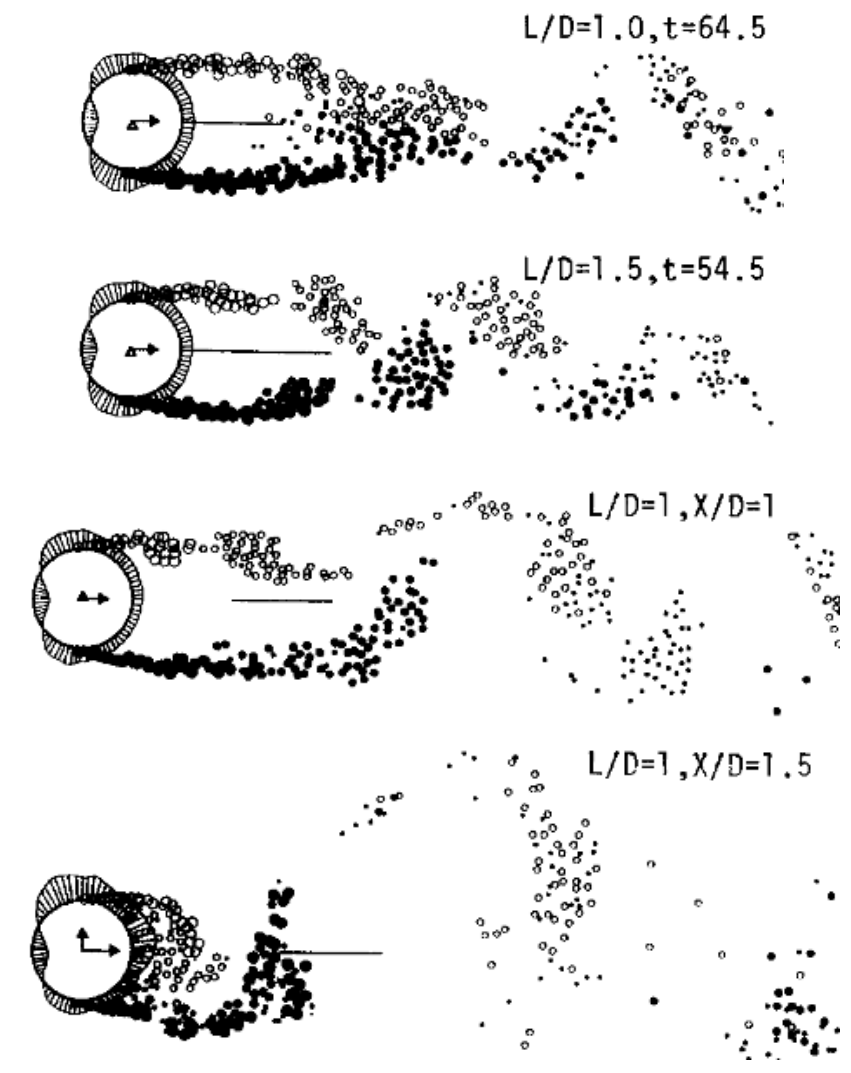

Figura 1.7: Visualização do escoamento de cilindro com splitter plate solucionado a partir do método dos vórtices discretos. Reprodução de Kawai (1990)

do número de Strouhal St em relação ao número de Reynolds Re e os resultados foram muito interessantes, pois esperava-se um comportamento semelhante ao encontrado por Kawai (1990) e o que foi obtido foram resultados de St muitos próximos aos encontrados por Gerrard (1966) e Apelt, West e Szewczyk (1973), apesar do baixo valor do número de Reynolds. Com isso, pode-se concluir que o número de Strouhal pode ter uma independência do número de Reynolds em uma faixa maior que foi encontrada por Igarashi (1982).

Um outro tópico importante que foi estudado e compreendido pois se assemelha muito ao escoamento com splitter plate é o escoamento de dois cilindros alinhados em relação ao escoamento. Em Meneghini (2001), um estudo numérico bidimensional foi realizado para estudar a intereferência da presença de um segundo corpo (neste caso um outro cilindro) na física do escoamento. Um importante fator que o trabalho destaca é a presença de um fenômeno chamado Inversão de Arrasto que ocorre quando para um certo GAP entre os corpos, o cilindro a jusante possui o seu vetor de arrasto no sentido contrário ao escoamento.

Quando o GAP ultrapassa um valor crítico, inicia-se novamente o despren- 
dimento de vórtices no cilindro a montante e afastando-se ainda mais os corpos rombudos, o escoamento ao redor deles torna-se igual ao de cilindros isolados. No trabalho de livre docência do autor há uma série de resultados do estudo de escoamentos em dois cilindros posicionados lado a lado e também na posição "tandem" avaliando as características do escoamento com a variação da distância entre os corpos (MENEGHINI, 2002).

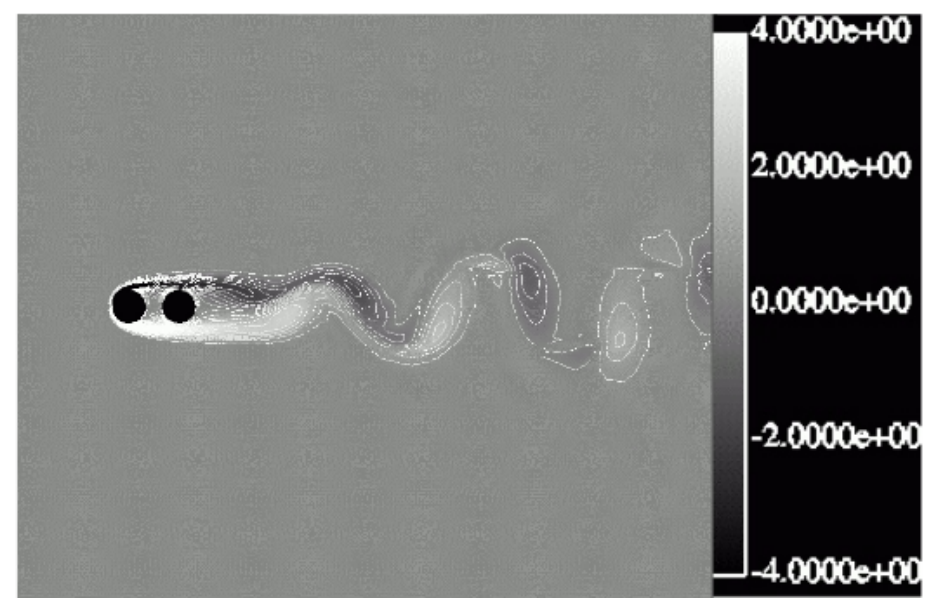

Figura 1.8: Contornos de Vorticidade no escoamento de dois cilindros alinhados em 'Tandem' (MENEGHINI, 2002)

Outra importante referência a ser mencionada é o trabalho desenvolvido por Carmo (2005) que realizou uma série de simulações numéricas com dois cilindros alinhados. Seu trabalho foi importante na apresentação do método dos elementos espectrais utilizad o neste trabalho. Na seção 2.3.1 encontra-se um breve resumo da elaboração deste método.

Atualmente, os estudos com splitter plates já são consolidados, iniciando agora uma nova etapa de desenvolvimento. Em todas as referências acima, o dispositivo foi colocado paralelo ao eixo do escoamento, no eixo de simetria do cilindro nesta direção. Como os oceanos possuem variações na direção do escoamento, o splitter plate não poderá ficar fixo em relação ao cilindro pois não causará o benefício apropriado. Assim, Assi, Bearman e Kitney (2009), Shukla, Govardhan e Arakeri (2009) desenvolveram métodos do splitter plate estar preso ao cilindro, mas livre para rotacionar em torno do eixo do corpo, alinhando-se ao escoamento. Outro segmento de desenvolvimento é estudo de variações da geometria do dispositivo a jusante do cilindro, como exemplos, podemos citar dois splitter plates paralelos, onde além do comprimento e do GAP seria necessário a distância relativa entre as duas placas e também splitter plates inclinados ao escoamento, onde seria inserido como variável de projeto, o ângulo relativo entre os mesmos.

Entretanto, com a inserção de novas variáveis de projeto, a quantidade de 


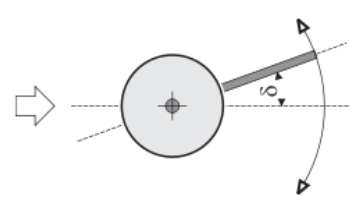

SINGLE SPLITTER PLATE

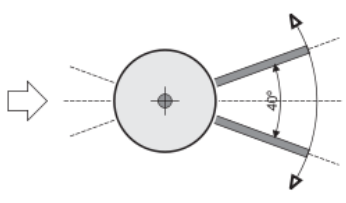

DOUBLE SPLITTER PLATES

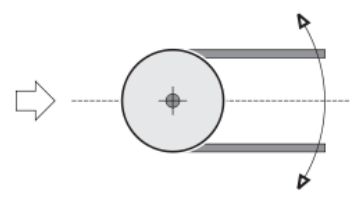

PARALLEL PLATES

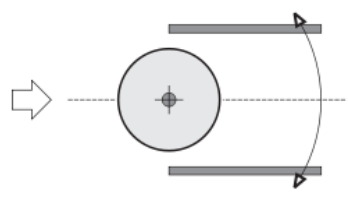

PARALLEL PLATES WITH GAP

Figura 1.9: Configurações de disposição de splitter plates (ASSI; BEARMAN; KITNEY, 2009)

simulações tornou-se excessivamente elevada, sendo praticamente inviável conseguir realizar simulações numéricas de todos os casos. Neste ponto, o método adjunto entrará futuramente como uma importante ferramenta pois sua formulação descrita nos capítulos 4 e 4.3 necessita apenas de uma solução base e uma solução adjunta, para determinar o gradiente de sensibilidade das variáveis, independendo do número de variáveis de projeto. 


\section{MeTODOLOGIA E FUNDAMENTOS TEÓRICOS}

\subsection{Introdução}

Neste capítulo estão descritas a metodologia e os fundamentos relevantes para a modelagem numérica e solução do escoamento ao redor do corpo rombudo. A abordagem não será apronfundada pois não é o escopo deste trabalho, porém, todas as referências adequadas para cada tópico serão mencionadas para eventuais pesquisas do leitor.

\subsection{Modelo Matemático do Escoamento}

No que se refere ao escoamento ao redor de cilindros com splitter plate, o objeto de estudo deste trabalho corresponde ao escoamento incompressível e viscoso, além de se considerar o fluido como sendo newtoniano, ou seja, a tensão cisalhante é proporcional ao gradiente da velocidade na direção normal de cada componente, sendo essa constante conhecida como viscosidade dinâmica. Assim, é possível modelar o problema em questão utilizando as equações de Navier Stokes.

As equações de Navier Stokes na forma diferencial descrevem o movimento dos fluídos Newtonianos-Stokerianos. Sua formulação é baseada em relações das taxas de variação (derivadas) ou fluxos das variáveis de interesse (No caso, velocidade e pressão):

$$
\left\{\begin{array}{r}
\frac{\partial u}{\partial t}+(u \cdot \nabla) u+\frac{\nabla p}{\rho}-\nu \nabla^{2} u=0 \\
\nabla \cdot u=0
\end{array}\right.
$$

onde $u=(u, v, w)$ é o vetor velocidade, $t$ é o tempo, $p$ é a pressão, $\rho$ é a densidade e $\nu=\frac{\mu}{\rho}$ é a viscosidade cinemática do fluido. É possível adimensionar 
as equações utilizando as seguintes relações:

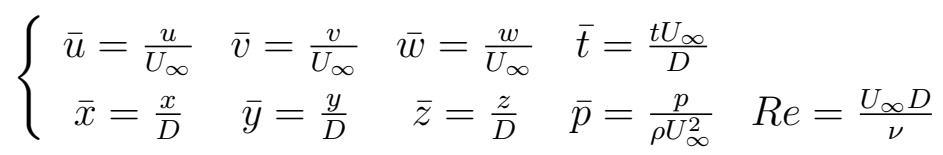

onde $U_{\infty}$ é a velocidade ao longe, D é a dimensão característica do corpo, que neste trabalho, é o diâmetro do cilindro e Re é o número de Reynolds. O desenvolvimento a seguir utilizará as variáveis adimensionalizadas. As equações de Navier-Stokes podem então ser escritas na sua forma adimensional:

$$
\left\{\begin{array}{c}
\frac{\partial u}{\partial t}+(u \cdot \nabla) u=-\nabla p+\frac{1}{R e} \nabla^{2} u \\
\nabla u=0
\end{array}\right.
$$

A maioria dos problemas que utilizam as equações acima não possuem solução geral conhecida. De fato, as soluções analíticas são disponíveis apenas para uma coleção limitada de casos de interesse, sendo particularidade apenas de problemas mais simples.

Como não é o caso do presente trabalho, para solucionar as equações, adotase a resolução numérica, utilizando recursos computacionais e modelos baseados em volumes finitos.

\subsection{Solução Numérica do Escoamento}

Para a solução numérica do escoamento, foi um escolhido um software conhecido como NEKTAR (KARNIADAKIS G.E.; SHERWIN, 1999), que possui um código fonte aberto fornecido pelo Dr. Spencer J. Sherwin (Imperial College, LondresInglaterra). $\mathrm{O}$ algoritmo presente no programa possui a vantagem de ter uma convergência exponencial com alta resolução espacial, provenimente dos métodos espectrais puros e também a possibilidade de refinamento local e flexibilidade geométrica, proveniente dos elementos finitos (CARMO, 2005).

Uma vantagem deste software é justamente o acesso aos códigos fonte, proporcionando alterações que possam ser necessárias para o trabalho. A seguir, será descrito o modelo numérico contido neste código. 


\subsubsection{Método dos elementos espectrais}

Nesta seção há um breve resumo sobre a modelagem matemática do método dos elementos espectrais. O foco deste trabalho está na modelagem do método adjunto e uso do NEKTAR para solução do problema proposto e portanto, para um maior aprofundamento dos métodos espectrais, recomenda-se a leitura de Carmo (2005) e Karniadakis G.E.; Sherwin (1999).

O método de elementos espectrais é uma união de métodos espectrais puros com o método de elementos finitos, empregando na formulação de elementos finitos, funções de base espectrais. Das funções espectrais, vem a convergência exponencial e a alta resolução, devido à alta ordem das funções de aproximação. Dos elementos finitos vem a divisão do domínio em elementos, que permite refinamento local e flexibilidade geométrica (CARMO, 2005).

Os métodos de elementos finitos (FEM) foram os primeiros métodos numéricos que permitiram a solução de problemas em geometrias complexas com certa facilidade. Depois de anos de evolução e estudo, este método hoje é utilizado na solução de praticamente qualquer tipo de equação diferencial parcial e sistemas de equações diferenciais parciais.

Os métodos espectrais (do inglês: Spectral Methods - SM) derivam de métodos analíticos de solução de equações diferenciais parciais que apresentam soluções baseadas em expansões em série de funções ortogonais. Estas funções são suaves e o erro da equação diferencial é minimizado segundo critérios pré-estabelecidos. A vantagem deste método é a convergência exponencial que possibilita a solução do problema com relativamente poucos graus de liberdade. Entretanto, geometrias complexas são difíceis de serem tratadas com esta abordagem (CARMO, 2005).

A partir disso, podem-se observar nos métodos numéricos utilizados para a simulação de escoamentos duas tendências predominantes. Por um lado, existem métodos de baixa ordem para simulação de problemas em geometrias complexas e problemas de engenharia envolvendo modelos físicos avançados (modelos de turbulência do tipo k- $\epsilon$ por exemplo). Na outra frente, pesquisas envolvendo simulação numérica direta (DNS) só são possíveis com métodos de ordem superior. Os métodos de elementos espectrais (SEM) procuram justamente conciliar estas duas tendências (CARMO, 2005).

Uma outra questão relevante é a simulação durante longos intervalos de tempo. Nesta situação, uma resolução espacial alta é essencial para minimizar os erros (KARNIADAKIS G.E.; SHERWIN, 1999), o que é o caso deste trabalho onde se 
estudam séries temporais de grandezas num escoamento ao redor de cilindros.

A seguir, será feita uma breve exposição dos fundamentos teóricos que norteiam o método utilizado.

\subsubsection{Método dos Resíduos Ponderados e formulação de Garlekin}

Ao se aproximar numericamente a solução exata de uma equação, tipicamente o que se faz é substituir uma expansão infinita, que é a solução exata, por uma representação dada por um conjunto finito de funções conhecidas. Tal aproximação, portanto, é incapaz de satisfazer a equação diferencial em todos os pontos do domínio de interesse. Ao invés disso, impõe-se que esta aproximação tenha que satisfazer um número finito de condições. A escolha destas condições é que determina o tipo de método numérico. O método de resíduos ponderados consiste em utilizar funções de peso (ou ponderação) na forma integral ou forma fraca da equação diferencial em questão para se chegar a aproximações válidas da solução (CARMO, 2005).

Para descrever este método, considera-se uma equação diferencial linear num domínio $\Omega$ denotada por:

$$
L(u)=0
$$

sujeita a condições de contorno e condições iniciais adequadas. Assume-se que a solução $u(x, t)$ pode ser representada com uma dada precisão por uma solução aproximada da forma (KARNIADAKIS G.E.; SHERWIN, 1999):

$$
u^{\delta}(x, t)=u_{0}(x, t)+\sum_{i=1}^{N_{g l}} \hat{u}_{i}(t) \phi_{i}(x)
$$

Onde $\Phi_{i}(x)$ são funções analíticas chamadas funções de base ou funções de forma, $\hat{\mathrm{u}}_{i}(t)$ são os $N_{g l}$ coeficientes desconhecidos e $u_{0}(\mathrm{x}, \mathrm{t})$ é selecionada de modo a satisfazer as condições de contorno e condições iniciais. A substituição da aproximação 2.5 na equação 2.4 produz um resíduo não-nulo, $\mathrm{R}$, tal que:

$$
L\left(u^{\delta}\right)=R\left(u^{\delta}\right)
$$


A fim de estabelecer uma maneira única de determinar os coeficientes $\hat{u}_{i}(t)$, é imposta uma restrição ao resíduo $R$ de modo que a equação 2.6 reduza-se a um sistema de equações diferenciais ordinárias em $\hat{u}_{i}(t)$. Se a equação diferencial original 2.4 é independente do tempo, então os coeficientes $\hat{\mathrm{u}}_{i}$ podem ser determinados diretamente da solução de um sistema de equações algébricos (CARMO, $2005)$.

Definindo o produto interno $(f, g)$ no domínio $\Omega$ :

$$
(f, g)=\int_{\Omega} f(x) g(x) d x
$$

a restrição imposta a $R$ é que o produto interno do resíduo com uma função peso é igual a zero, ou seja, ortogonalidade:

$$
\left(v_{j}(x), R\right)=0, j=1, \ldots, N_{g l}
$$

A função $v_{j}(x)$ é a função peso e é daí que vem o nome da técnica.

Se o método apresenta convergência, $R(x)$ tenderá a zero desde que a solução $u^{\delta}(x, t)$ tenda para a solução exata $u(x, t)$ na medida em que $N_{g l} \longrightarrow$ inf . A natureza do esquema é determinado pela escolha das funções de base $\Phi i(x)$ e das funções peso $v_{j}$.

Dentre as várias escolhas possíveis, o método utilizado neste trabalho é o método de Galerkin. Nele as funções de peso são iguais às funções de base, ou seja, $v_{j}=\Phi_{j}$. Outros métodos além do método de Galerkin são brevemente descritos em (KARNIADAKIS G.E.; SHERWIN, 1999).

Na formulação do tipo Galerkin, condições de contorno do tipo Dirichlet têm que ser especificadas explicitamente enquanto condições de contorno do tipo Neumann são tratadas implicitamente, como parte da formulação, através do uso de integração por partes e de uma função teste que se anule nas partes da fronteira onde condições de contorno do tipo Dirichlet são especificadas (KARNIADAKIS G.E.; SHERWIN, 1999). Por isso, as condições de contorno do tipo Neumann são também chamadas de naturais enquanto as do tipo Dirichlet são também chamadas de essenciais.

Para tratar condições de contorno essenciais não-homogêneas, tendo em vista que as funções de teste são nulas nas regiões onde tais condições são definidas, 
é preciso que a base utilizada para aproximar a solução contenha outras funções que sejam não nulas nestes contornos. Sem isso, seria impossível satisfazer estas condições de contorno do problema. Assim, a solução aproximada $u^{\delta}$ é composta de uma parcela conhecida $u^{D}$, que satisfaz as condições de contorno essenciais, e uma parcela homogênea desconhecida, $u^{H}$, que se anula nos contornos com condição do tipo Dirichlet, ou seja:

$$
u^{\delta}=u^{D}+u^{H}
$$

Desse modo, o mesmo conjunto de funções agora é usado para representar a solução homogênea $u^{H}$ e a função de teste $v$.

Algumas propriedades da formulação de Galerkin que tornam o seu uso interessante são unicidade de solução, ortogonalidade do erro (Eq. 2.8) em relação ao espaço de funções de peso, solução que minimiza a norma de energia do erro e equivalência de bases polinomiais no que diz respeito à norma de energia. Esta última característica tem a implicação importante de que a estimativa de erro é independente do tipo de expansão polinomial, dependendo somente do espaço polinomial (KARNIADAKIS G.E.; SHERWIN, 1999).

\subsubsection{Resolução das Equações de Navier Stokes aplicando o método dos elementos espectrais}

A solução numérica das equações de Navier-Stokes apresenta diversas dificuldades, destacando principalmente a existência do termo não linear. No método a seguir, o termo não linear é tratado de maneira explícita e com um intervalo de tempo $(\Delta t)$ adequado este problema é solucionado.

Outra dificuldade encontrada a maneira como a pressão e a velocidade estão acopladas nas equações, o que não permite que as duas variáveis sejam aproximadas de maneira independente. Para solucionar este problema, a aproximação da solução pelos espaços discretos deve satisfazer uma condição conhecida como infsub ou div-stability descritas em Karniadakis G.E.; Sherwin (1999)

\subsubsection{Condições de contorno e avanço no tempo}

As condições de contorno apropriadas para o escoamento externo ao redor de um corpo rombudo serão definidas a seguir, seguindo os trabalhos de Karniadakis, Israeli e Orszag (1991). 
Iniciando com as condições de contorno para velocidade, é possível afirmar que ao longe, onde o corpo e a esteira de vórtices não interfere no campo de velocidades, o escoamento pode ser considerado como não perturbado e a velocidade é igual a $U_{\infty}$.

No cilindro, é imposta condição de não escorregamento e a velocidade é nula. Já para o splitter plate é colocada a condição de parede invíscida, ou seja, é imposto que o produto escalar com a normal ser nula.

$$
\{\mathbf{u} \cdot \mathbf{n}=0
$$

A justificativa para seu uso ao invés da condição de parede é a redução significativa do custo computacional da simulação sem prejudicar os resultados no corpo rombudo. Segundo Igarashi (1982), splitter plates com comprimento na faixa $l / d \leq 1,76$ possuem forças de arrasto pequenas em relação ao cilindro. Como este trabalho irá analisar com splitter plates com comprimentos na faixa $l / d \leq 1,5$, essa condição pode ser usada, sendo uma prática já adotada na comunidade científica.

Na região de saída de fluido, tipicamente influenciada pela esteira, a derivada da velocidade na direção normal do contorno é nula, ou seja, $\frac{\partial u}{\partial n}=0$.

Descrevendo agora as condições de pressão, temos que na saída também se impõe uma condição do tipo Neumann como na velocidade, isto é, $\frac{\partial p}{\partial n}=0$. Quanto à condição de contorno da pressão na parede, a literatura apresenta diversas opiniões sobre o assunto.

Vale lembrar que não existe uma condição para pressão na parede. Portanto, a pressão na parede (seja invíscida ou não) é conseqüência do escoamento. Tradicionalmente, adota-se para a pressão um gradiente nulo:

$$
\left\{\left.\frac{\partial p}{\partial n}\right|_{\text {parede }}=0\right.
$$

Entretanto, segundo Karniadakis, Israeli e Orszag (1991), adotar essa condição de contorno significa prejudicar a tentativa de se obter uma precisão melhor que a primeira ordem do tempo, sobretudo quando a parede possui curvatura. Para solucionar isso, uma condição de contorno mais elaborada é adotada, baseando-se em uma condição de Neumann de alta ordem.

Esta condição de contorno da pressão diz que a derivada da equação de equilíbrio de momento linear na direção normal da fronteira será nula. No algoritmo 
utilizado, esta condição foi empregada também nas regiões de escoamento ao longe. Uma explicação mais detalhada dessa condição será dada a seguir junto com a descrição do avanço no tempo.

Ao se resolver as equações de Navier-Stokes no tempo, é necessário adotar uma discretização temporal. O mais usual em escoamentos incompressíveis é fazer a discretização no tempo independente da discretização no espaço. O método de elementos espectrais permite uma resolução muito alta no espaço mas isso de nada adianta se a resolução temporal não for compatível com esta precisão (CARMO, 2005).

O método proposto para a resolução das equações de Navier-Stokes transitórias é o time-splitting que permite o uso de preciões de ordem superior. Assim, reescrevendo a equação 2.3 , temos que:

$$
\left\{\frac{\partial u}{\partial t}-N(u)=-\nabla p+\frac{1}{R e} L(u)\right.
$$

Onde:

$$
\left\{\begin{aligned}
N(u)=-(u \nabla) u & =\frac{1}{2}[u \nabla u+\nabla(u u)] \\
L(u) & =\nabla^{2} u
\end{aligned}\right.
$$

Os termos $\mathbf{N}(\mathbf{u})$ e $\mathbf{L}(\mathbf{u})$ representam o termos convectivo e difusivo respectivamente. Integrando a equação $2.12 \mathrm{em}$ um passo de tempo $\Delta t$ temos que:

$$
u^{n+1}-u^{n}=-\int_{t_{n}}^{t_{n+1}} \nabla p d t+\frac{1}{R e} \int_{t_{n}}^{t_{n+1}} L(u) d t+\int_{t_{n}}^{t_{n+1}} N(u) d t
$$

Sendo $t_{n}=n \Delta t$. O termo não-linear $(N(u))$ será aproximado por um esquema explícito de ordem $J_{e}$ da família de Adams-Bashforth, pois possui melhor eficiência (CARMO, 2005):

$$
\int_{t_{n}}^{t_{n+1}} N(u) d t=\Delta t \sum_{q=0}^{J_{e}-1} \beta_{q} N\left(u^{n-q}\right)
$$

Onde $\beta_{q}$ são os pesos dados pela tabela 2.1, que são função da ordem de integração escolhida.

Já os termos lineares $\mathbf{L}(\mathbf{u})$ são aproximados de forma implícita por razões 
Tabela 2.1: Coeficientes dos algoritmos das família Adams-Bashforth

\begin{tabular}{llll}
\hline Coeficiente & $\mathbf{1}^{\boldsymbol{a}}$ ordem & $\mathbf{2}^{\boldsymbol{a}}$ ordem & $\mathbf{3}^{\boldsymbol{a}}$ ordem \\
\hline$\beta_{0}$ & 1 & $3 / 2$ & $23 / 12$ \\
$\beta_{1}$ & 0 & $-1 / 2$ & $-16 / 12$ \\
$\beta_{2}$ & 0 & 0 & $5 / 12$
\end{tabular}

de estabilidade. Será utilizado um esquema de ordem $J_{i}$ da família de AdamsMoulton (CARMO, 2005), chegando a:

$$
\int_{t_{n}}^{t_{n+1}} L(u) d t=\Delta t \sum_{q=0}^{J_{i}-1} \gamma_{q} L\left(u^{n+1-q}\right)
$$

Onde $\gamma_{q}$ são os pesos dados pela tabela 2.2, que são função da ordem de integração escolhida.

Tabela 2.2: Coeficientes dos algoritmos das família Adams-Moulton

\begin{tabular}{llll}
\hline Coeficiente & $\mathbf{1}^{\boldsymbol{a}}$ ordem & $\mathbf{2}^{\boldsymbol{a}}$ ordem & $\mathbf{3}^{\boldsymbol{a}}$ ordem \\
\hline$\gamma_{0}$ & 1 & $1 / 2$ & $5 / 12$ \\
$\gamma_{1}$ & 0 & $1 / 2$ & $8 / 12$ \\
$\gamma_{2}$ & 0 & 0 & $-1 / 12$
\end{tabular}

Por fim, o termo da pressão é reescrito da seguinte maneira:

$$
\int_{t_{n}}^{t_{n+1}} \nabla p d t=\Delta t \nabla \bar{p}^{n+1}
$$

Onde $\bar{p}^{n+1}$ é um campo escalar que assegura que o campo de velocidades final é incompressível ao final do passo de tempo $(n+1)$.

Com esta notação, a discretização temporal será realizada em 3 etapas, na seguinte forma:

$$
\begin{gathered}
\frac{\hat{u}-u^{n}}{\Delta t}=\sum_{q=0}^{J_{e}-1} \beta_{q} N\left(u^{n-q}\right) \\
\frac{\hat{\hat{u}}-\hat{u}}{\Delta t}=-\nabla \bar{p}^{n+1}
\end{gathered}
$$




$$
\frac{u^{n+1}-\hat{\hat{u}}}{\Delta t}=\nu \sum_{q=0}^{J_{i}-1} \gamma_{q} L\left(u^{n+1-q}\right)
$$

com condições essenciais em $u_{0}$ :

$$
u^{n+1}=u_{0}
$$

Nas equações 2.18 a 2.20, os termos $\hat{u}$ e $\hat{\hat{u}}$ são campos de velocidade intermediários. A equação 2.18 é uma expressão explícita e todos os termos são conhecidos. A equação 2.19 define o valor de $\hat{\hat{u}}$ que será utilizado para se calcular $u^{n+1}$ na equação 2.20 .

Entretanto, cabe ressaltar uma dificuldade adicional na equação 2.19. Esta equação possui duas icógnitas, $\hat{\hat{u}}$ e $\bar{p}^{n+1}$ e portanto é necessário uma equação adicional. Assim, assume-se que o campo de velocidades temporário $\hat{\hat{u}}$ satisfaz a equação da continuidade, assim:

$$
\nabla \cdot \hat{\hat{u}}=0
$$

Substituindo a equação 2.22 na equação 2.19, temos a expressão conhecida como equação de Poisson da pressão:

$$
\nabla^{2} \bar{p}^{n+1}=\nabla\left(\frac{\hat{u}}{\Delta t}\right)
$$

A diferenciação da equação 2.19 torna necessário impor condição de contorno adicional. Assim, é necessário impor as condições de contorno desta equação para se resolver o campo de pressões. Tomando a equação 2.14 integrada no tempo e multiplicando pelo vetor normal $(n)$, temos que:

$$
\int_{t_{n}}^{t_{n+1}} \frac{\partial u}{\partial t} \mathbf{n} d t=-\int_{t_{n}}^{t_{n+1}} \nabla p \mathbf{n} d t+\frac{1}{R e} \int_{t_{n}}^{t_{n+1}} L(u) \mathbf{n} d t+\int_{t_{n}}^{t_{n+1}} N(u) \mathbf{n} d t
$$

O termo interno da integral do lado esquerdo da equação 2.24 será reescrito da seguinte forma:

$$
\frac{\partial u}{\partial t} n=\frac{\partial}{\partial t}(u \cdot n)-u \cdot \frac{\partial n}{\partial t}
$$

O segundo termo do lado direito será nulo, uma vez que o domínio é fixo 
e portanto o vetor normal é invariante no tempo. O primeiro termo representa o fluxo mássico líquido do domínio. Como o escoamento é incompressível, sem fontes e sorvedouros no interior no domínio, a integral no contorno deste termo é nula.

Assim, o termo do lado esquerdo da equação 2.24 é nulo e substituindo pelas equações 2.15,2.16 e 2.17, chega-se a equação de Poisson:

$$
\frac{\partial \bar{p}^{n+1}}{\partial n}=n \cdot\left[\sum_{q=0}^{J_{e}-1} \beta_{q} N\left(u^{n-q}\right)+\frac{1}{R e} \sum_{q=0}^{J_{i}-1} \gamma_{q} L\left(u^{n+1-q}\right)\right]
$$

É possível observar que do lado direito da equação 2.26 possui termos no tempo $n+1$ que são incógnitas. Para resolver este problema e garantir estabilidade na solução, o termo difusivo será reescrito da seguinte forma:

$$
L(u)=\nabla^{2} u=\nabla(\nabla \cdot u)-\nabla \times(\nabla \times u)
$$

O primeiro termo, $\nabla(\nabla \cdot u)$, será tratado de forma implícita enquanto o segundo termo, $-\nabla \times(\nabla \times u)$, será tratado de forma explícita. Reescrevendo a equação 2.26:

$\frac{\partial \bar{p}^{n+1}}{\partial n}=n \cdot\left[\sum_{q=0}^{J_{e}-1} \beta_{q} N\left(u^{n-q}\right)+\frac{1}{R e} \sum_{q=0}^{J_{i}-1} \gamma_{q} \nabla\left(\nabla \cdot u^{n+1-q}\right)+\frac{1}{R e} \sum_{q=0}^{J_{i}-1} \gamma_{q}\left(-\nabla \times\left(\nabla \times u^{n-q}\right)\right)\right]$

Nesta equação, o termo $\gamma_{0} \nabla\left(\nabla \cdot u^{n+1}\right)$ pode ser igualado a zero, pois o requisito de incompresibilidade no passo $n+1$ faz com que $\nabla \cdot u^{n+1}=0$. Assim, todas as velocidades no passo $n+1$ da expressão são eliminadas e única incógnita será $\frac{\partial \bar{p}^{n+1}}{\partial n}$.

Abaixo segue o resumo todos os passos para o avanço do tempo:

1. Calcula-se o campo de velocidades de intermediário $\hat{u}$ com a equação 2.18

2. Calcula-se o campo de pressões $\bar{p}^{n+1}$ com a equação 2.23 e 2.28

3. Com a pressão, calcula-se o campo intermediário $\hat{\hat{u}}$ com a equação 2.19

4. Por fim, o campo de velocidades $u^{n+1}$ é calculado com a equação 2.20 


\subsection{Condições de setup}

As condições de setup para o escoamento ao redor de cilindro são muito simples de serem inseridas e estão disponíveis facilmente em qualquer software de CFD, como o NEKTAR.

Serão utilizadas as 4 condições de contorno descritas na seção 2.3.2.1 para as regiões descritas na figura 2.1. Adicionalmente deverá ser imposta uma condição inicial para o problema que de acordo com a experiência do grupo de pesquisa com o NEKTAR, acelera a instabilidade do escoamento, gerando uma pertubação no campo e consequentemente a oscilação do cilindro. Assim, inicia-se o NEKTAR com as seguintes componentes da velocidade no domínio interno:

$$
\left\{\begin{array}{l}
u=1.0 \\
v=0.1 \\
w=0.0
\end{array}\right.
$$

Vale ressaltar que o valor 1.0 para as velocidades significa que a mesma equivale ao valor de $U_{\infty}$, uma vez que os parâmetros foram adimensionalizados.
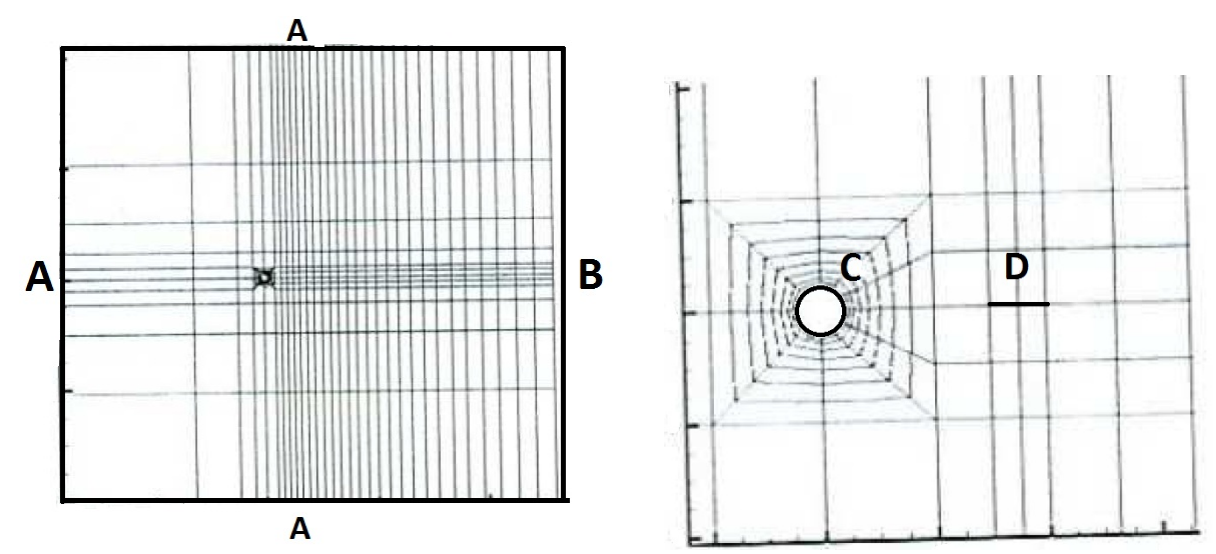

Figura 2.1: Regiões do domínio computacional onde serão inseridas as condições de contorno

\subsubsection{Condição de Entrada}

Na região da fronteira externa, denominada com a letra (A) na figura 2.1, a condição essencial imposta foi de velocidade adimensional unitária na componente 
x e nula nas componentes y e z.Assim, a condição imposta ficou:

$$
\left\{\begin{array}{l}
u=1.0 \\
v=0.0 \\
w=0.0
\end{array}\right.
$$

\subsubsection{Condição de Saída}

Na saída do domínio, representada pela letra (B) na figura 2.1, foi imposta uma condição natural, ou seja, a derivada das velocidades na fronteira na direção normal é nula. Neste caso, a direção normal é a própria direção x, pelo fato da malha ter o formato de um quadrilátero:

$$
\left\{\begin{array}{l}
\left.\frac{\partial V}{\partial x}\right|_{\text {fronteira }}=0 \\
p=0.0
\end{array}\right.
$$

\subsubsection{Condição de Parede}

Para a parede do cilindro (região C, figura 2.1) foi imposta a condição de parede, ou seja, velocidade nula na superfície:

$$
\left\{\begin{array}{l}
u=0.0 \\
v=0.0 \\
w=0.0
\end{array}\right.
$$

\subsubsection{Condição de Parede Invíscida}

A condição de parede invíscida é imposta apenas no splitter plate (região D, figura 2.1). As justificativas para esse uso estão na seção 2.3.2.1

\subsection{Malhas Computacionais}

Para a obtenção das malhas computacionais, utilizou-se o software GAMBIT, cuja licença está disponível nos computadores do NDF. Os elementos serão retangulares e a malha será estruturada. Pela quantidade de casos a serem estudados, um script de geração automática foi feito, onde a partir do posicionamento do splitter plate em relação ao cilindro, a malha era elaborada com dimensões padronizadas descritas neste script. 


\subsubsection{Testes de convergência e refino da malha}

Com o trabalho realizado por Serson e Meneghini (2010), foi possível otimizar a quantidade de elementos da malha computacional, bem como seu tamanho, além de calibrações do modelo proposto no software NEKTAR como o grau polinomial das funções de base. Os testes foram realizados com $R e=200$ pela facilidade de se encontrar resultados computacionais na literatura e utilizaram-se as mesmas condições de contorno descritas na seção 2.4.

Inicialmente criou-se uma malha preliminar de 416 elementos quadriláteros distribuídos em um domínio que se extendia na direção do escoamento (x), 37 diâmetros a montante e 52 diâmetros a jusante do cilindro. Já na direção transversal (y), o domínio foi de 84 diâmetros de extensão sendo o eixo do cilindro posicionado na metade, dividindo o domínio transversal em 42 diâmetros para cada lado.
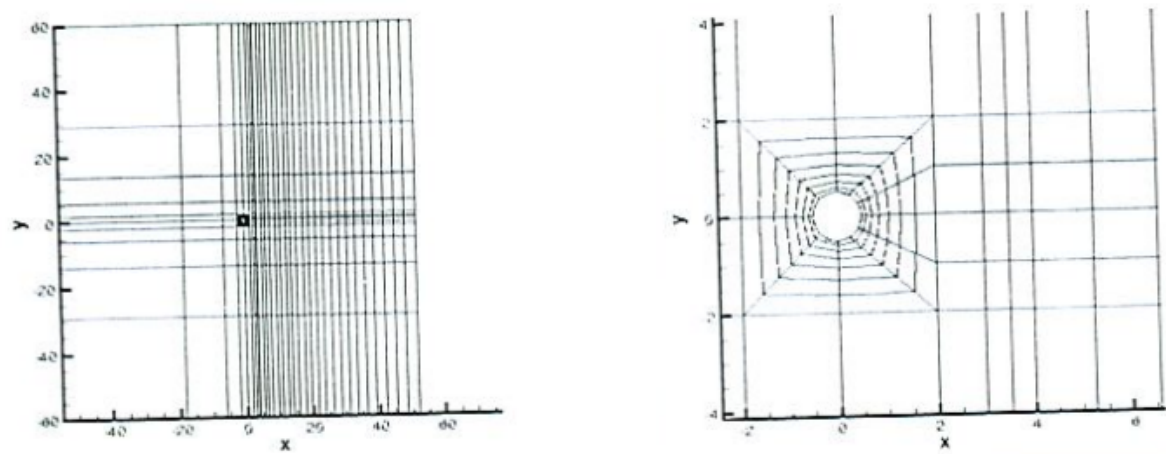

Figura 2.2: Malha computacional otimizada para as simulações numéricas (SERSON; MENEGHINI, 2010)

Foram realizados testes de convergência nessa malha preliminar, variando separadamente o grau polinomial das funções de base, a ordem de integração e passo de avanço no tempo, largura da malha e comprimento de entrada. Os testes com variação do comprimento de saída não foram realizados pois de acordo com Carmo (2005), a variação desta dimensão não produzia alterações significativas na convergência, sendo apenas importante para visualização da esteira de vórtices.

\subsubsection{Grau Polinomial das Funções de Base}

Em relação ao grau polinomial das funções de base, Serson e Meneghini (2010) realizaram simulações admitindo convergência quando a diferença para a simulação de grau imediatamente superior fosse menor que $0,1 \%$ para todos os parâmetros calculados que foram a média do coeficiente de arrasto, o valor do RMS do 
coeficiente de sustentação $C_{l}$, o número de Strouhal $S_{t}$ e o coeficiente de pressão na base do cilindro $C_{p b}$. A tabela abaixo apresenta os resultados das simulações e chegou-se ao grau 7 era o primeiro a atender os critérios de convergência (SERSON; MENEGHINI, 2010).

Tabela 2.3: Testes de convergência para grau polinomial

\begin{tabular}{lllll}
\hline Ordem & $\bar{C}_{D}$ & $C_{L}$ & $-\bar{C}_{p b}$ & $S t$ \\
\hline $\mathbf{6}$ & 1,3368 & 0,4811 & 0,9568 & 0,196 \\
$\mathbf{7}$ & 1,3337 & 0,4792 & 0,9550 & 0,196 \\
$\mathbf{8}$ & 1,3324 & 0,4788 & 0,9544 & 0,196 \\
$\mathbf{9}$ & 1,3323 & 0,4789 & 0,9545 & 0,196
\end{tabular}

\subsubsection{Discretização Temporal}

No método dos elementos espectrais, a discretização temporal é caracterizada por 2 parâmetros: Ordem de integração do tempo e o passo adimensional de avanço no tempo.

A ordem de integração temporal pode influenciar a precisão do método em duas áreas, sendo a primeira na forma como os termos convectivo e difusivo são tratados no avanço do tempo e a segunda, está relacionada na condição de contorno de pressão (KARNIADAKIS G.E.; SHERWIN, 1999).

O software NEKTAR utiliza a mesma ordem de integração temporal para o avanço no tempo e na condição de pressão. Todavia, cabe ressaltar que esses valores podem ser diferentes, sendo esta igualdade apenas uma condição do código de programação e não da teoria.

As ordens de integração testadas variaram de 1 a 3, pois são os valores permitidos pelo software. A tabela a seguir apresenta os resultados das simulações (SERSON; MENEGHINI, 2010).

Para o passo de avanço no tempo, utilizou-se o mesmo valor $\Delta t=0,005$ que fora utilizado nos testes do grau polinomial. Era esperado que este valor fosse suficiente para atender a convergência pois o próprio tratamento explícito do termo convectivo impõe que este valor seja pequeno para garantir a estabilidade do método numérico

Apenas para verificação da afirmação acima, foram realizados duas simula- 
Tabela 2.4: Testes de convergência para a ordem de integração temporal

\begin{tabular}{lllll}
\hline Ordem & $\overline{C_{D}}$ & $C_{L}$ & $-\bar{C}_{p b}$ & $S t$ \\
\hline $\mathbf{1}$ & 1,3410 & 0,4880 & 0,9635 & 0,197 \\
$\mathbf{2}$ & 1,3337 & 0,4791 & 0,9550 & 0,196 \\
$\mathbf{3}$ & 1,3336 & 0,4791 & 0,9549 & 0,196
\end{tabular}

ções, sendo a primeira com $\Delta t=0,005$ e a segunda com metade deste valor $\Delta t=0,0025$. A tabela a seguir apresenta os resultados (SERSON; MENEGHINI, 2010)

Tabela 2.5: Testes de convergência para para o avanço no tempo adimensional

\begin{tabular}{lllll}
\hline $\boldsymbol{\Delta} \boldsymbol{t}$ & $\bar{C}_{D}$ & $C_{L}$ & $-\bar{C}_{p b}$ & $S t$ \\
\hline $\mathbf{0 , 0 0 5 0}$ & 1,3337 & 0,4792 & 0,9550 & 0,196 \\
$\mathbf{0 , 0 0 2 5}$ & 1,3336 & 0,4791 & 0,9550 & 0,196
\end{tabular}

Observa-se na tabela 2.5 que diminuir o passo do tempo pela metade, não afetou significativamente o resultado dos parâmetros. Sendo assim, o valor de 0,005 será utilizado.

\subsubsection{Largura da Malha}

O próximos dois parâmetros a serem testados envolvem a geometria da malha, e não alguma propriedade direta do método numérico. Porém, é de grande importância verificar uma largura de malha apropriada para se evitar os efeitos de blocagem.

A blocagem é um fenômeno que ocorre em malhas estreitas onde a imposição da condição de velocidade ao longe será aplicada em uma região onde os efeitos da esteira de vórtices não podem ser desprezados.

No trabalho de Serson e Meneghini (2010) foi mantido o número de elementos pois na região em torno do cilindro, o gradiente não apresenta valores elevados, sem a necessidade portanto de um refinamento maior.

Sem essa necessidade de refinamento, para se manter o número de elementos, a região lateral foi apenas estreitada, o que não causou prejuízos as simulações. Esta estratégia também foi utilizada por Carmo (2005) e foi mantida neste trabalho 
A tabela 2.6 a seguir apresenta os resultados para simulações que variaram o comprimento da malha de 35 a 70 diâmetros.

Tabela 2.6: Testes de convergência para largura da malha

\begin{tabular}{lllll}
\hline Largura & $\overline{C_{D}}$ & $C_{L}$ & $-\bar{C}_{p b}$ & $S t$ \\
\hline $\mathbf{3 5}$ & 1,3354 & 0,4799 & 0,9531 & 0,196 \\
$\mathbf{4 2}$ & 1,3337 & 0,4792 & 0,9550 & 0,196 \\
$\mathbf{5 0}$ & 1,3325 & 0,4786 & 0,9565 & 0,196 \\
$\mathbf{6 0}$ & 1,3316 & 0,4782 & 0,9578 & 0,196 \\
$\mathbf{7 0}$ & 1,3311 & 0,4780 & 0,9586 & 0,196
\end{tabular}

\subsubsection{Comprimento de Entrada}

O comprimento de entrada (ou comprimento a montante do cilindro, como mencionado em Carmo (2005)) é a distância da região da condição de contorno de entrada em relação ao cilindro. Utilizando a mesma metodologia para o teste da largura da malha, a tabela abaixo apresenta os resultados para os comprimentos variando de 30 a 65 .

Tabela 2.7: Testes de convergência para largura da malha

\begin{tabular}{lllll}
\hline Largura & $\bar{C}_{D}$ & $C_{L}$ & $-\bar{C}_{p b}$ & $S t$ \\
\hline $\mathbf{3 5}$ & 1,3344 & 0,4796 & 0,9599 & 0,196 \\
$\mathbf{3 7}$ & 1,3337 & 0,4792 & 0,9550 & 0,196 \\
$\mathbf{4 5}$ & 1,3397 & 0,4772 & 0,9562 & 0,196 \\
$\mathbf{5 5}$ & 1,3382 & 0,4765 & 0,9550 & 0,196 \\
$\mathbf{6 5}$ & 1,3273 & 0,4761 & 0,9543 & 0,196
\end{tabular}

Para finalizar os testes de convergência, os resultados das simulações foram comparados a outros trabalhos realizados. Consultando as mesmas referências citadas em Meneghini (1993), a tabela a seguir apresenta a comparação de diversos resultados numéricos e experimentais.

Assim, a partir da geometria otimizada encontrada em Serson e Meneghini (2010), foi possível reproduzir o mesmo resultado para este trabalho, com 120 
diâmetros na direção transversal (o cilindro está posicionado no meio deste domínio) e 110 diâmetros na direção do escoamento (Sendo o cilindro posicionado a 55 diâmetros da entrada do domínio).

Tabela 2.8: Comparação dos Resultados entre diversas referências

\begin{tabular}{lcccc}
\hline & $\overline{C_{D}}$ & $C_{L}$ & $-\bar{C}_{p b}$ & $S t$ \\
\hline Teste de Convergência & 1,3282 & 0,4765 & 0,9550 & 0,196 \\
& & Numéricos & \\
Borthwick (1986) & 1,02 & & 0,188 \\
Braza M. Chassaing e Minh (1986) & 1,35 & & 0,20 \\
Carmo (2005) & 1,3331 & 0,478 & 0,954 & 0,196 \\
Lecointe, Piquet e Plantec (1987) & 1,30 & & 0,194 \\
Meneghini (1993) & 1,395 & 0,570 & 0,195 \\
& & Experimentais & \\
Roshko (1954a) & & & $0,17-0,19$ \\
Williamson (1991) & & & 0,196
\end{tabular}




\section{SimulaÇÕES NuMÉRICAS}

\subsection{Introdução}

Com a metodologia de projeto apresentada e todas as ferramentas devidamente calibradas, iniciaram-se as simulações numéricas com splitter plate para obtenção dos pontos de mínimo. Todas as simulações foram feitas no software NEKTAR utilizando a metodologia de construção da malha computacional desenvolvida por Serson e Meneghini (2010).

A busca foi feita para valores de $R e$ de 80 a 140, em intervalos de 20, com as condições de contorno descritas na seção 2.4. Este intervalo do adimensional foi escolhido de forma a evitar os efeitos de tridimensionalidade que ocorrem em faixas de $R e$ maiores, presentes na literatura.

Vale ressaltar também que no splitter plate foi imposta a condição de parede invíscida $(\mathbf{u} \cdot \mathbf{n}=0)$ que diminui significativamente o custo computacional sem prejudicar o cálculo das grandezas no cilindro.

Para comparação dos resultados, foi realizada uma simulação de referência, com o cilindro sem splitter plate para valor de Re investigado. Esta simulação serviu para avaliar quantitativamente a variação porcentual de cada grandeza avaliada.

\subsection{Simulação de Cilindro Isolado}

A simulação com cilindro isolado permitiu uma visualização do escoamento com destaque no desprendimento periódico de vórtices em cada lado do corpo rombudo. Para visualização dos resultados, utilizou-se um pós-processador disponibilizado para a pesquisa (TECPLOT). As figuras a seguir apresentam quadros temporais do escoamento, com a visualização da intensidade da vorticidade.

Na figura 3.1 observa-se claramente ciclos completos de desprendimento de vórtices. Com esse resultado, fica claro a proximidade do modelo que Gerrard 

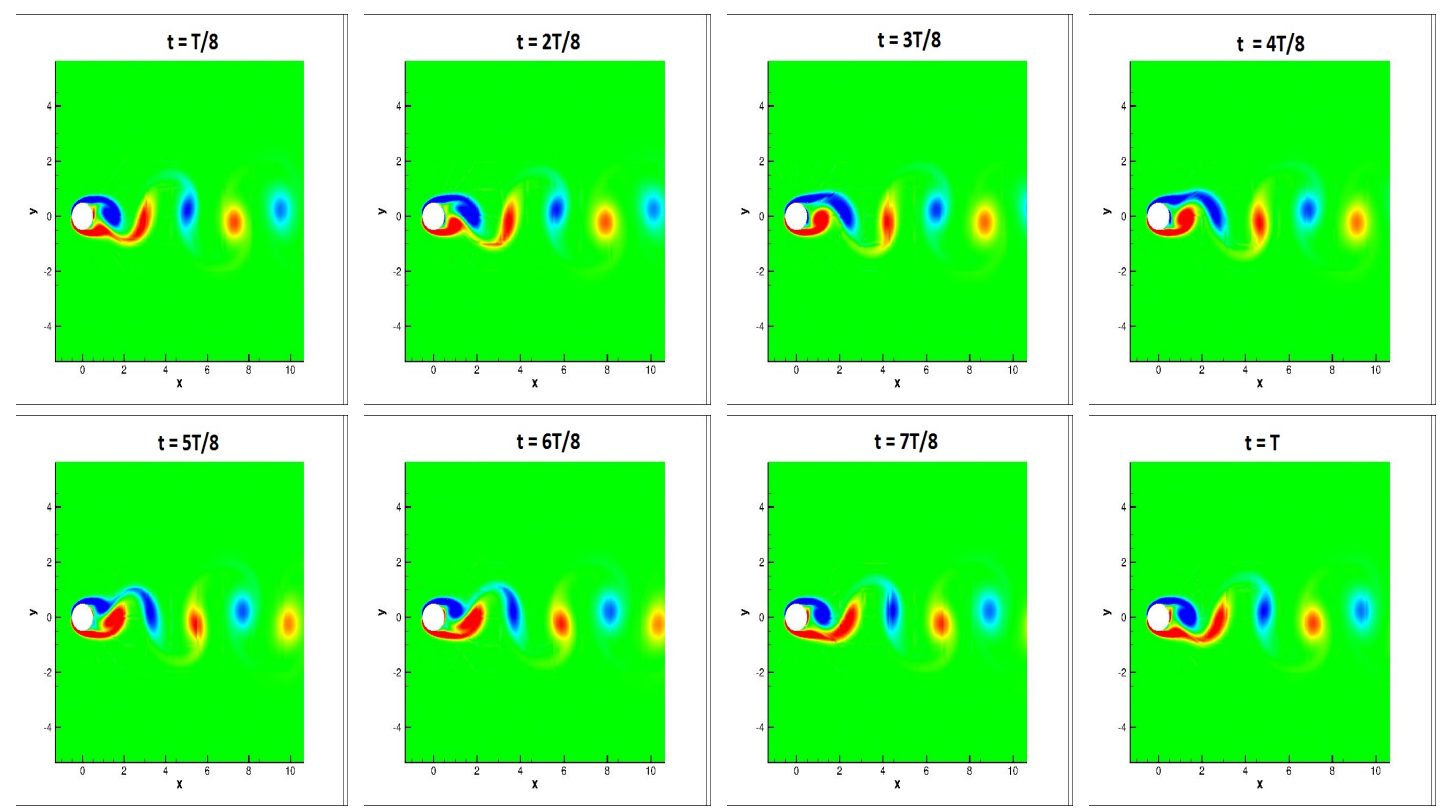

Figura 3.1: Quadros Temporais ao longo de um período T do Escoamento ao redor de um cilindro isolado $(R e=140)$

(1966) visualizou na década de 60. Apesar de não ter uma aplicação prática, o modelo auxilia a visualizar o fenômeno. Entretanto, não é possível distinguir e verificar com clareza onde exatamente cada componente do vórtice está localizada nesta figura.

Observando os dois primeiros quadros, pode-se perceber que houve uma separação do vórtice indicado pela cor vermelha. Neste momento, o próximo vórtice em vermelho ganhando intensidade entre vórtice azul e o cilindro. No terceiro e quarto quadros nota-se o a separação do vórtice vermelho e o início do desprendimento do vórtice em azul. No quinto e sexto quadros, é possível perceber o desprendimento do vórtice azul e a formação do próximo vórtice em vermelho, que inicia seu desprendimento nos sétimo e oitavo quadros, completando um período de oscilação. Após a simulação, foram construidos os gráficos em função do tempo dos coeficientes de arrasto e sustentação.

O comportamento do coeficiente de arrasto mostrou-se de acordo com o que foi visto em Igarashi (1982). Após um período de transição, o coeficiente passou a ter um movimento oscilatório, com um certo valor de frequência, que está diretamente ligada ao número de Strouhal do escoamento. Observa-se também que o coeficiente oscila em torno de um valor médio. Este valor $\overline{C_{d}}$ será a medida de mérito aplicada a este trabalho.

A sustentação possui o mesmo comportamento que o coeficiente de arrasto, com a diferença que o coeficiente oscila com o valor médio nulo, dada a simetria 


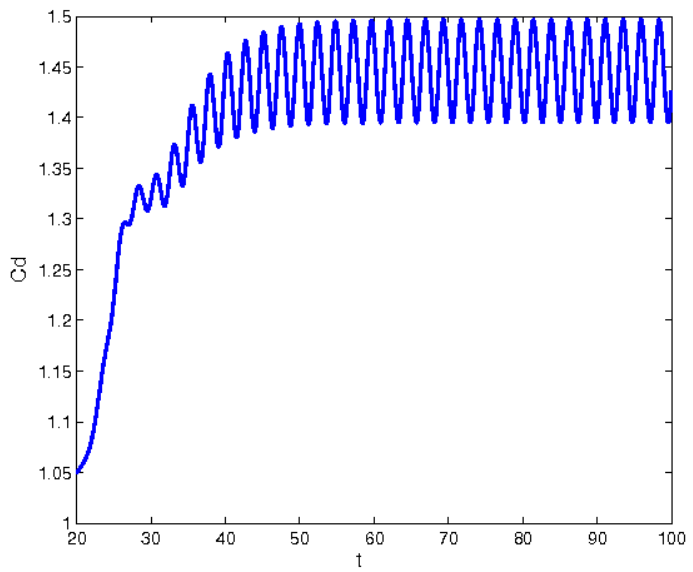

Figura 3.2: Visão Geral da Evolução do coeficiente de arrasto ao longo do tempo $(R e=140)$.

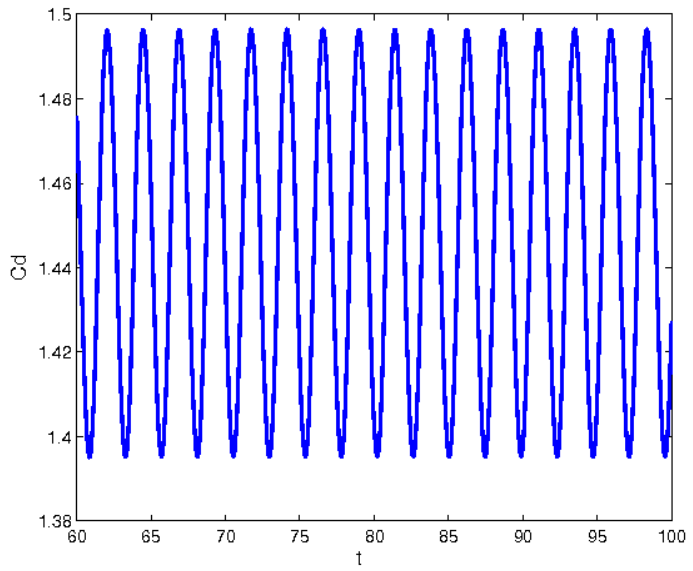

Figura 3.3: Detalhe na região do escoamento já desenvolvida $(R e=140)$

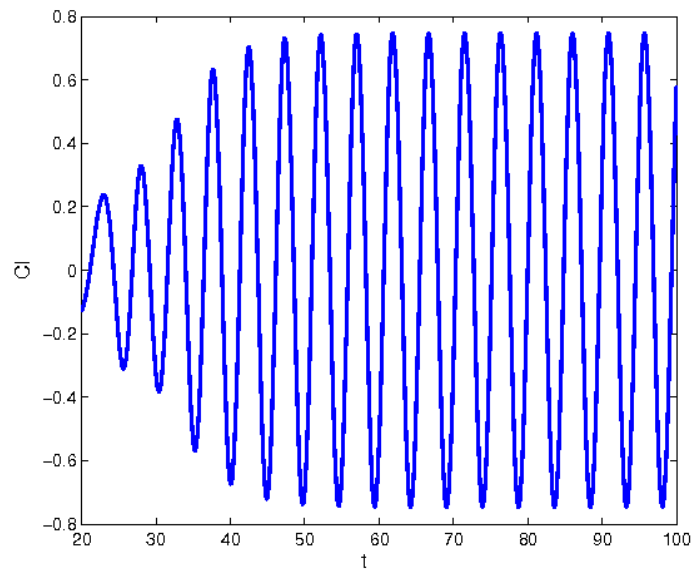

Figura 3.4: Visão Geral da Evolução do coeficiente de sustentação ao longo do tempo $(R e=140)$. 
do escoamento na média temporal, como observado nas figuras 3.4 e 3.5.

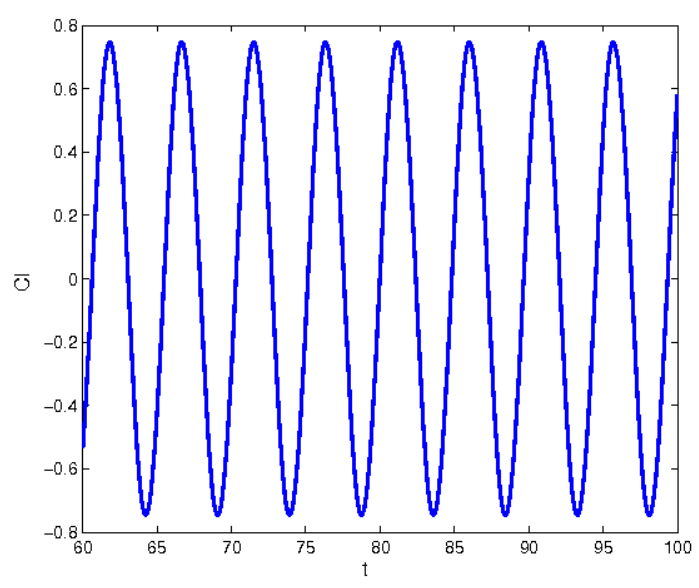

Figura 3.5: Detalhe na região do escoamento já desenvolvida $(R e=140)$.

\subsection{Simulações de Cilindros com Splitter Plate}

Nesta seção serão apresentados os resultados das diversas simulações realizadas considerando a presença do splitter plate. As tabelas 3.1 e 3.2 apresentam todos os testes realizados.

As simulações com splitter plate foram divididas em duas partes, sendo a primeira com a investigação em placas com GAP nulo em relação ao cilindro e depois splitter plates com GAP diferente de zero. Desta forma, o script de geração automática de malhas computacionais ficou mais simples, pois foi dividido em duas configurações diferentes de splitter plate.

Tabela 3.1: Simulações Realizadas com Splitter Plate - GAP Nulo

\begin{tabular}{|c|c|c|}
\hline$R e$ & $l / d$ & $g / d$ \\
\hline 140 & 0.29 & 0 \\
& 0.44 & \\
& 0.59 & \\
& 0.74 & \\
& 0.88 & \\
& 1.03 & \\
& 1.18 & \\
& 1.32 & \\
& 1.47 & \\
& 1.76 & \\
\hline
\end{tabular}


Tabela 3.2: Simulações Realizadas com Splitter Plate - GAP Variável

\begin{tabular}{|c|c|c|}
\hline$R e$ & $l / d$ & $g / d$ \\
\hline 80,100 & 0.5 & $0.6,0.8,1.0,1.2$ \\
120 e 140 & 1.0 & $1.4,1.6,1.8,2.0$ \\
& & $2.2,2.4,2.62 .8$ \\
& & $3.0,3.2,3.4,3.6,3.8$ e 4.0 \\
\hline
\end{tabular}

\subsubsection{Simulações com splitter plate posicionado na base do cilindro}

Para esta configuração de posicionamento do splitter plate, adotaram-se as mesmas dimensões de placa dos testes feitos em Igarashi (1982) e apresentados na figura 1.4 .

Como não está sendo considerado o arrasto do splitter plate, os resultados esperados serão diferentes do que os apresentados em Igarashi (1982). O autor explica que em configurações sem GAP, o arrasto tende a diminuir até um certo valor e depois voltará a aumentar pois o arrasto da placa irá tornar-se significante frente do cilindro.

Entretanto nas simulações realizadas, espera-se uma diminuição do arrasto até certo ponto e logo após isso, deveremos observar que os coeficientes fiquem com valores aproximadamente constantes por dois motivos: o primeiro devido as camadas cisalhantes já estarem interagindo longe o suficiente para não influenciar o escoamento perto do cilindro, portanto não influenciando significativamente os coeficientes calculados e segundo, devido a não contabilização do arrasto na placa, ou seja, não teremos parcelas que somarão ou diminuirão no arrasto total do sistema.

As figuras 3.6 e 3.7 apresentam os resultados obtidos nas simulações, onde podemos observar a variação dos coeficientes de arrasto e sustentação para diferentes comprimentos de splitter plate que estão apresentados na tabela 3.1.

É possível verificar claramente que o comportamento do escoamento foi de acordo com o esperado. A partir de $l / d=1,18$, o coeficiente médio do arrasto permaneceu praticamente constante, o que comprova a hipótese de Igarashi de que splitter plates longos tendem a perder seu efeito positivo pois a formação de vórtices está distante demais para causar mudanças significativas no escoamento (IGARASHI, 1982). Em relação a amplitude da sustentação, após o ponto de mínimo em $l / d=0,88$, o coeficiente de sustentação volta a oscilar. 


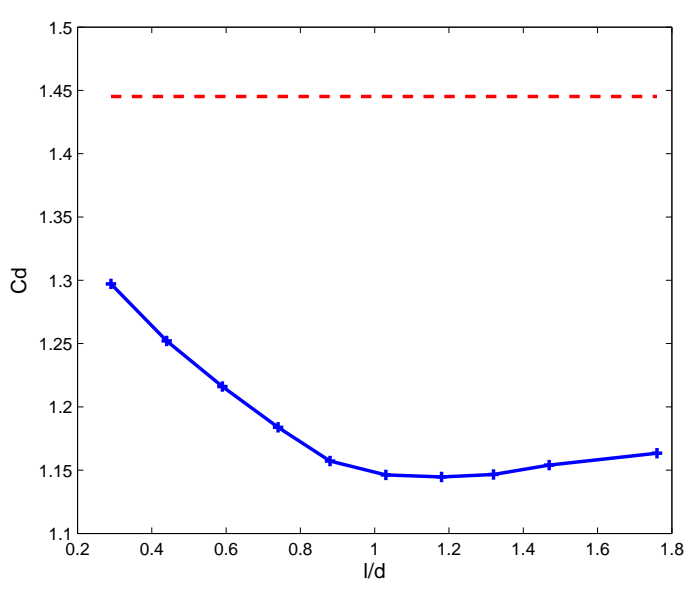

Figura 3.6: Valor do coeficiente de arrasto médio $\overline{C_{d}}$ para configuração de gap nulo em função de $l / d$, para $R e=140$. Linha vermelha, escoamento com cilindro isolado para comparação. Linha azul, escoamentos com splitter plate.

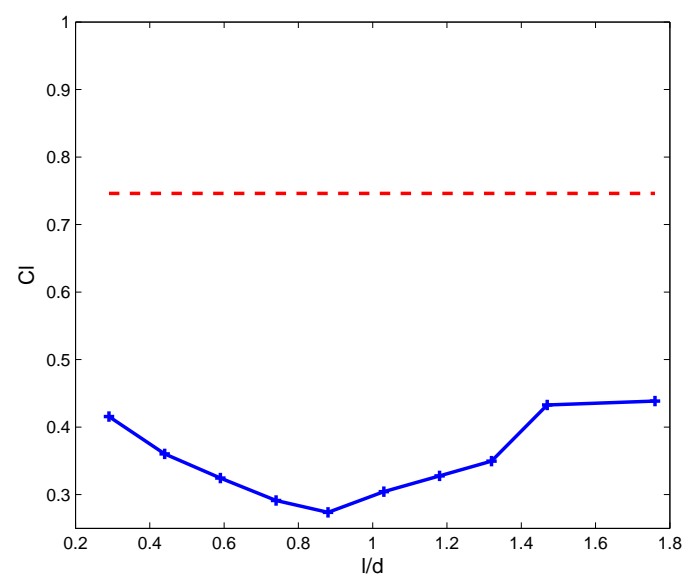

Figura 3.7: Valor da amplitude de $C_{l}$ para configuração de gap nulo em função de $l / d$, para $R e=140$. Linha vermelha, escoamento com cilindro isolado para comparação. Linha azul, escoamentos com splitter plate.

Avaliando quantitativamente, a simples presença da placa, independente do comprimento, causa reduções nos coeficientes de arrasto e sustentação, mas como o foco é a busca pela configuração otimizada, será focado apenas este ponto nas análises.

Na configuração ótima, o coeficiente médio de arrasto reduziu 19, 5\% no ponto $l / d=1,18$ e a amplitude de oscilação do coeficiente de sustentação diminuiu aproximadamente $60 \%$ para $l / d=0,88$. O resultado expressivo das reduções dos coeficientes mostra o potencial do splitter plate em diminuir o esforço realizado sobre o corpo.

A figura 3.8 apresenta ilustrações do escoamento para $l / d=1,0$ e GAP nulo. 
Observa-se que conforme o aumento do comprimento do splitter plate, a interação das camadas cisalhantes inicia-se mais afastada do cilidro, após o bordo de fuga da placa, ou seja, o comprimento de formação $l_{f}$ tornou-se maior.
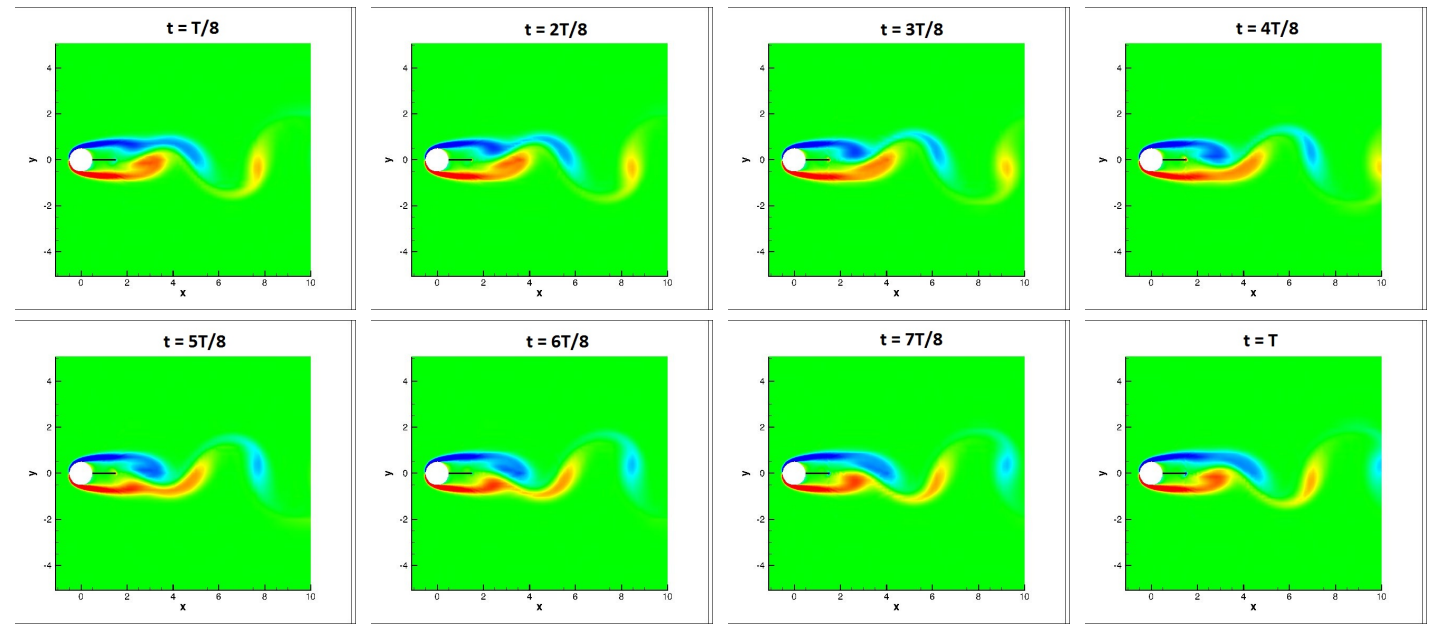

Figura 3.8: Quadros temporais da movimentação da vorticidade, $l / d=1,0 \mathrm{e}$ GAP nulo $(R e=140)$.

Outra forma de visualização é o gráfico com a variação no tempo dos coeficientes de arrasto e sustentação. As figuras 3.9 e 3.10 apresentam a redução do coeficiente de arrasto médio e o aumento do seu período de oscilação, ficando quase imperceptível a visualização dos picos e vales na escala da figura.

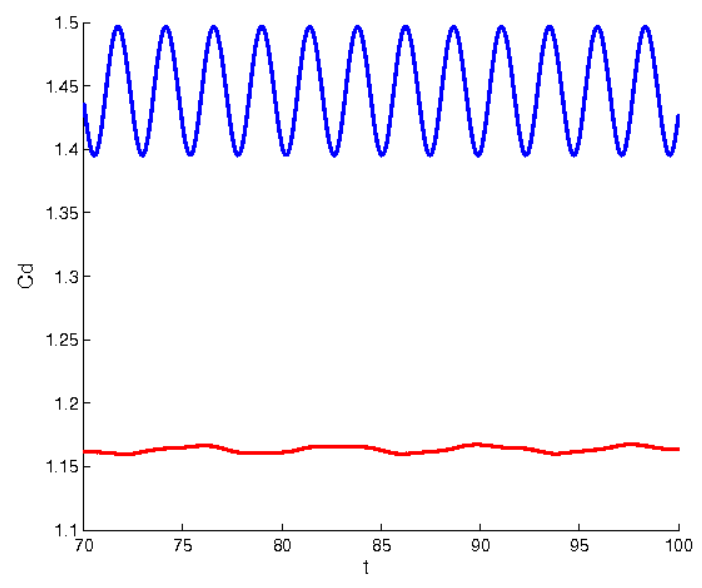

Figura 3.9: Valor do Coeficiente médio de arrasto $\overline{C_{d}}$ ao longo do tempo, para $R e=140, l / d=1.0$ e $g / d=0$. Linha azul, valores para o escoamento do cilindro sem splitter plate. Linha vermelha, com splitter plate na posição de $g / d=0$.

No caso da sustentação (figura 3.10), observa-se uma redução da máxima amplitude, mas o período de oscilação entre os escoamentos com e sem splitter plate tiveram pequenas variações. 


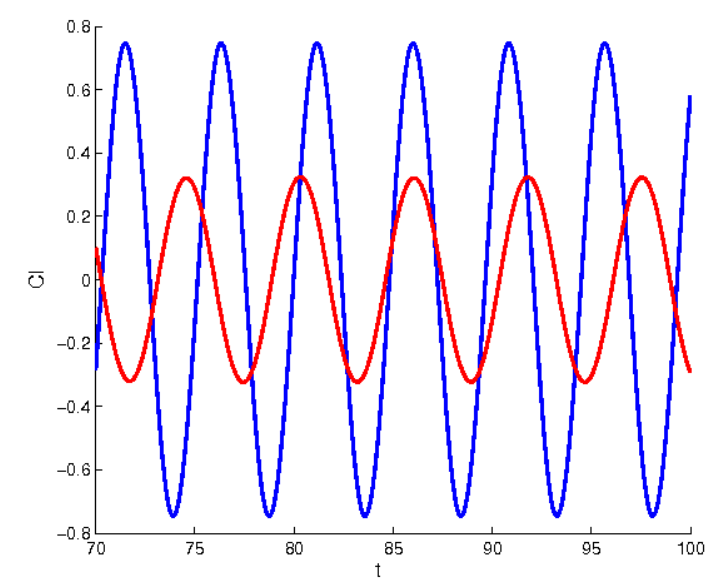

Figura 3.10: Máxima amplitude de $C_{l}$ ao longo do tempo, para $R e=140$, $l / d=1.0$ e $g / d=0$. Linha azul, valores para o escoamento do cilindro sem splitter plate. Linha vermelha, com splitter plate na posição de $g / d=0$.

\subsubsection{Simulações com splitter plate com variação do GAP}

As simulações com splitter plate variando o GAP foram feitas de maneira sistemática adotando o seguinte padrão: Foram definidos 2 comprimentos de placa a serem analisados, que foram $l / d=0,5$ e 1,0 . Mantendo o comprimento da placa constantes, geraram-se malhas computacionais com os valores de GAP apresentados na tabela 3.2 .

Após as simulações, os gráficos de coeficiente médio de arrasto e amplitude da sustentação eram feitos, para se visualizar em que intervalo estava ocorrendo a descontinuidade da curva, ou seja, quando as esteiras de vórtices voltavam a interagir nas proximidades do cilindro. Encontrado o intervalo, criavam-se malhas com valores de GAP dentro destas faixas, para se buscar um refino dos resultados e uma proximidade maior do ponto exato de mínimo.

\subsubsection{1 $\quad R e=80$}

As figuras 3.11 e 3.12 apresentam os resultados das variações de coeficientes de arrasto e sustentação para o comprimentos $l / d=0,5$.

Para comprimento de placa $l / d=0,5$, o ponto de menor arrasto médio ocorreu para um GAP $(g / d)$ de 2,0 com redução de 6,3\% em relação ao valor do coeficiente de arrasto médio para um escoamento com cilindro sem splitter plate. Após esse valor de mínimo, o arrasto médio inicia um aumento gradativo, convergindo para o valor do cilindro isolado.

No caso da sustentação, houve uma redução de 80,0\% da amplitude do coe- 


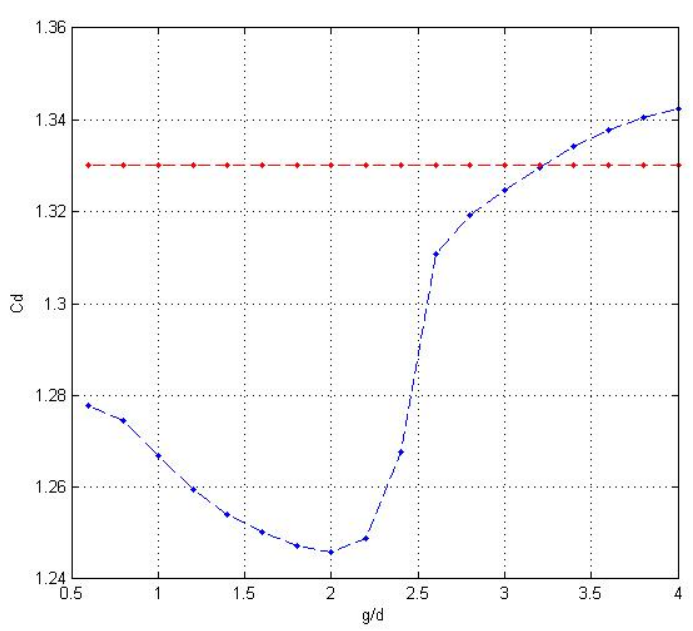

Figura 3.11: Coeficiente Médio de Arrasto $\overline{C_{d}}$ versus $g / d$, para $R e=80$ e $l / d=0,5$. Linha vermelha, valores para o escoamento do cilindro sem splitter plate. Linha azul, com splitter plate.

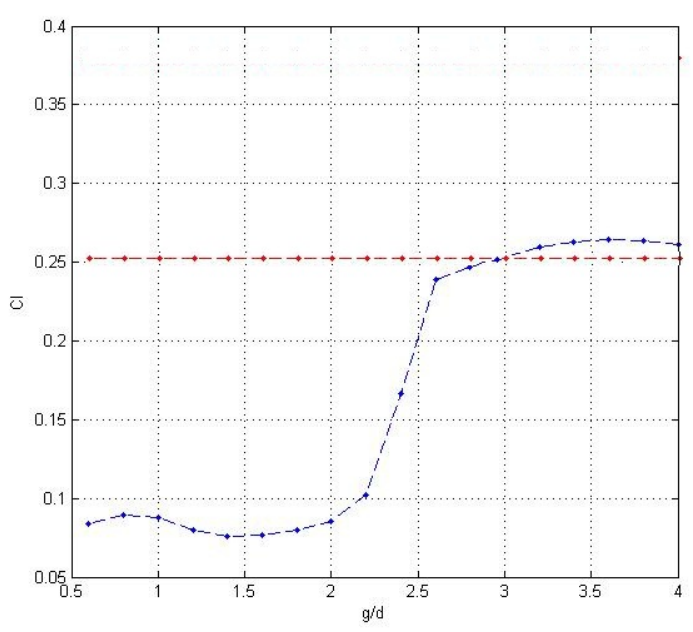

Figura 3.12: Máxima amplitude de $C_{l}$ versus $g / d$ para $R e=80$ e $l / d=0,5$. Linha vermelha, valores para o escoamento do cilindro sem splitter plate. Linha azul, com splitter plate.

ficiente para valor de $G A P$ de $g / d=1$, 4 . Após o ponto de mínimo, a curva foi recuperando o valor do coeficiente para o cilindro isolado gradativamente. Na região de $0,6 \leq g / d \leq 1,0$ ocorreu a formação de um máximo local, não mantendo a curva em comportamento decrescente. Esta região também foi encontrada por Igarashi (1982) em seus experimentos e está detalhada na revisão bibliográfica deste trabalho.

As figuras a seguir apresentam os resultados análogos ao anterior, exceto pelo comprimento do splitter plate que foi alterado para $l / d=1,0$

Para comprimento de placa $l / d=1,0$, o ponto de menor arrasto médio 


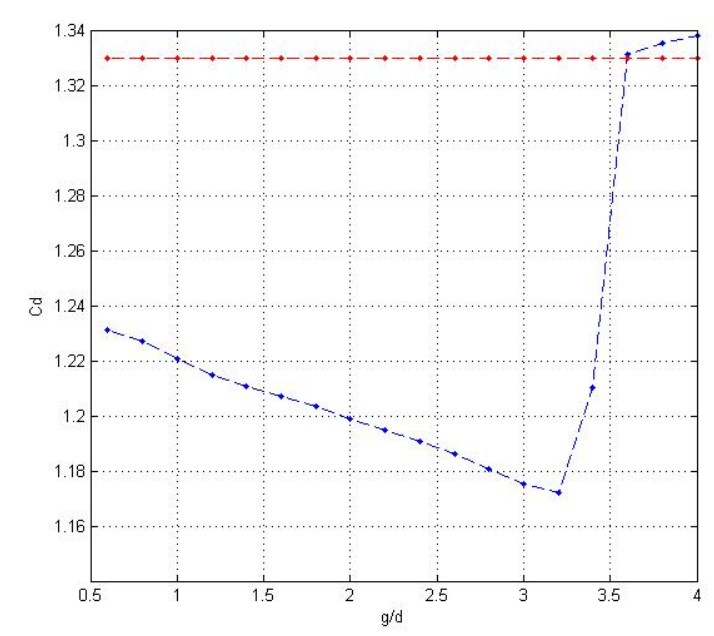

Figura 3.13: Coeficiente Médio de Arrasto $\overline{C_{d}}$ versus $g / d$, para $R e=80$ e $l / d=1,0$. Linha vermelha, valores para o escoamento do cilindro sem splitter plate. Linha azul, com splitter plate.

ocorreu para um $G A P$ de $g / d=3,2$, maior que para a placa com comprimento $l / d=0,5$. A redução do coeficiente de arrasto médio também foi maior, chegando a $12,0 \%$ em relação ao valor do coeficiente para o cilindro isolado. Quanto ao comportamento da curva, a recuperação para o valor do escoamento sem splitter plate foi mais abrupta em relação a placa de comprimento menor, entretanto, o comportamento totalmente decrescente até o valor de mínimo se manteve.

Para a sustentação, a redução da amplitude chegou a 96,0\%, mantendo o cilindro praticamente sem oscilação. O valor de GAP que ocorreu está redução, foi maior em relação ao comprimento $l / d=0,5$, porém foi o mesmo que o coeficiente de arrasto médio $(g / d=3,2)$. A curva apresentou uma pequena mudança na declividade, porém não apresentou máximo local como o caso anterior.

\subsubsection{2 $R e=100$}

De maneira análoga a seção 3.3.2.1, as figuras 3.15 a 3.18 apresentam os resultados para os comprimentos $l / d=0,5$ e 1,0 respectivamente.

Para comprimento de placa $l / d=0,5$, o ponto de menor arrasto médio ocorreu para um $G A P(g / d)$ de 2,0 com redução foi de $10,5 \%$ em relação ao valor do coeficiente de arrasto médio para um escoamento com cilindro sem splitter plate. Essa redução foi maior em relação à simulação com número de Reynolds igual a 80, considerando o mesmo comprimento de placa. Após esse valor de mínimo, o arrasto médio inicia um aumento mais abrupto até um valor intermediário, e depois segue subindo gradativamente, convergindo para o valor do 


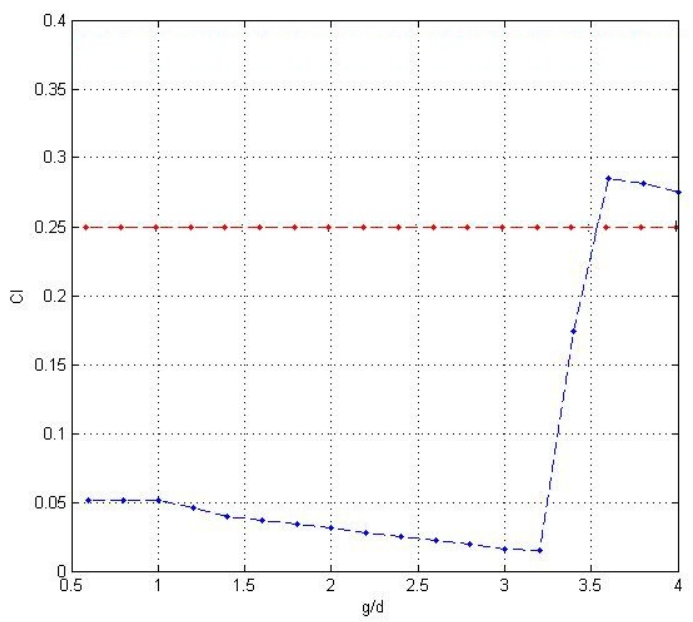

Figura 3.14: Máxima amplitude de $C_{l}$ versus $g / d$, para $R e=80$ e $l / d=1,0$. Linha vermelha, valores para o escoamento do cilindro sem splitter plate. Linha azul, com splitter plate.

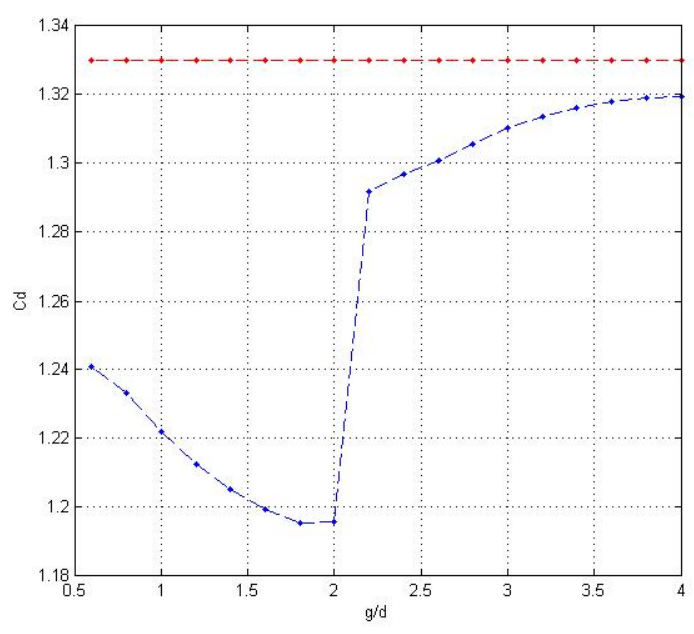

Figura 3.15: Coeficiente de Arrasto $\overline{C_{d}}$ versus $g / d$, para $R e=100$ e $l / d=0,5$. Linha vermelha, valores para o escoamento do cilindro sem splitter plate. Linha azul, com splitter plate.

cilindro isolado.

No caso da sustentação, houve uma redução de $73,8 \%$ da amplitude do coeficiente para valor de $G A P$ de $g / d=1,4$, sendo esta redução menor em relação as simulações com $R e=80$. Após o ponto de mínimo, a curva recuperou o valor do coeficiente para o cilindro isolado de maneira abrupta, sem uma região com inclinação mais suave, como ocorrido no coeficiente de arrasto médio. Na região de $0,6 \leq g / d \leq 1,0$ ocorreu a formação de um máximo local, não mantendo a curva em comportamento decrescente.

Para comprimento de placa $l / d=1,0$, o ponto de menor arrasto médio 


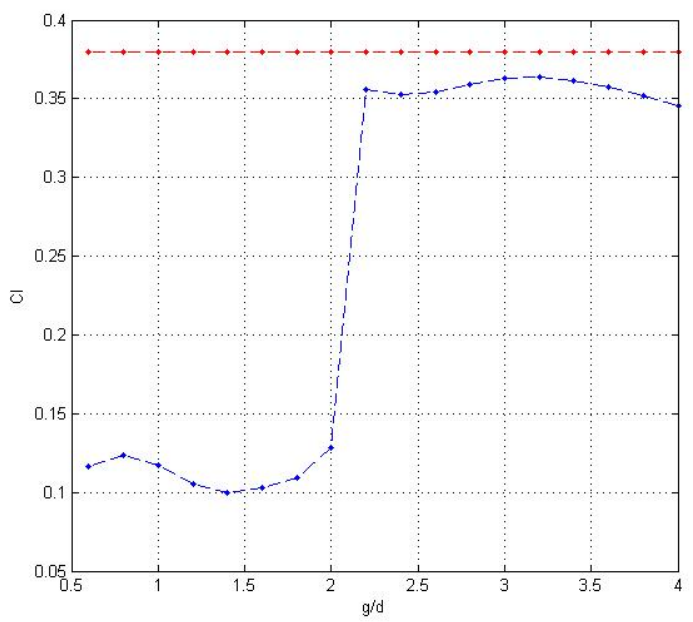

Figura 3.16: Máxima amplitude de $C_{l}$ para $R e=100$ e $l / d=0,5$. Linha vermelha, valores para o escoamento do cilindro sem splitter plate. Linha azul, com splitter plate.

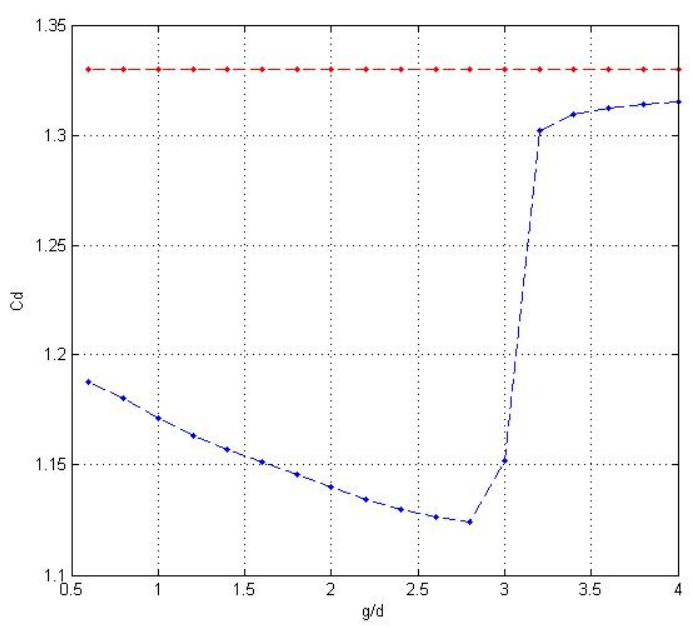

Figura 3.17: Coeficiente de Arrasto $\overline{C_{d}}$ versus $g / d$, para $R e=100$ e $l / d=1,0$. Linha vermelha, valores para o escoamento do cilindro sem splitter plate. Linha azul, com splitter plate.

ocorreu para um $G A P$ de $g / d=2,8$, menor em relação a placa com comprimento $l / d=0,5$ e também para a simulação com $R e=80$. A redução chegou a $15,8 \%$ em relação ao valor do coeficiente para o cilindro isolado. Quanto ao comportamento da curva, a recuperação para o valor do escoamento sem splitter plate foi abrupta com leve suavização após um afastamento maior do splitter plate.

Para a sustentação, a redução da amplitude chegou a 89,4\%, menor em relação a simulação com $R e=80$. O valor de $G A P$ que ocorreu esta redução foi maior em relação ao comprimento $l / d=0,5$, porém foi menor que o valor encontrado 


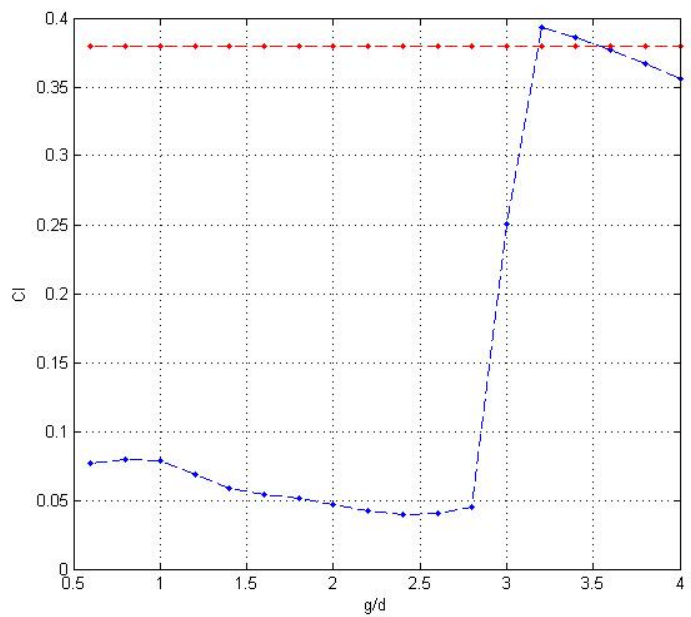

Figura 3.18: Máxima amplitude de $C_{l}$ para $R e=100$ e $l / d=1,0$. Linha vermelha, valores para o escoamento do cilindro sem splitter plate. Linha azul, com splitter plate.

para o coeficiente de arrasto médio $(g / d=3,4)$. Na região de $0,6 \leq g / d \leq 1,0$ ocorreu a formação de um máximo local, não mantendo a curva em comportamento decrescente. Vale ressaltar que a recuperação da amplitude de sustentação foi abrupta após o ponto de mínimo chegando a ultrapassar o valor da amplitude no caso de cilindro isolado.

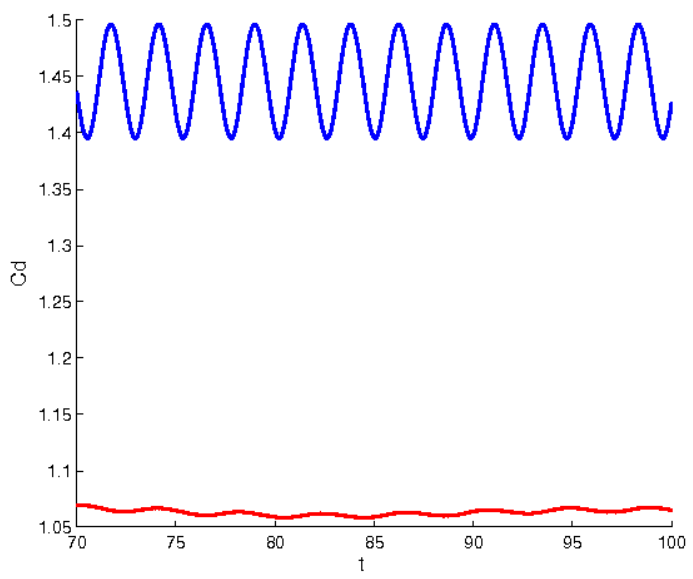

Figura 3.19: Média do Coeficiente de Arrasto $\overline{C_{d}}$ ao longo do tempo, para $R e=100$ e $l / d=1,0$. Linha azul, valores para o escoamento do cilindro sem splitter plate. Linha vermelha, com splitter plate na posição de $g / d$ otimizada.

\subsubsection{3 $R e=120$}

As figuras 3.21 a 3.24 apresentam os resultados para os comprimentos $l / d=$ 0,5 e 1,0 respectivamente. 


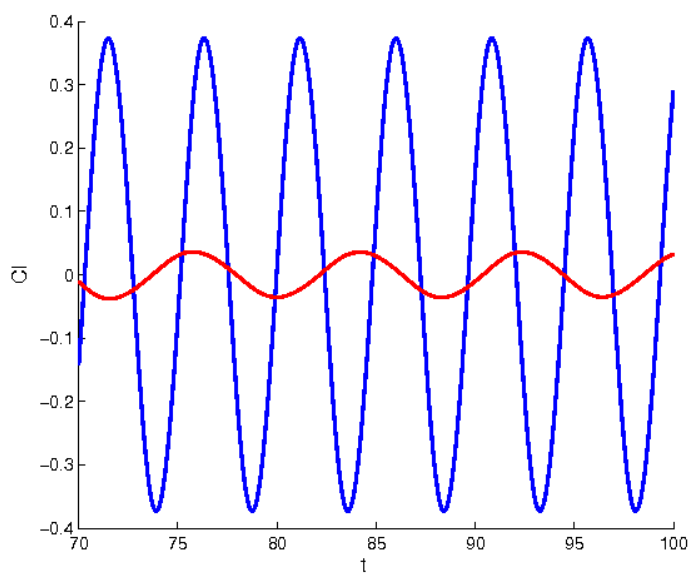

Figura 3.20: Máxima amplitude de $C_{l}$ ao longo do tempo, para $R e=100 \mathrm{e}$ $l / d=1.0$. Linha azul, valores para o escoamento do cilindro sem splitter plate. Linha vermelha, com splitter plate na posição de $g / d$ otimizada.

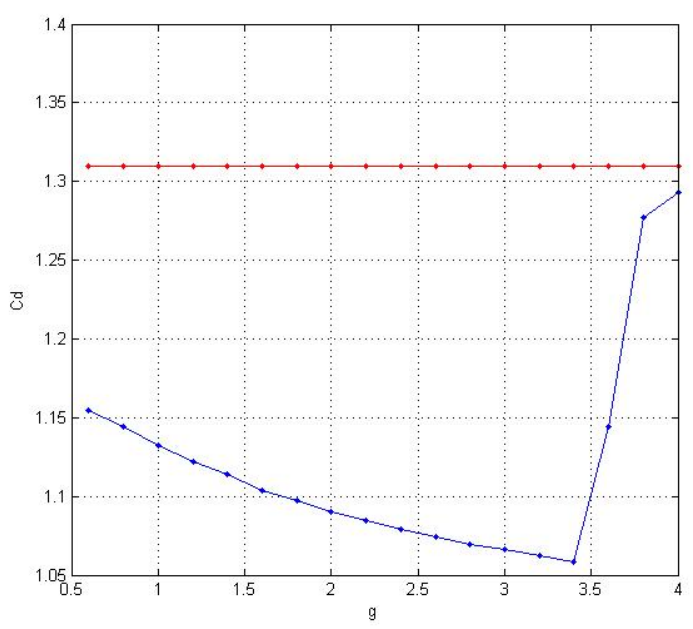

Figura 3.21: Coeficiente de Arrasto $\overline{C_{d}}$ versus $g / d$, para $R e=120$ e $l / d=0,5$. Linha vermelha, valores para o escoamento do cilindro sem splitter plate. Linha azul, com splitter plate.

Para comprimento de placa $l / d=0,5$, o ponto de menor arrasto médio ocorreu para um $G A P(g / d)$ de 1,8 com redução foi de $11,2 \%$ em relação ao valor do coeficiente de arrasto médio para um escoamento com cilindro sem splitter plate. Essa redução foi maior em relação à simulação com número de Reynolds igual a 80 e 100, considerando o mesmo comprimento de placa. Após esse valor de mínimo, o arrasto médio inicia um aumento mais abrupto até um valor intermediário, e depois segue subindo gradativamente, convergindo para o valor do cilindro isolado.

No caso da sustentação, houve uma redução de 73,0\% da amplitude do coeficiente para valor de $G A P$ de $g / d=1,4$, sendo esta redução menor em relação 


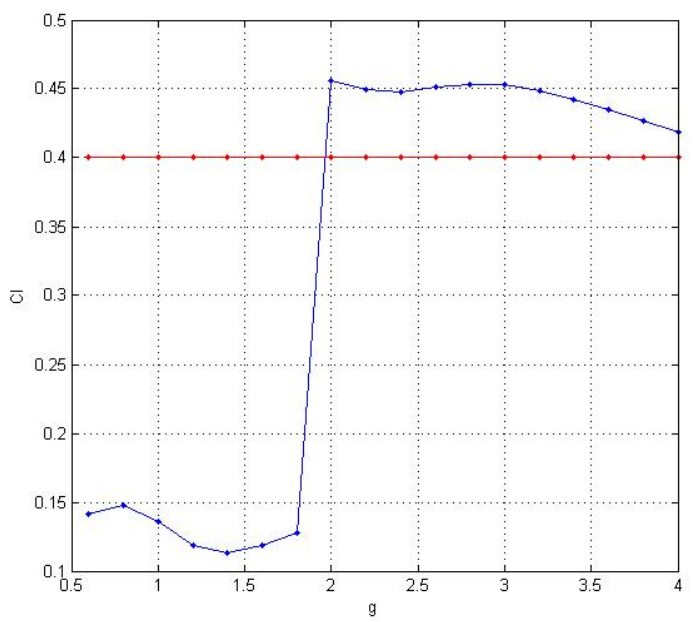

Figura 3.22: Máxima amplitude de $C_{l}$ para $R e=120$ e $l / d=0,5$. Linha vermelha, valores para o escoamento do cilindro sem splitter plate. Linha azul, com splitter plate.

as simulações com $R e=80$ e $R e=100$. Após o ponto de mínimo, a curva recuperou o valor do coeficiente de maneira abrupta chegando a ultrapassar o valor da amplitude no caso de cilindro isolado. Na região de $0,6 \leq g / d \leq 1,0$ ocorreu a formação de um máximo local, não mantendo a curva em comportamento decrescente.

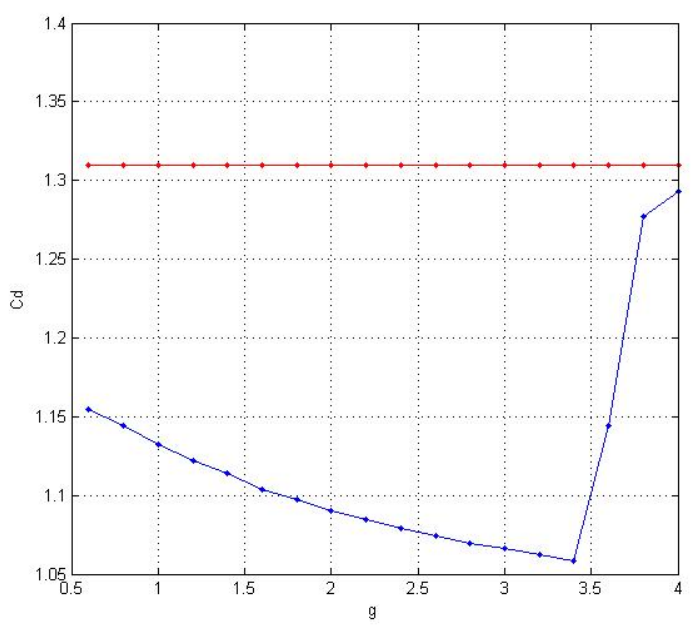

Figura 3.23: Coeficiente de Arrasto $\overline{C_{d}}$ versus $g / d$, para $R e=120$ e $l / d=1,0$. Linha vermelha, valores para o escoamento do cilindro sem splitter plate. Linha azul, com splitter plate.

Para comprimento de placa $l / d=1,0$, o ponto de menor arrasto médio ocorreu para um $G A P$ de $g / d=3,4$, maior em relação a placa com comprimento $l / d=0,5$ e também para a simulação com $R e=100$. A redução chegou a $21,0 \%$ em relação ao valor do coeficiente para o cilindro isolado. Quanto ao 
comportamento da curva, a recuperação para o valor do escoamento sem splitter plate foi abrupta com leve suavização após um afastamento maior do splitter plate.

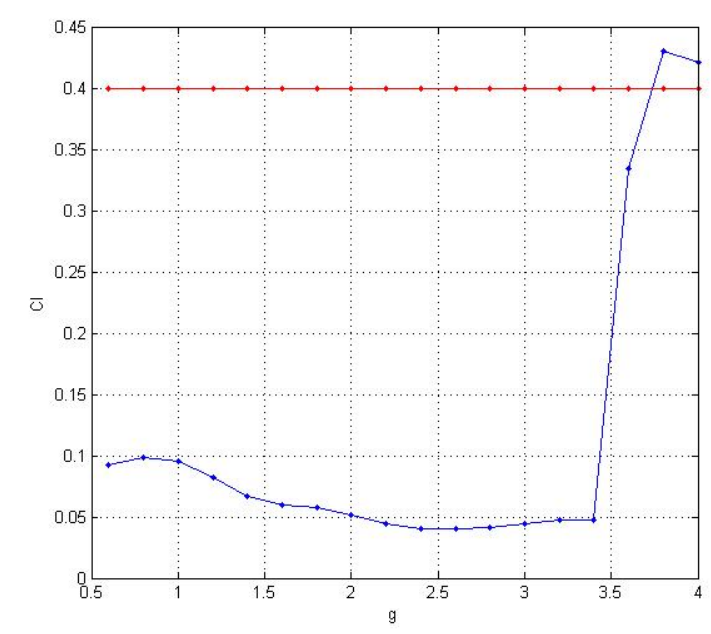

Figura 3.24: Máxima amplitude de $C_{l}$ para $R e=120$ e $l / d=1,0$. Linha vermelha, valores para o escoamento do cilindro sem splitter plate. Linha azul, com splitter plate.

Para a sustentação, a redução da amplitude chegou a 87,2\%, menor em relação a simulação com $R e=100$. O valor de GAP que ocorreu esta redução foi maior em relação ao comprimento $l / d=0,5$, porém foi menor que o valor encontrado para o coeficiente de arrasto médio $(g / d=2,6)$. Na região de $0,6 \leq g / d \leq 1,0$ ocorreu a formação de um máximo local, não mantendo a curva em comportamento decrescente. Vale ressaltar que a recuperação da amplitude de sustentação foi abrupta após o ponto de mínimo chegando a ultrapassar o valor da amplitude no caso de cilindro isolado.

\subsubsection{4 $R e=140$}

As figuras 3.25 a 3.28 apresentam os resultados para os comprimentos $l / d=$ 0,5 e 1,0 respectivamente.

Para comprimento de placa $l / d=0,5$, o ponto de menor arrasto médio ocorreu para um $G A P(g / d)$ de 2,0 com redução foi de $13,5 \%$ em relação ao valor do coeficiente de arrasto médio para um escoamento com cilindro sem splitter plate. Essa redução foi maior em relação à simulação com aos números de Reynolds simulados, considerando o mesmo comprimento de placa. Após esse valor de mínimo, o arrasto médio inicia um aumento mais abrupto até um valor intermediário, e depois segue subindo gradativamente, convergindo para o valor do 


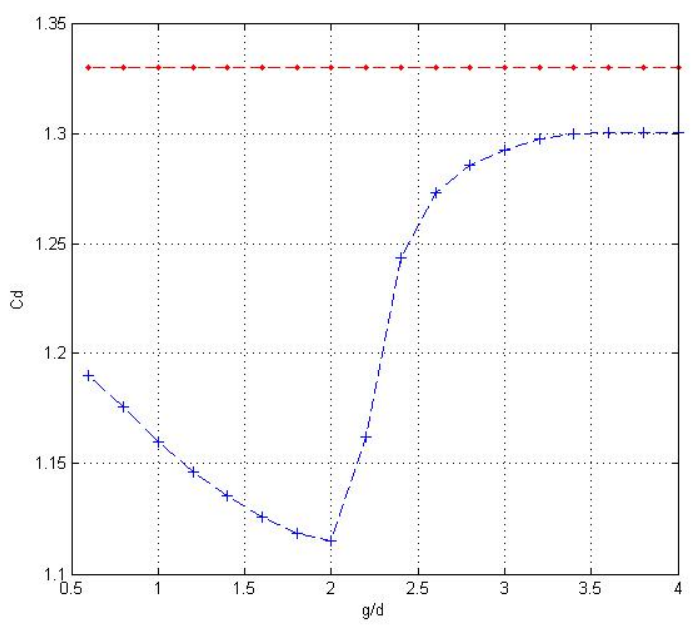

Figura 3.25: Coeficiente de Arrasto $\overline{C_{d}}$ versus $g / d$, para $R e=140$ e $l / d=0,5$. Linha vermelha, valores para o escoamento do cilindro sem splitter plate. Linha azul, com splitter plate.

cilindro isolado.

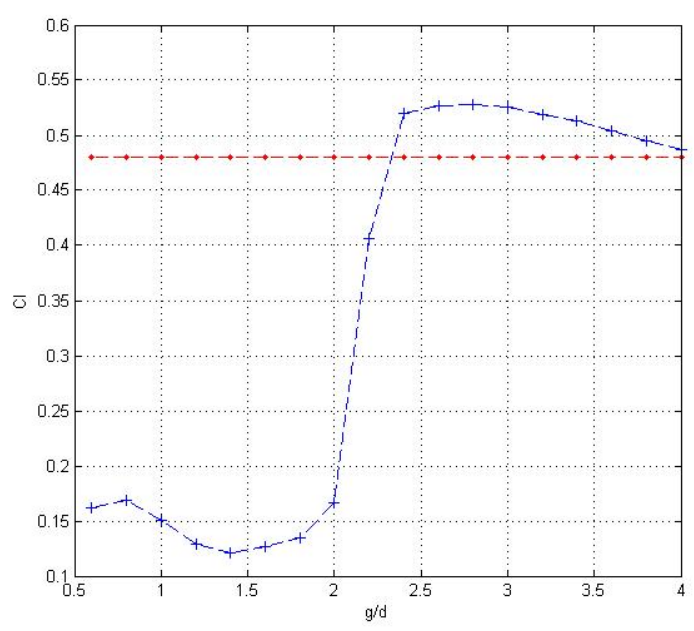

Figura 3.26: Máxima amplitude de $C_{l}$ para $R e=140$ e $l / d=0,5$. Linha vermelha, valores para o escoamento do cilindro sem splitter plate. Linha azul, com splitter plate.

No caso da sustentação, houve uma redução de 74,7\% da amplitude do coeficiente para valor de $G A P$ de $g / d=1,4$, sendo esta redução um pouco maior em relação as simulações com $R e=100 e 120$. Após o ponto de mínimo, a curva iniciou um aumento suave até o valor de $g / d=2,0$ e após esse valor, subiu de maneira abrupta chegando a ultrapassar o valor da amplitude no caso de cilindro isolado. Na região de $0,6 \leq g / d \leq 1,0$ ocorreu a formação de um máximo local, não mantendo a curva em comportamento decrescente.

Para comprimento de placa $l / d=1,0$, o ponto de menor arrasto médio 


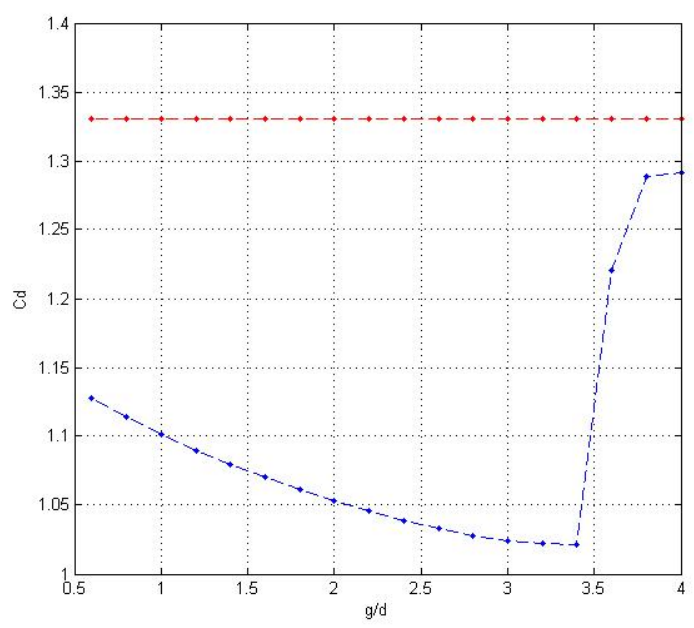

Figura 3.27: Coeficiente de Arrasto $\overline{C_{d}}$ versus $g / d$, para $R e=140$ e $l / d=1,0$. Linha vermelha, valores para o escoamento do cilindro sem splitter plate. Linha azul, com splitter plate.

ocorreu para um $G A P$ de $g / d=3,4$, maior em relação a placa com comprimento $l / d=0,5$ e também para a simulação com $R e=120$. A redução chegou a 23,3\% em relação ao valor do coeficiente para o cilindro isolado. Quanto ao comportamento da curva, a recuperação para o valor do escoamento sem splitter plate foi abrupta com leve suavização após um afastamento maior do splitter plate.

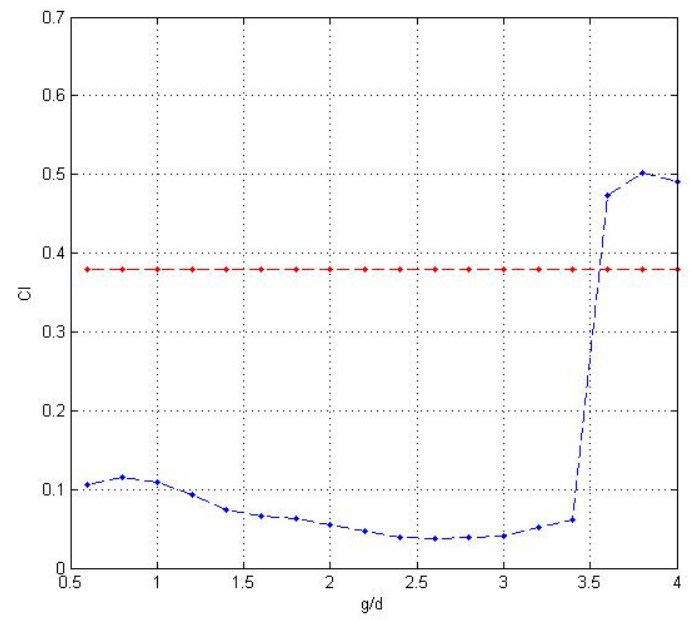

Figura 3.28: Máxima amplitude de $C_{l}$ para $R e=140$ e $l / d=1,0$. Linha vermelha, valores para o escoamento do cilindro sem splitter plate. Linha azul, com splitter plate.

Para a sustentação, a redução da amplitude chegou a 92,1\%, maior em relação a simulação com $R e=100$ e $R e=120$. O valor de $G A P$ que ocorreu esta redução foi maior em relação ao comprimento $l / d=0,5$, porém foi menor que o 
valor encontrado para o coeficiente de arrasto médio $(g / d=2,6)$. Na região de $0,6<g / d<1,0$ ocorreu a formação de um máximo local, não mantendo a curva em comportamento decrescente. Vale ressaltar que a recuperação da amplitude de sustentação foi abrupta após o ponto de mínimo chegando a ultrapassar o valor da amplitude no caso de cilindro isolado.

A figura 3.29 mostra os quadros temporais do escoamento ao longo de um período, apresentando a movimentação da esteira de vórtices após o bordo de fuga do splitter plate. Vale ressaltar que essa figura foi gerada no ponto $g / d=3,4$, ou seja, no ponto de mínimo arrasto médio e amplitude de sustentação.
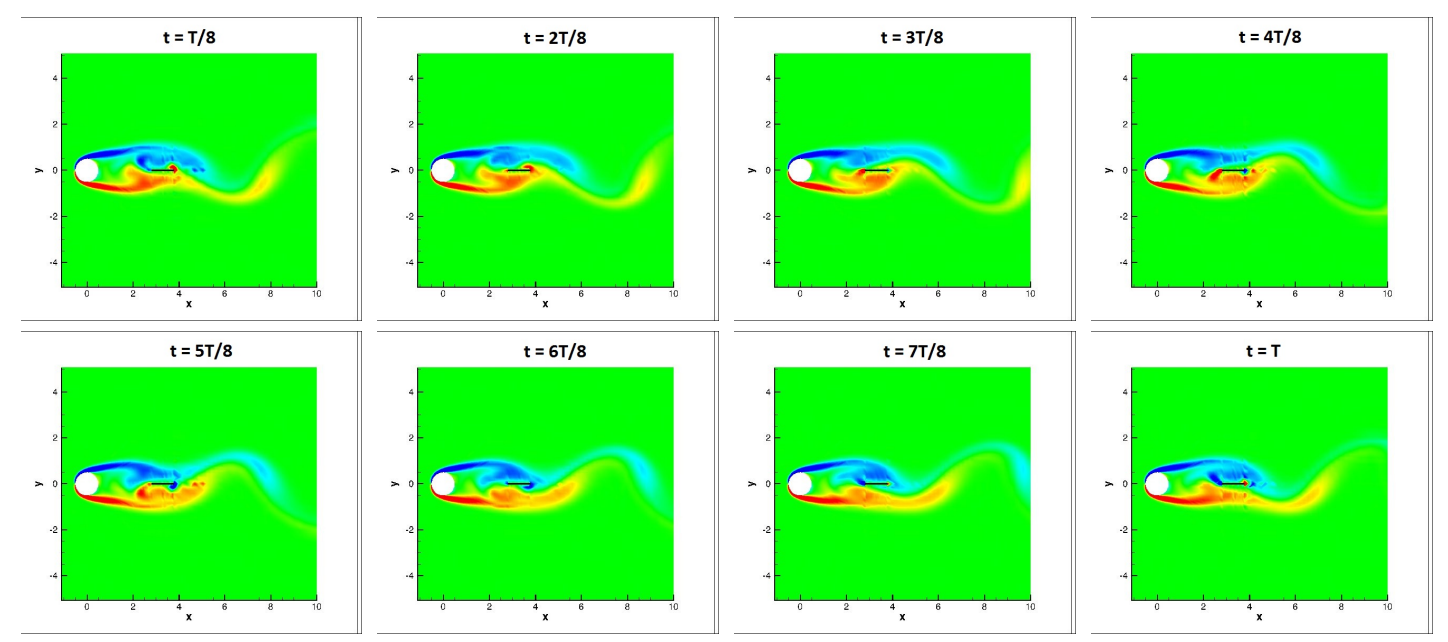

Figura 3.29: Quadros temporais da movimentação da vorticidade, $l / d=1,0$ e $g / d=3,4(R e=140)$

De maneira a visualizar o escoamento após a passagem do ponto de mínimo, a figura 3.30 apresenta uma visualização do escoamento quando o splitter plate ultrapassa do valor da GAP crítico $(g / d=4,0)$.
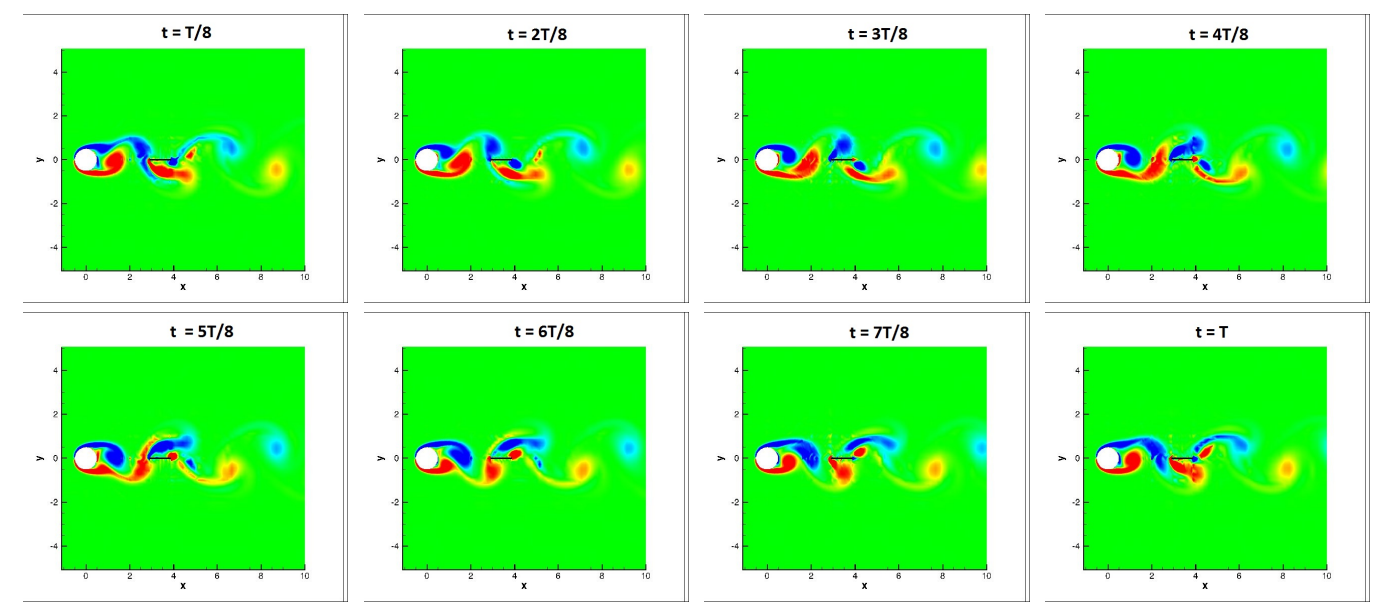

Figura 3.30: Quadros temporais da movimentação da vorticidade, $l / d=1,0$ e $g / d=4,0(R e=120)$. 
É possível observar a interação entre as esteiras de vórtices nas proximidades do cilindro, como se não houvesse nenhum splitter plate. O vórtice então é cortado pela placa gerando pequenas componentes de vorticidade que se juntam ao vórtice anterior e ao vórtice posterior.

Finalizando os resultados para o arrasto e sustentação, as tabelas 3.3 e 3.4 apresentam um resumo com todos os resultados obtidos:

Tabela 3.3: Resumo dos resultados - Média Temporal do Coeficiente de Arrasto

\begin{tabular}{cccc}
\hline Reynolds & l/d & g/d (mínimo) & Valor (Redução) \\
\hline 80 & 0,5 & 2,0 & $6,3 \%$ \\
80 & 1,0 & 3,2 & $12,0 \%$ \\
100 & 0,5 & 2,0 & $10,5 \%$ \\
100 & 1,0 & 2,8 & $15,8 \%$ \\
120 & 0,5 & 1,8 & $11,2 \%$ \\
120 & 1,0 & 3,4 & $21,0 \%$ \\
140 & 0,5 & 2,0 & $13,5 \%$ \\
140 & 1,0 & 3,4 & $23,3 \%$
\end{tabular}

Tabela 3.4: Resumo dos resultados - Amplitude do Coeficiente de Sustentação

\begin{tabular}{cccc}
\hline Reynolds & l/d & g/d (mínimo) & Valor (Redução) \\
\hline 80 & 0,5 & 1,4 & $80,0 \%$ \\
80 & 1,0 & 3,2 & $96,0 \%$ \\
100 & 0,5 & 1,4 & $73,8 \%$ \\
100 & 1,0 & 3,4 & $89,4 \%$ \\
120 & 0,5 & 1,4 & $73,0 \%$ \\
120 & 1,0 & 2,6 & $87,2 \%$ \\
140 & 0,5 & 1,4 & $74,7 \%$ \\
140 & 1,0 & 2,6 & $92,1 \%$
\end{tabular}




\subsubsection{Resultados Complementares}

As seções anteriores apresentaram os resultados para os coeficientes de arrasto e sustentação, ressaltando que a dedução da solução adjunta neste trabalho focará na redução da média do coeficiente de arrasto.

Entretanto, vale ressaltar que existem outros 3 parâmetros que são de interesse no estudo de escoamento ao redor de corpos rombudos. Estes parâmetros, juntamente com a amplitude da sustentação, também podem ser usados como medida de mérito.

O primeiro dos parâmetros é o número de Strouhal, que foi definido na seção 1.2 e está diretamente ligado a frequência de desprendimento de vórtices. A figuras 3.31 a 3.34 apresentam o comportamento do número de Strouhal para valores de $R e=80$ a $R e=140$ com o comprimento de placa $l / d=0,5$.

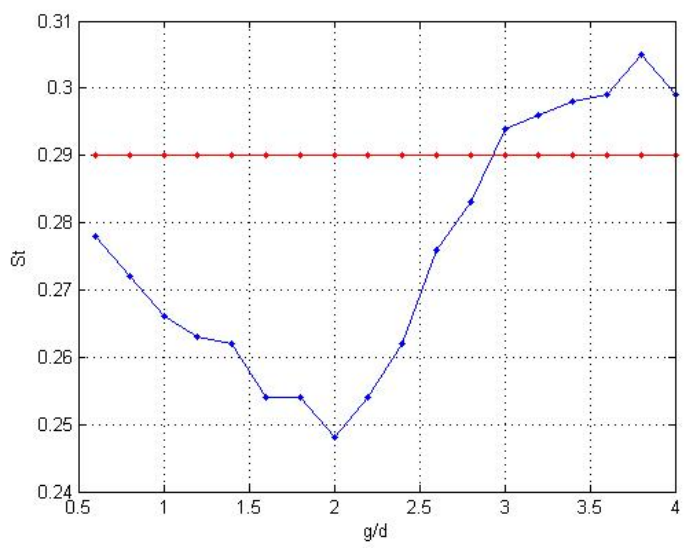

Figura 3.31: Número de Strouhal $S t$ versus $g / d$, para $l / d=0,5$ e $R e=80$. Linha Vermelha, sem splitter plate; Linha azul, com splitter plate.

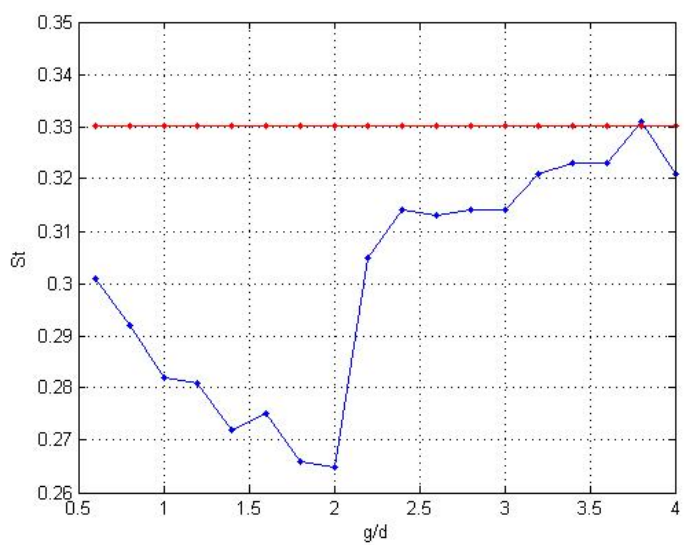

Figura 3.32: Número de Strouhal $S t$ versus $g / d$, para $l / d=0,5$ e $R e=100$. Linha Vermelha, sem splitter plate; Linha azul, com splitter plate. 
O comportamento do número de Strouhal retrata a diminuição da frequência de vibração do cilindro com a presença do splitter plate. Exceto pelo ponto $g / d=2,8$ para $R e=120$ que ficou fora do comportamento visto em Igarashi (1982). Observa-se que as curvas do gráfico diminuem até se chegar no ponto de mínimo e após esse ponto, o adimensional aumenta de valor, ficando próximo do valor para o caso do cilindro isolado.

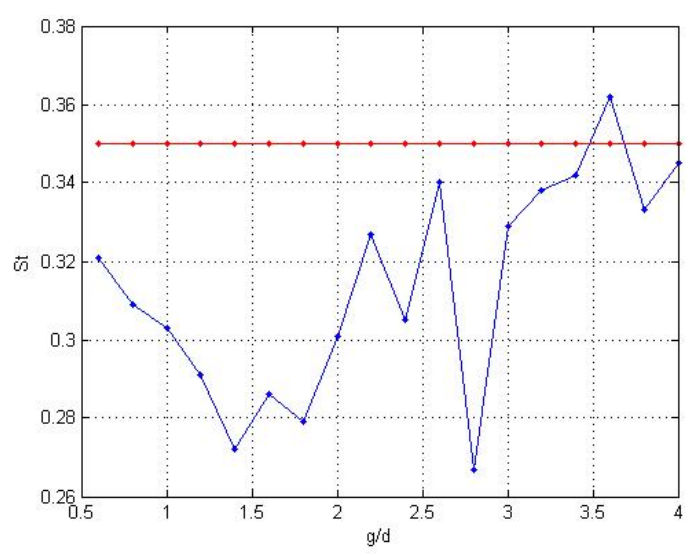

Figura 3.33: Número de Strouhal $S t$ versus $g / d$, para $l / d=0,5$ e $R e=120$. Linha Vermelha, sem splitter plate; Linha azul, com splitter plate.

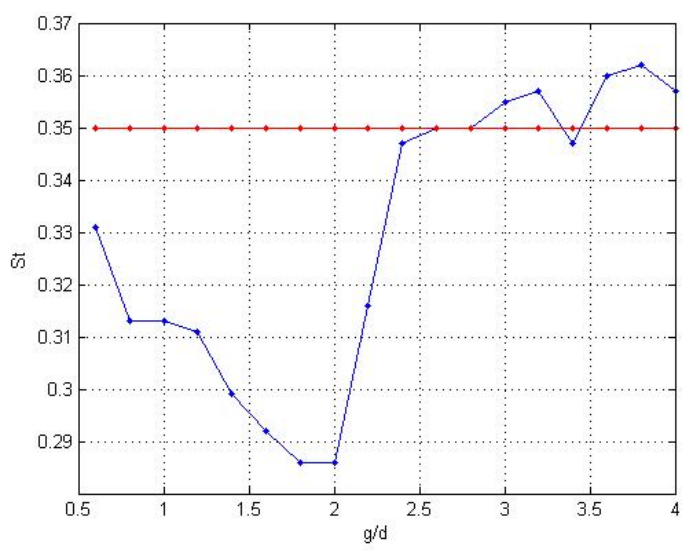

Figura 3.34: Número de Strouhal $S t$ versus $g / d$, para $l / d=0,5$ e $R e=140$. Linha Vermelha, sem splitter plate; Linha azul, com splitter plate.

Para $R e=80$, a redução máxima foi de $13,8 \%$ em relação ao caso do cilindro sem splitter plate. Para os valores de $R e=100$, a redução foi de 19,6\% em relação ao cilindro isolado e para número de Reynolds igual a 120 e 140, as reduções máximas foram de 18,1 (Desconsiderando o ponto $g / d=2,8$ ) e $18,5 \%$ respectivamente.

No caso de $l / d=1,0$, o comportamento das curvas não apresentou nenhum ponto destoante em relação as apresentadas por Igarashi (1984). Para $R e=80$, 


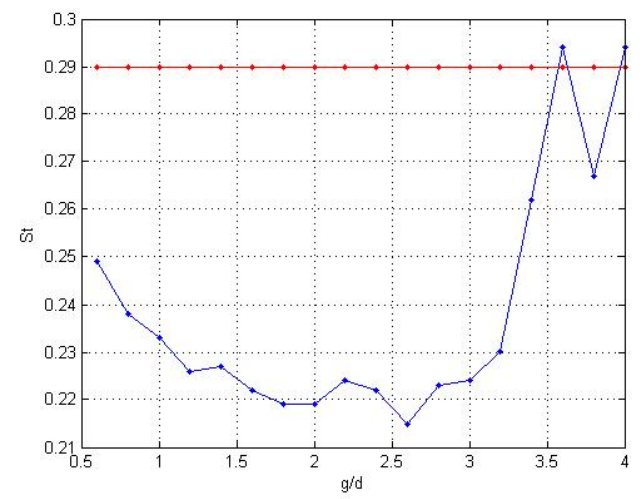

Figura 3.35: Número de Strouhal $S t$ versus $g / d$, para $l / d=1,0$ e $R e=80$. Linha Vermelha, sem splitter plate; Linha azul, com splitter plate.

a redução máxima foi de 25,8\% em relação ao caso sem splitter plate. Para os valores de $R e=100,120$ e 140, as reduções foram de 28,7,35, 7 e 37, 1\% respectivamente.

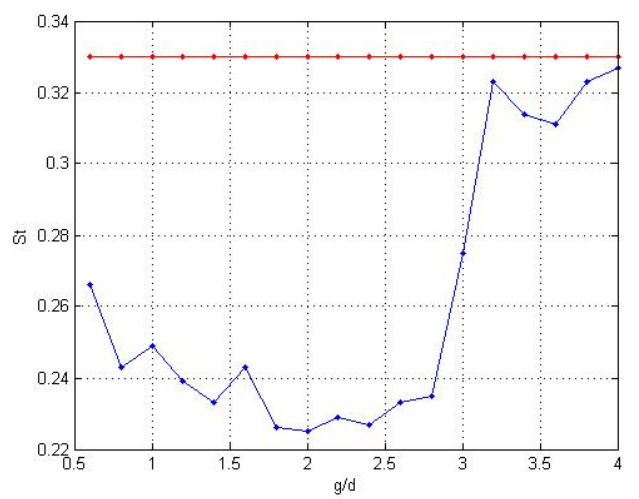

Figura 3.36: Número de Strouhal $S t$ versus $g / d$, para $l / d=1,0$ e $R e=100$. Linha Vermelha, sem splitter plate; Linha azul, com splitter plate.

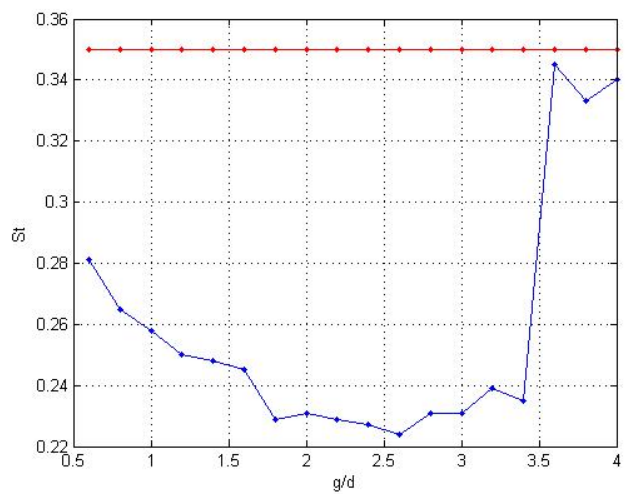

Figura 3.37: Número de Strouhal $S t$ versus $g / d$, para $l / d=1,0$ e $R e=120$. Linha Vermelha, sem splitter plate; Linha azul, com splitter plate. 


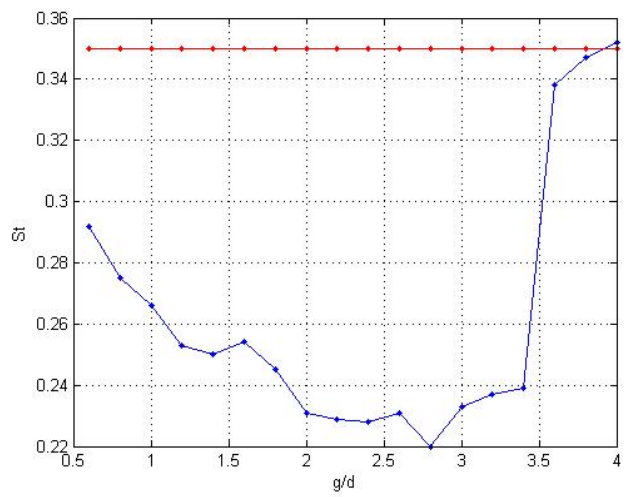

Figura 3.38: Número de Strouhal $S t$ versus $g / d$, para $l / d=1,0$ e $R e=140$. Linha Vermelha, sem splitter plate; Linha azul, com splitter plate.

É importante relembrar que o número de Strouhal não é uma propriedade física obtida diretamente do escoamento, pois ela é calculada a partir da frequência de vibração do sistema, obtida através dos gráficos de oscilação das força de arrasto, por exemplo.

De maneira resumida, a tabela 3.5 apresenta todos os resultados obtidos:

Tabela 3.5: Resumo dos resultados - Número de Strouhal

\begin{tabular}{cccc}
\hline Reynolds & l/d & g/d (mínimo) & Valor (Redução) \\
\hline 80 & 0,5 & 2,0 & $13,8 \%$ \\
80 & 1,0 & 2,6 & $25,8 \%$ \\
100 & 0,5 & 2,0 & $19,6 \%$ \\
100 & 1,0 & 2,0 & $28,7 \%$ \\
120 & 0,5 & 1,4 & $18,1 \%$ \\
120 & 1,0 & 2,6 & $35,7 \%$ \\
140 & 0,5 & 2,0 & $18,5 \%$ \\
140 & 1,0 & 2,6 & $37,1 \%$
\end{tabular}

O segundo parâmetro é o comprimento de formação $l_{f}$. As figuras 3.39 a 3.46 apresentam os efeitos do posicionamento do splitter plate no comprimento de formação dos vórtices, para dois comprimentos de placa: $l / d=0,5$ e $l / d=1,0$, respectivamente.

O comprimento de formação possui uma característica diferente dos demais parâmetros, pois o mesmo cresce até um valor máximo e após esse ponto ele 


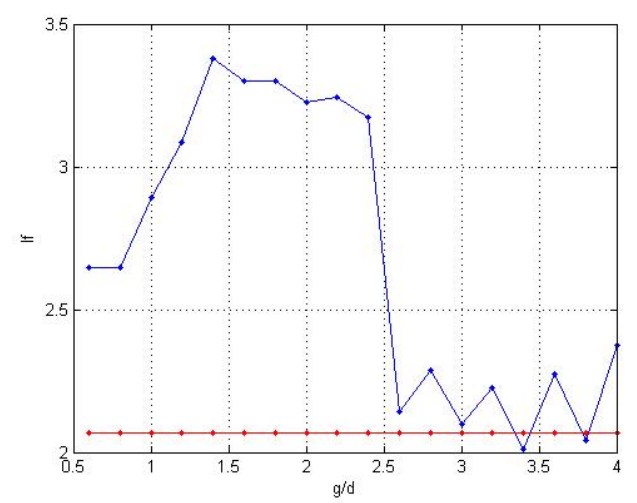

Figura 3.39: Comprimento de Formação $l_{f}$ versus $g / d$, para $l / d=0,5 \mathrm{e}$ $R e=80$. Linha Vermelha, sem splitter plate; Linha azul, com splitter plate.

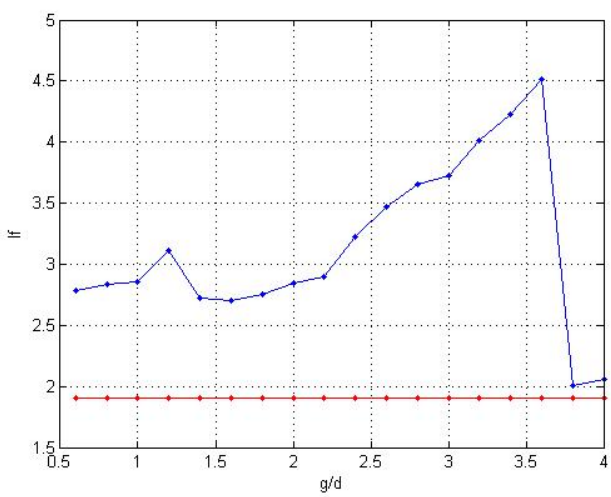

Figura 3.40: Comprimento de Formação $l_{f}$ versus $g / d$, para $l / d=0,5 \mathrm{e}$ $R e=100$. Linha Vermelha, sem splitter plate; Linha azul, com splitter plate.

diminui até o valor do caso do cilindro sem splitter plate. Vale relembrar que o comprimento de formação é a distância da iteração dos vórtices em relação ao cilindro e que a função do splitter plate é justamente afastar os vórtices do corpo rombudo.

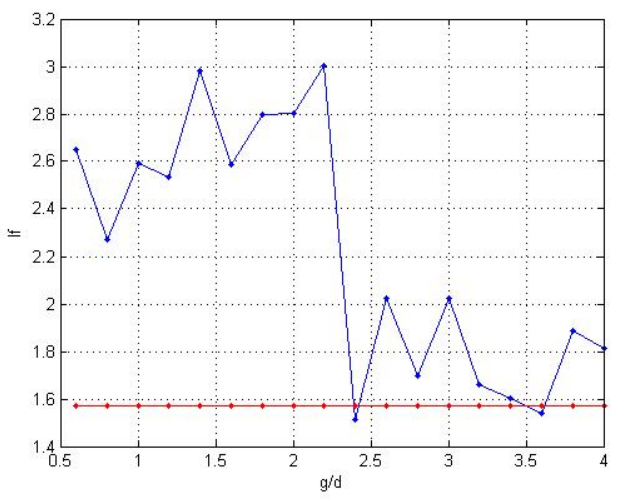

Figura 3.41: Comprimento de Formação $l_{f}$ versus $g / d$, para $l / d=0,5 \mathrm{e}$ $R e=120$. Linha Vermelha, sem splitter plate; Linha azul, com splitter plate. 
No caso de $l / d=0,5$, o comportamento das curvas não apresentou nenhum ponto destoante em relação as apresentadas por Igarashi (1984). Para $R e=80$, houve uma oscilação a partir da queda abrupta em $g / d=2,6$, chegando a um distanciamento máximo de 61, $9 \%$ do comprimento de formação para o escoamento sem splitter plate. Para os valores de $R e=100,120$ e 140, os distanciamentos foram de $57,8,87,5$ e $94,8 \%$ respectivamente.

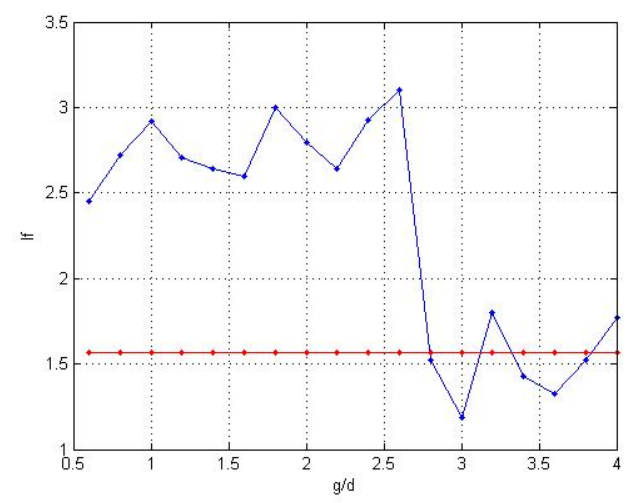

Figura 3.42: Comprimento de Formação $l_{f}$ versus $g / d$, para $l / d=0,5 \mathrm{e}$ $R e=140$. Linha Vermelha, sem splitter plate; Linha azul, com splitter plate.

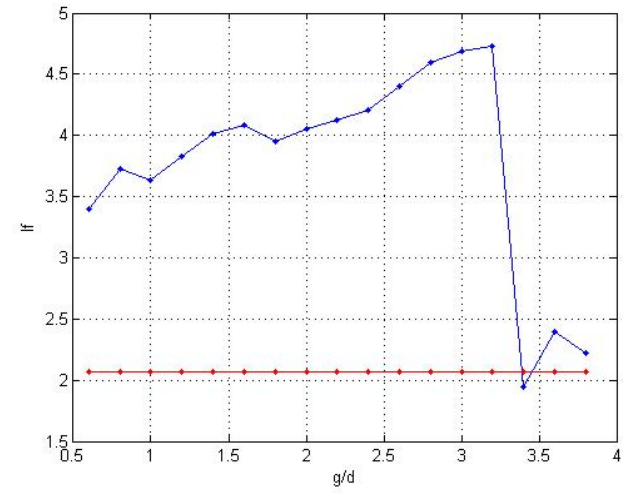

Figura 3.43: Comprimento de Formação $l_{f}$ versus $g / d$, para $l / d=1,0 \mathrm{e}$ $R e=80$. Linha Vermelha, sem splitter plate; Linha azul, com splitter plate.

Para $l / d=1,0$, o comportamento das curvas se manteve semelhante ao que foi visto em (IGARASHI, 1984). Para $R e=80$, o distanciamento máximo foi de $137,5 \%$ do comprimento de formação para o escoamento sem splitter plate. Para o valor de $R e=100$, o distanciamento foi de $125,0 \%$.

No caso de número de Reynolds igual a 120, o comprimento de formação foi 206, 7\% maior que o comprimento de formação no caso do cilindro isolado. E por fim para $R e=140$, o distanciamento foi de $187,5 \%$.

O comprimento de formação é um parâmetro muito estudado principalmente 


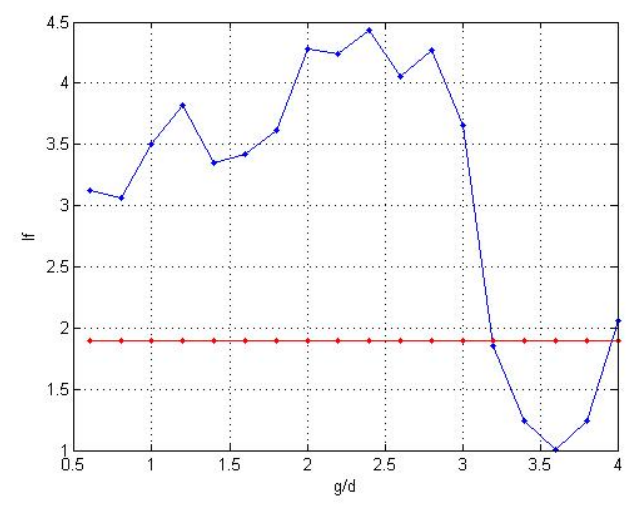

Figura 3.44: Comprimento de Formação $l_{f}$ versus $g / d$, para $l / d=1,0$ e $R e=100$. Linha Vermelha, sem splitter plate; Linha azul, com splitter plate.

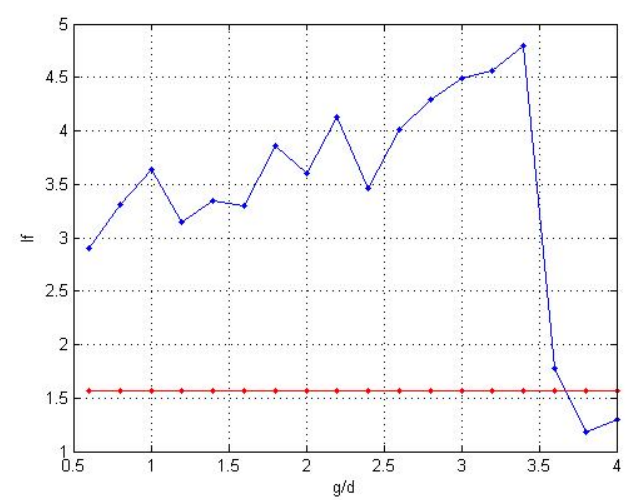

Figura 3.45: Comprimento de Formação $l_{f}$ versus $g / d$, para $l / d=1,0$ e $R e=120$. Linha Vermelha, sem splitter plate; Linha azul, com splitter plate.

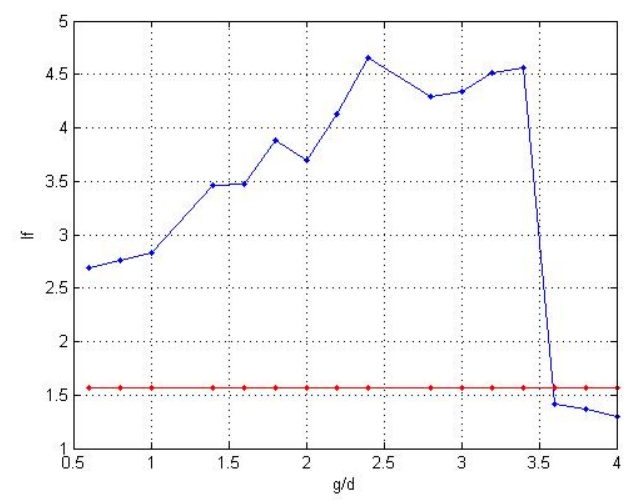

Figura 3.46: Comprimento de Formação $l_{f}$ versus $g / d$, para $l / d=1,0$ e $R e=140$. Linha Vermelha, sem splitter plate; Linha azul, com splitter plate.

nos casos que envolvem escoamento ao redor de dois cilindros na posição de "tandem". Meneghini (2001) apresentou alguns resultados interessantes da variação do comprimento de formação neste tipo de escoamento. Ele observou que a presença do segundo cilindro proporciona um deslocamento da camada de vórtice 
para a jusante do mesmo, aumentando o comprimento de formação em relação ao cilindro a montante.

Tanto Meneghini (2001) e Carmo (2005) mencionaram que o espaçamento entre os cilindros possuia um valor crítico, e que após essa distância, a presença do segundo cilindro não era suficiente para impedir a formação de vórtices entre os dois cilindros. Neste aspecto, o fenômeno é análogo ao caso do splitter plate deste trabalho.

A diferença relevante dos dois estudos é que no caso visto por Meneghini (2001) e Carmo (2005) ocorre um fenômeno conhecido como inversão de arrasto, onde o sentido do vetor de arrasto do cilindro a jusante fica oposto a direção do escoamento. No presente trabalho, ocorre apenas a redução da média temporal do coeficiente, não chegando a inverter o seu sentido.

Vale ressaltar também que as referências realizaram simulações tridimensionais e determinaram a variação do comprimento de formação para faixas de número de Reynolds acima do valor de 140, utilizado como um valor que diminui os efeitos de tridimensionalidade.

De maneira resumida, a tabela 3.6 apresenta todos os resultados obtidos:

Tabela 3.6: Resumo dos resultados - Comprimento de Formação

\begin{tabular}{cccc}
\hline Reynolds & $\mathbf{l} / \mathbf{d}$ & $\mathbf{g} / \mathbf{d}$ (máximo) & Valor (Aumento) \\
\hline 80 & 0,5 & 1,4 & $61,9 \%$ \\
80 & 1,0 & 3,4 & $37,5 \%$ \\
100 & 0,5 & 3,6 & $57,8 \%$ \\
100 & 1,0 & 2,4 & $25,0 \%$ \\
120 & 0,5 & 2,6 & $87,5 \%$ \\
120 & 1,0 & 3,4 & $106,7 \%$ \\
140 & 0,5 & 3,4 & $94,8 \%$ \\
140 & 1,0 & 2,4 & $87,5 \%$
\end{tabular}

Encerrando os resultados complementares, o terceiro parâmetro que também é de interesse para o estudo é o coeficiente médio de pressão na base do cilindro, $\overline{-C_{p b}}$.

A pressão é uma variável medida experimentalmente e há estudos que chegam a mapear toda a região do cilindro. Por ter essa medição experimental facilitada, 
os trabalhos da metade do século XX foram mais focados neste parâmetro (IGARASHI, 1982).

As figuras 3.47 e 3.54 apresentam o comportamento do coeficiente com a variação do GAP entre o splitter plate e o cilindro. Analogamente aos casos anteriores, os resultados são para os comprimentos de placa $l / d=0,5$ e $l / d=1,0$.

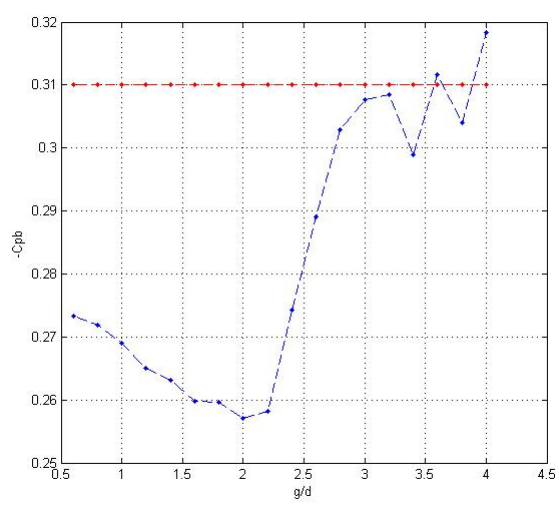

Figura 3.47: Coeficiente Médio de Pressão na Base do Cilindro $\overline{-C_{p b}}$ versus $g / d$, para $l / d=0,5$ e $R e=80$. Linha Vermelha, sem splitter plate; Linha azul, com splitter plate.

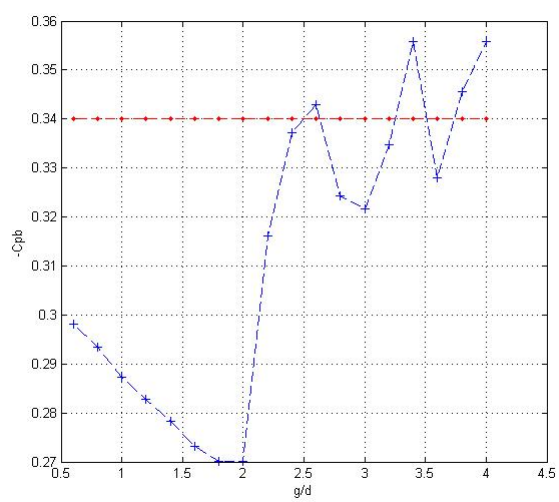

Figura 3.48: Coeficiente Médio de Pressão na Base do Cilindro $\overline{-C_{p b}}$ versus $g / d$, para $l / d=0,5$ e $R e=100$. Linha Vermelha, sem splitter plate; Linha azul, com splitter plate.

No caso de $l / d=0,5$, o comportamento das curvas não apresentou nenhum ponto destoante em relação as apresentadas por Igarashi (1982).

Para $R e=80$, a redução foi de 16,1\% em relação ao escoamento sem splitter plate. No caso $R e=100$, a redução foi de $25,9 \%$ em relação ao coeficiente de pressão medido no escoamento com o cilindro isolado. Já para 120 e 140, as reduções dos valores absolutos foram de 39, 2 e 44,4\% respectivamente.

Esses resultados mostram a dependência do posicionamento e da magnitude 


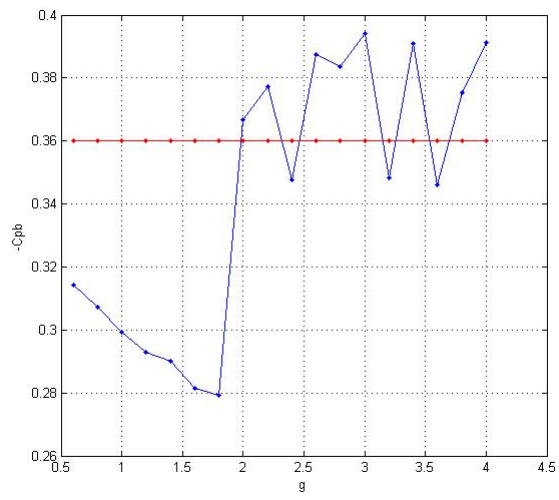

Figura 3.49: Coeficiente Médio de Pressão na Base do Cilindro $\overline{-C_{p b}}$ versus $g / d$, para $l / d=0,5$ e $R e=120$. Linha Vermelha, sem splitter plate; Linha azul, com splitter plate.

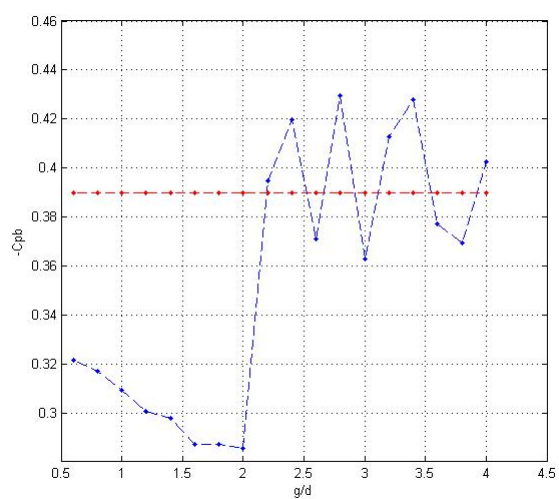

Figura 3.50: Coeficiente Médio de Pressão na Base do Cilindro $\overline{-C_{p b}}$ versus $g / d$, para $l / d=0,5$ e $R e=140$. Linha Vermelha, sem splitter plate; Linha azul, com splitter plate.

da redução do parâmetro em função de Re. Na faixa do adimensional que está sendo utilizada neste trabalho $(80 \leq R e \leq 140)$, as forças viscosas $\left(\frac{\mu}{D}\right.$ possuem relevância significativa,

Entretanto, como citado anteriormente, esta dependência não é vista em Igarashi (1982), pois seus experimentos estavam na ordem de $R e=10^{5}$, ou seja, as forças viscosas não possuem relevância frente as forças de inércia $(\rho \times V)$.

Para $l / d=1,0$, o comportamento das curvas não apresentou nenhum ponto destoante em relação as apresentadas por (IGARASHI, 1984).

Na simulação de $R e=80$, a redução foi de aproximadamente $29,0 \%$ em relação ao escoamento sem splitter plate. Já para $R e=100$, a redução foi de 32,3\%. Para $R e=120$ e 140, as reduções do módulo do coeficiente foram de 36,1 e $43,5 \%$ respectivamente. 


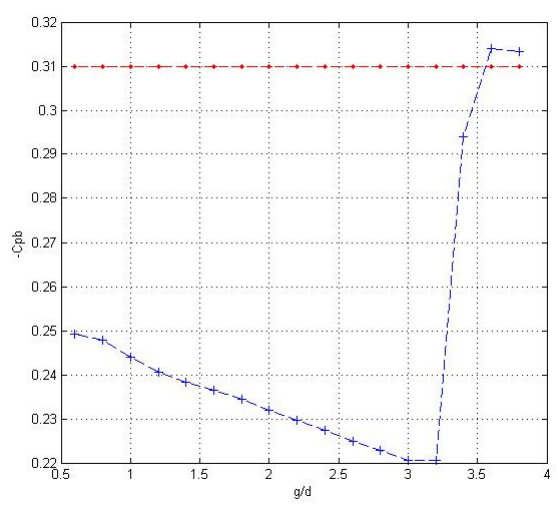

Figura 3.51: Coeficiente Médio de Pressão na Base do Cilindro $\overline{-C_{p b}}$ versus $g / d$, para $l / d=1,0$ e $R e=80$. Linha Vermelha, sem splitter plate; Linha azul, com splitter plate.

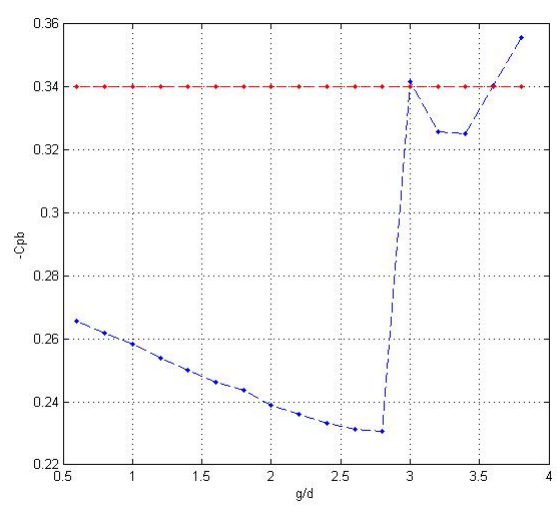

Figura 3.52: Coeficiente Médio de Pressão na Base do Cilindro $\overline{-C_{p b}}$ versus $g / d$, para $l / d=1,0$ e $R e=100$. Linha Vermelha, sem splitter plate; Linha azul, com splitter plate.

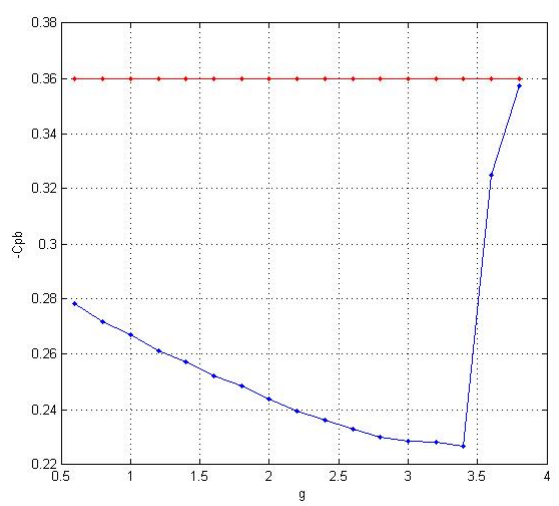

Figura 3.53: Coeficiente Médio de Pressão na Base do Cilindro $\overline{-C_{p b}}$ versus $g / d$, para $l / d=1,0$ e $R e=120$. Linha Vermelha, sem splitter plate; Linha azul, com splitter plate. 


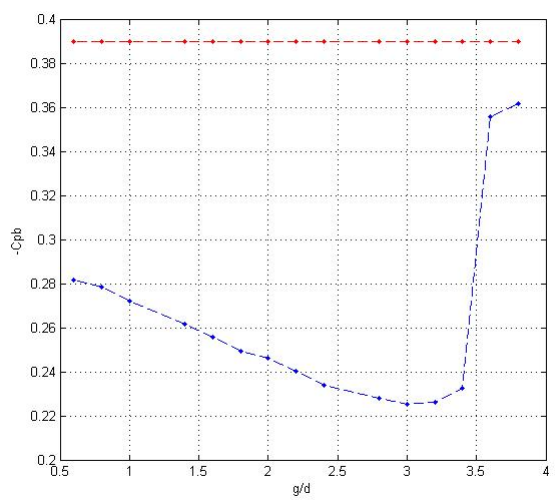

Figura 3.54: Coeficiente Médio de Pressão na Base do Cilindro $\overline{-C_{p b}}$ versus $g / d$, para $l / d=1,0$ e $R e=140$. Linha Vermelha, sem splitter plate; Linha azul, com splitter plate.

Neste trabalho foi apresentado apenas o coeficiente de pressão na base do cilindro, porém para trabalhos futuros, o estudo da distribuição de pressão ao longo do cilindro pode ser analisado e sua variação (ou até mesmo a busca de uma distribuição otimizada) em função da presença de splitter plates pode ser verificada.
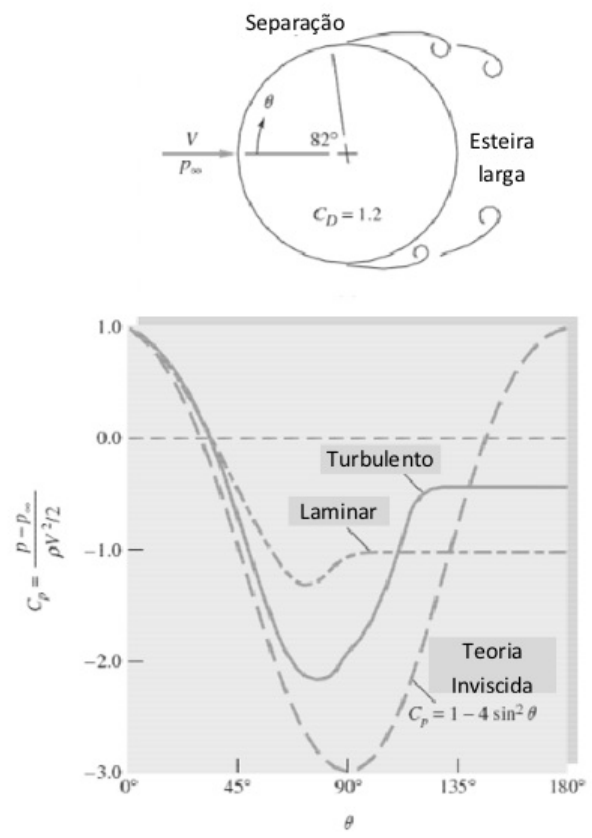

Figura 3.55: Distribuição de pressão ao longo do cilindro, adaptado de T.H. (2004)

Vale ressaltar que a faixa do número de Reynolds deste trabalho caracteriza um escoamento laminar, onde o mesmo é muito vulnerável ao gradiente adverso de pressão a jusante do cilindro. Em virtude disso, o ângulo de separação $(\theta)$ para esta faixa fica em torno de $82^{\circ}$, como apresentado na figura 3.55. 
De maneira resumida, a tabela 3.7 apresenta todos os resultados obtidos:

Tabela 3.7: Resumo dos resultados - Coeficiente de Pressão na Base do Cilindro

\begin{tabular}{cccc}
\hline Reynolds & l/d & g/d (mínimo) & Valor (Redução) \\
\hline 80 & 0,5 & 2,0 & $16,1 \%$ \\
80 & 1,0 & 3,2 & $29,0 \%$ \\
100 & 0,5 & 2,0 & $25,9 \%$ \\
100 & 1,0 & 2,4 & $32,3 \%$ \\
120 & 0,5 & 1,8 & $39,2 \%$ \\
120 & 1,0 & 3,4 & $36,1 \%$ \\
140 & 0,5 & 2,0 & $44,4 \%$ \\
140 & 1,0 & 3,0 & $43,5 \%$
\end{tabular}




\section{O MÉtodo AduUnto}

\subsection{Introdução}

O uso do CFD já é amplamente difundido em todos os ramos da mecânica dos fluídos e tem sido parte fundamental na análise da física do escoamento, complementando os ensaios experimentais que também podem ser realizados.

Recentemente, com a pesquisa e desenvolvimento de métodos inversos de otimização, a necessidade de se realizar cálculos para o estudo de diversas configurações em pouco tempo tornou-se evidente, o que abriu as portas para o aprimoramento do CFD. No momento em que o custo (principalmente no tempo de cálculo) para se realizar uma simulação computacional ficou menor que o preparo de todo um aparato experimental, inúmeras possibilidades surgiram dentro de projetos de otimização pois tornou-se viável a integração entre o CFD e um algoritmo de otimização buscando o extremo de um dado parâmetro de projeto.

Nesta linha de desenvolvimento, o método adjunto surgiu como uma potencial ferramenta de integração entre uma otimização e o CFD por inúmeras razões que serão discutidas neste capítulo. A principal delas é a grande flexibilidade de escolha da função objetivo e também das equações que irão governar o escoamento, podendo assim utilizar o mesmo algoritmo para inúmeras aplicações de engenharia.

\subsection{Revisão Bibliográfica}

Este método foi proposto inicialmente por Pirroneau (1973) em problemas elípticos, sendo extendido para escoamento transônicos por Jameson (1988a). Ao longo dos anos, o método adjunto foi se tornando um assunto de grande interesse e pesquisa, com Jameson sendo um dos principais pesquisadores, publicando inúmeros artigos que geraram a difusão de seu trabalho, trazendo novos pesquisadores para desenvolverem o tema onde pode-se citar Jameson (1988b), Cabuk, Sung e Modi (1991), Taasan, Kuruvila e Salas (1992), Kuruvila, Taasan e Salas 
(1994), Jameson (1995), Santos (1995), Jameson, Martinelli e Pierce (1997), Kim (2001), Mohammadi e Pirroneau (2001), Alonso e Kroo (2002). Inúmeras aplicações surgiram, desde aplicações em reatores nucleares a estudos hidrodinâmicos e aerodinâmicos (CACUCI et al., 1980; HALL; CACUCI, 1983).

Em aerodinâmica, o desenvolvimento do método adjunto foi aplicado em escoamentos internos e externos em regime permamente (NADARAJAH, 2003; KIM; NAKAHASHI, 2005; KIM; ALONSO; JAMESON, 2004; KIM et al., 2001; QIAO; QIN; YANG, 2002; DUTA; GILES; CAMPOBASSO, 2002) e mais recentemente, em escoamentos em regime transitório (THOMAS; HALL; DOWELL, 2005; NADARAJAH; JAMESON, 2006; NADARAJAH; JAMESON, 2007; MANI; MAVRIPLIS, 2008). Uma área diferente de pesquisa foi aberta no estudo de análises de erro (GILES; PIERCE; SüLI, 2004; GILES; SüLI, 2002) e no estudo de adaptação de malha (VENDITTI; DARMOFAL, 1999; VENDITTI; DARMOFAL, 2000; GILES; SüLI, 2002; VENDITTI; DARMOFAL, 2003). Esses assuntos utilizam as variáveis adjuntas para aumentar a precisão dos funcionais que medem os requisitos desejados para a solução do escoamento (GILES; PIERCE, 1998b; GILES; PIERCE, 1999a; GILES; PIERCE, 1999b; PIERCE; GILES, 2004).

As principais aplicações de interesse da aerodinâmica utilizam funções objetivos que normalmente dependem das variáveis do escoamento, da geometria do corpo imerso e também das condições de contorno (JAMESON; MARTINELLI; PIERCE, 1998; JAMESON; SRIRAM; MARTINELLI, 2003). Estas variáveis, por sua vez, são dependentes de um conjunto de parâmetros finitos de projeto que formulam o problema para resolução. Sob essas circunstâncias, uma maneira trivial de estimativa do gradiente de sensibilidade da função objetivo é a pertubação de cada parâmetro individualmente para avaliar a sua sensibilidade e então o cálculo do gradiente de fato pelo método de diferenças finitas, algo parecido com o que foi feito no capítulo anterior.

Este procedimento claramente requer uma solução convergida do escoamento para cada pertubação das variáveis de projeto. Consequentemente, se o número de variáveis de projeto for alto, mesmo com todo desenvolvimento do CFD, o custo computacional para esse procedimento torna este método proibitivo.

Alternativamente a isto, o método adjunto, em sua essência, impõe na sua formulação que as equações que governam o escoamento sejam restrições no domínio das variáveis de projeto, ou seja, apenas configurações realizáveis serão obtidas. Assim, não haverá a necessidade de simulações adicionais do escoamento. Como resultado, o método oferece um custo extremamente atrativo para avaliação do 
gradiente de sensibilidade, tornando ainda mais vantajoso se o número de variáveis aumenta.

Uma introdução mais geral sobre o método adjunto é apresentado em (CACUCI et al., 1980) e este trabalho irá descrever seus principais tópicos. Inicia-se a formulação apresentando o funcional de otimização, conhecido como medida de mérito, em sua forma genérica:

$$
R[\mathbf{X}, \alpha]=\int_{\rho} F[\mathbf{X}(\rho), \alpha(\rho), \rho] \mathrm{d} \rho
$$

Onde o vetor $\mathbf{X}$ representa as variáveis do escoamento, o vetor $\rho$ representa as coordenadas do espaço físico e finalmente o vetor $\alpha$ representa as variáveis de projeto que controlam o sistema. Na forma genérica, tem-se que:

$$
\begin{aligned}
\mathbf{X}(\rho) & =\left[X_{1}(\rho), \ldots X_{K}(\rho)\right] \\
\rho & =\left(\rho_{1}, \ldots, \rho_{J}\right) \\
\alpha(\rho) & =\left[\alpha_{1}(\rho), \ldots, \alpha_{I}(\rho)\right]
\end{aligned}
$$

A primeira variação de Gâteaux (LUSTERNICK; SOBOLEV, 1961) da equação 4.1 produz a expressão:

$$
\delta R=\underbrace{\int F_{X}^{\prime} \delta \mathbf{X}(\rho) \mathrm{d} \rho}_{\delta R_{X}}+\underbrace{\int F_{\alpha}^{\prime} \delta \alpha(\rho) \mathrm{d} \rho}_{\delta R_{\alpha}}
$$

Onde o primeiro termo $\delta R_{X}$ representa a parte física da variação, o segundo termo $\delta R_{\alpha}$ representa a parte geométrica das aplicações de interesse. Em geral, o termo $F_{\alpha}^{\prime} \delta \alpha(\rho)$ é conhecido e sua variação $\delta R_{\alpha}$ pode ser obtida analiticamente. Entretanto, há uma grande dificuldade na estimativa do primeiro termo de $\delta R$, ou seja, $\delta R_{X}$. Como a variação $\delta \mathbf{X}(\rho)$ é raramente conhecida em uma formulação analítica, consequentemente $F_{X}^{\prime}$ não será obtido facilmente. Assim, para se obter essa variação, novamente há a necessidade do método de diferenças finitas, o que não é desejável.

Para contornar este problema, será utilizado a essência do método adjunto mencionada anteriormente que é a imposição das equações que governam a física do escoamento como restrições na medida de mérito. Por fim, assume-se que o sistema será governando por um sistema $\mathbf{N}$ de $K$ equações diferenciais não 
lineares e o operador $\mathbf{B}$ representa as condições do contorno do sistema. Chegase portanto a seguinte formulação:

$$
\begin{aligned}
\mathbf{N}[\mathbf{X}(\rho), \alpha] & =\mathbf{Q}(\rho, \alpha) \\
\mathbf{B}[\mathbf{X}(\rho), \alpha]_{s} & =0
\end{aligned}
$$

Onde o índice [ ] implica que as condições serão impostas nas devidas fronteiras. É também assumido que o produto interno pode ser definido no espaço na forma.

$$
\langle\Phi, \Psi\rangle=\int_{\rho} \Phi \cdot \Psi \mathrm{d} \rho
$$

Essa restrição do problema adicionada a medida de mérito forma a função objetivo aumentada, que será o problema variacional com restrições:

$$
G(\mathbf{X}, \alpha, \Phi, \beta, a)=R[\mathbf{X}, \alpha]-\langle\Phi, \mathbf{N}-\mathbf{Q}\rangle-\langle\beta, \mathbf{B}\rangle_{s}-\left\langle\mathbf{a}, \alpha-\alpha_{o}\right\rangle
$$

Como as restrições são normalmente não holonômicas, elas foram introduzidas como multiplicadores de Lagrange $\Phi, \beta$ e a nos três últimos funcionais da equação 4.9. O primeiro deles impõe as equações que governam o escoamento, enquanto a segunda impõe as condições de contorno correspondentes. A terceira restrição certifica que as variáveis que controlam o sistema assumam precisamente os valores $\alpha=\alpha_{o}$ que correspondem a configuração base.

Para determinar o extremo de $G$, é necessário calcular a sua variação, $\delta G$. Com este propósito, a abordagem será iniciada considerando as equações 4.6 e 4.7. As derivadas de Fréchet são dadas como:

$$
\begin{aligned}
\mathbf{L} \delta \mathbf{X} & =\mathbf{S} \delta \alpha \\
\mathbf{B}_{X}^{\prime} \delta \mathbf{X} & =-\mathbf{B}_{\alpha}^{\prime} \delta \alpha
\end{aligned}
$$

Onde os operadores $\mathbf{L}$ e $\mathbf{S}$ são definidos como $\mathbf{L} \equiv \mathbf{N}_{X}^{\prime}$ e $\mathbf{S} \equiv \mathbf{Q}_{\alpha}^{\prime}-\mathbf{N}_{\alpha}^{\prime}$. O primeiro do termos representa a forma linearizada das equações que governam o escoamento, enquanto o segundo reune as variações dos parâmetros de controle. 
O primeiro termo da equação 4.9 é apenas a variação de $R$, cuja a primeira derivada é dada pela equação 4.5. Por outro lado, é possível transformar os termos em produtos internos como na equação 4.8 .

Como no segundo termo da equação 4.9, deve-se calcular a primeira derivada de Gâteaux e substituir pela equação 4.10. Então, utilizando o teorema de Gauss, que pode transferir os operadores diferenciais do vetor de estado $\mathbf{X}$ em multiplicadores de Lagrange $\Phi$. Isto leva ao seguinte resultado:

$$
\langle\Phi, \mathbf{L} \delta \mathbf{X}\rangle=\left\langle\mathbf{L}^{*} \Phi, \delta \mathbf{X}\right\rangle-P[\Phi, \delta \mathbf{X}]_{s}
$$

Onde o termo $P[\Phi, \delta \mathbf{X}]_{s}$ é um concomitante bilinear (CACUCI et al., 1980; MORSE; FESHBACH, 1953a). Novamente, o índice [ $]_{s}$ tem o mesmo significado citado anteriormente. Além disso, o primeiro termo da equação 4.12 contém L*, que é o operador adjunto de $\mathbf{L}$

Finalmente, através do cálculo da derivada de Gâteax dos funcionais remanescentes e combinando eles com os resultados acima, obtém-se a primeira derivada da função objetivo aumentada, $\delta G$ :

$$
\begin{aligned}
\delta G= & -\langle\delta \Phi, \mathbf{N}-\mathbf{Q}\rangle-\langle\delta \beta, \mathbf{B}\rangle_{s}-\left\langle\delta a, \alpha-\alpha_{o}\right\rangle+\left\langle\mathbf{L}^{*} \Phi-F_{X}^{\prime}, \delta \mathbf{X}\right\rangle+ \\
& -\left\langle\beta, \mathbf{B}_{X}^{\prime} \delta X\right\rangle_{s}-\left[\left\langle P_{1}(\Phi), \mathbf{B}_{X}^{\prime} \delta \mathbf{X}\right\rangle_{s}+\left\langle\mathbf{B}^{*}(\Phi), \mathbf{A} \delta \mathbf{X}\right\rangle_{s}\right]+\left\langle\mathbf{F}_{\alpha}^{\prime}, \delta \alpha\right\rangle+ \\
& +\langle\Phi, \mathbf{S} \delta \alpha\rangle-\langle\mathbf{a}, \delta \alpha\rangle-\left\langle\beta, \mathbf{B}_{\alpha}^{\prime} \delta \alpha\right\rangle_{s}
\end{aligned}
$$

Onde $\delta R$ já foi substituído pela equação 4.5 .

Adicionalmente, o concomitante bilinear $P[\Phi, \delta \mathbf{X}]_{s}$ da equação 4.12 foi decomposto em dois termos entre colchetes. Ambos são produtos escalares e somente serão calculados nas fronteiras apropriadas. O primeiro destes termos envolve $P_{1}(\Phi)$ e o operador de fronteira linearizado $\mathbf{B}_{X}^{\prime} \delta \mathbf{X}$, enquanto o segundo termo envolve o termo $\mathbf{B}^{*}(\Phi)$, que representa o operador adjunto de fronteira linearizado e o termo $\mathbf{A} \delta \mathbf{X}$. A decomposição de $P$ não é única, como também não é para $P_{1}$ e $\mathbf{A}$, a única restrição que normalmente é imposta no procedimento é que o operador A seja linearmente independente de $\mathbf{B}_{X}^{\prime}$.

Como resultado disto, a determinação do problema de contorno do método adjunto leva a uma decomposição não única que, em princípio, permite que se faça das equações adjuntas, equações bem postas. Após isso, deverá existir alguma manipulação matemática no lado esquerdo da equação para certificar a 
consistência do problema. O extremo da medida de mérito corresponde a:

$$
\delta G=0 \forall\{\delta \mathbf{X}, \delta \alpha, \delta \Phi, \delta \beta, \delta \mathbf{a}\} \in\{\text { Locus de realizabilidade }\}
$$

Onde a equação 4.14 impõe os seguintes requisitos:

I.- As equações que governam a física do escoamento (Eq. 4.6) e suas respectivas condições de contorno (Eq. 4.7) deverão ser satisfeitas pelas configurações base. Adicionalmente, os parâmetros de controle deverão assumir valores estabelecidos, $\alpha=\alpha_{o}$. Estes requisitos implicam que o primeiro dos três termos da equação 4.13 serão nulos.

II.- Impondo a condição:

$$
\beta=-P_{1}(\Phi)
$$

Ela levará o quinto e sexto termos da equação 4.13 a zero. Esta equação em particular soluciona $\beta$ em termos de $\Phi$.

III.- O vetor $\Phi$ deverá satisfazer a equação adjunta,dada por:

$$
\mathbf{L}^{*} \Phi-F_{X}^{\prime}=0
$$

que aparece no quarto termo da equação 4.13. A correspondente condição de contorno será dada pelo operador:

$$
\mathbf{B}^{*}(\Phi)=0
$$

que se origina do sétimo termo da equação 4.17. Esta equação deverá determinar $\Phi$ nas fronteiras, juntamente $\operatorname{com} \beta$.

IV.- O vetor a é especificado com a seguinte condição:

$$
\langle\mathbf{a}, \delta \alpha\rangle=\left\langle\mathbf{F}_{\alpha}^{\prime}, \delta \alpha\right\rangle+\langle\Phi, \mathbf{S} \delta \alpha\rangle-\left\langle\beta, \mathbf{B}_{\alpha}^{\prime} \delta \alpha\right\rangle_{s}
$$

Onde estão contidos todos os termos remanescentes quando $\delta G=0$. De fato, esta é a parte correspodente do gradiente de sensibilidade $\delta R$, que será apresentada a seguir

Para provar as afirmações acima sobre o gradiente de sensibilidade, basta reconhecer que as equações 4.6 e 4.7 que governam o escoamento são satisfeitas para uma dava variação $\Delta G$ de qualquer magnitude (CACUCI et al., 1980). Então, a partir da definição de $G$ na equação 4.9 , isto leva a: 


$$
\begin{aligned}
& \Delta G=\Delta R-\langle\mathbf{a}, \Delta \alpha\rangle \\
& \text { for }\left\{\begin{array}{l}
\Delta G \equiv G\left(X_{2}, \alpha_{2} ; \Phi_{2}, \beta_{2}, a_{2}\right)-G\left(X_{1}, \alpha_{1} ; \Phi_{1}, \beta_{1}, a_{1}\right) \\
\Delta R \equiv R\left(X_{2}, \alpha_{2}\right)-R\left(X_{1}, \alpha_{1}\right) \\
\Delta \alpha \equiv \alpha_{2}-\alpha_{1}
\end{array}\right.
\end{aligned}
$$

Em particular, para uma variação infinitesimal $\Delta G \rightarrow \delta G$, sob as condições acima e com $\Phi, \alpha$ e $\beta$ atendendo totalmente os requisitos de I a IV, isto corresponde ao valor estacionário de $G$. Assim, pode-se escrever que:

$$
\begin{aligned}
\delta G & =\delta R-\langle\mathbf{a}, \delta \alpha\rangle=0 \\
\delta R & =\langle\mathbf{a}, \delta \alpha\rangle \\
\delta R & =\left\langle\mathbf{F}_{\alpha}^{\prime}, \delta \alpha\right\rangle+\left\langle\Phi,\left(\mathbf{Q}_{\alpha}^{\prime}-\mathbf{N}_{\alpha}^{\prime}\right) \delta \alpha\right\rangle+\left\langle P_{1}(\Phi), \mathbf{B}_{\alpha}^{\prime} \delta \alpha\right\rangle_{s}
\end{aligned}
$$

Onde as equações $4.15,4.18$ e a definição de $\mathbf{S}$ são usadas. Com a equação 4.20 acima, pode-se estimar o grandiente de sensibilidade com base na solução adjunta $\Phi$ e com as variações dos parâmetros de controle, $\delta \alpha$.

É interessante notar que todas as variações físicas $\delta \mathbf{X}$ são removidas da expressão do gradiente. Além disso, o primeiro termo da equação 4.20 é precisamente $\delta R_{\alpha}$, considerando que o segundo termo avalia o efeito direto de $\delta \alpha$ nas equações que governam o escoamento, o terceiro faz o mesmo com as condições de contorno. Na literatura, o problema do escoamento e o problema adjunto são normalmente nomeados como problema primal e dual, respectivamente.

Apesar da simples fundamentação conceitual, há alguns aspectos no problema adjunto, que devem ser abordados com cuidado:

- A derivação do operador adjunto $\mathbf{L}^{*}$ por métodos analíticos pode ter gerar grandes dificuldades embora há informações a respeito disso na literatura.

- Como mencionado acima, a decomposição do concomitante bilinear não é única. De fato, é uma questão importante pois irá determinar de o problema adjunto estará bem condicionado.

- Deve-se considerar que as variáveis adjuntas são entidades puramente matemáticas. Diferente dos problemas de mecânica do fluídos, nem sempre é possível se beneficiar do conhecimento de Física para formular o problema adjunto. 
- A revisão bibliográfica parece indicar que o estudo das condições de contorno adjuntas ainda não receberam a atenção devida.

Sob essas circunstâncias, o método adjunto desenvolveu-se duas formulações distintas: Discreta e Contínua. Na primeira formulação, o operador adjunto é obtido com base nas equações que governam o escoamento em sua forma discreta. Já a segunda formulação, as equações adjuntas são derivadas analiticamente a partir das equações que governam o escoamento (GILES; PIERCE, 1997; GILES; PIERCE, 1998a; GILES; PIERCE, 2000; GILES; PIERCE, 2001). Comparações entre as duas formulações são descritas na literatura (JAMESON; SRIRAM; MARTINELLI, 2003; NADARAJAH; JAMESON, 2000; KIM; JAMESON, 1999), e não são indicadas diferenças significativas entre elas em termos de eficiência no processo de otimização.

O desenvolvimento acima pode passar ao leitor uma visão geral das aplicações e novos desenvolvimentos do método adjunto em otimizações aerodinâmicas, além de conhecer a sua origem a partir dos projetos inversos. Porém, um artigo recente elaborado por Barkley et al. (BARKLEY; BLACKBURN; SHERWIN, 2002) elabora uma nova abordagem de pesquisa. Na referência, os autores usam o método adjunto nas equações de Navier-Stokes para o escoamento incompressível, para investigar o escoamento em regime transiente e suas instabilidades hidrodinâmicas. A idéia atraiu a atenção e originou este trabalho, utilizando a mesma formulação presente na referência para a aplicação do método adjunto em problemas de interesse da indústria do Petróleo.

Entretanto há alguns aspectos da formulação que devem ser mudados: Primeiro, as equações adjuntas e do escoamento devem ser transformadas em sua forma generalizada, de forma a acomodar as variações geométricas que são intrísecas nestas aplicações. Adicionalmente, o problema das condições de contorno adjuntas é fundamentalmente diferente dos problemas de instabilidades dinâmicas.

De maneira a explorar estes tópicos, o capítulo 4.3 será totalmente dedicado a discussão e aprofundamento do problema adjunto, bem como a derivação de suas equações e condições de contorno que se aplicam ao estudo de caso em questão. Em particular para o problema de contorno, os concomitantes bilineares serão decompostos de uma maneira nova, para garantir que o problema esteja bem condicionado. 


\subsection{Modelagem da Solução Adjunta baseada nas equações de Navier Stokes}

Esta seção foi dedicada ao desenvolvimento do método adjunto aplicado aos escoamentos viscosos, utilizando como base o trabalho produzido em (VOLPE et al., 2012). Será discutida a derivação das equações adjuntas formuladas a partir das equações de Navier-Stokes, com suas condições de contorno e variações no tempo.

A aproximação apresentada nesta derivação é análoga ao que foi proposto por Jameson e seus colaboradores (NADARAJAH; JAMESON, 2007; JAMESON; MARTINELLI; PIERCE, 1998). Entretanto, há uma diferença entre o que foi proposto por Cacuci em relação as condições de contorno não serem explicitamente impostas no problema variacional (CACUCI et al., 1980). A comparação entre as duas formulações não será escopo deste trabalho e está presente em (VOLPE et al., 2012).

Por outro lado, as equações deduzidas também são similares ao trabalho apresentado por Barkley et al. mas com importantes diferenças relacionadas as condições de contorno (BARKLEY; BLACKBURN; SHERWIN, 2002). A formulação apresentada na sequência possui similaridades com as hipóteses discutidas em (VOLPE; SANTOS, 2009; HAYASHI, 2009).

\subsubsection{Introdução}

Para os problemas de otimização que serão considerados neste trabalho, o objetivo principal será encontrar uma situação de escoamento que represente os extremos de uma certa medida de mérito. Esta medida é normalmente uma função das variáveis do escoamento, como as apresentadas na equação 4.1. Esta medida poderia ser uma integral sobre todo domínio do escoamento, ou uma integral de superfície sobre uma certa fronteira, como a superfície de um corpo.

Este último caso possui um interesse especial para este trabalho pois essas integrais de fronteira resultam os coeficientes de força de arrasto $\left(C_{d}\right)$ e sustentação $\left(C_{l}\right)$ além de ser possível realizar otimizações através de projeto inverso com as distribuições de pressão calculadas.

Um exemplo pode ser considerado como uma força que um fluído exerce sobre 
um corpo, quando é projetada em uma certa direção:

$$
I_{o}=\frac{1}{T} \int_{0}^{T} \oint_{B} G(\mathbf{n} \cdot \boldsymbol{\sigma} \cdot \mathbf{e}) \mathrm{d} S \mathrm{~d} t
$$

Onde $\boldsymbol{\sigma}$ é o tensor das tensões do escoamento, $\boldsymbol{\sigma}=\boldsymbol{\tau}-p \mathbf{I}$, e os vetores $\mathbf{n}$ e e representam a direção normal em relação a superficie do corpo e a direção da projeção respectivamente. Adicionalmente, $G$ é assumido como sendo uma função genérica destes argumentos.

Como os escoamentos são isotérmicos incompressíveis, a física do problema é totalmente descrita pelos campos de velocidade e pressão $(\mathbf{u}, p)$. Estes campos descrevem o domínio do sistema e definem o vetor de estado como sendo:

$$
\mathbf{q} \equiv\left(u^{1}, u^{2}, u^{3}, p\right)^{T} \Rightarrow\left\{\begin{array}{l}
q^{i}=u^{i} \text { for } i=1,2,3 \\
q^{4}=p
\end{array}\right.
$$

O espaço físico é representado por $\left(x^{i^{\prime}}, t\right)$ em coordenadas cartesianas. O domínio do escoamento $\mathcal{D}$ e passo no tempo também definem o domínio do problema $\Omega=\mathcal{D} \times(0, T)$.

O objetivo do problema é a busca por uma geometria do corpo que proporcione o mínimo da função objetivo (4.21), dada as condições do escoamento externo. Neste caso, a geometria do corpo é considerada como totalmente descrita por um número finito de parâmetros de projeto.

$$
S\left(x^{i^{\prime}} ; a^{k}\right) \Leftrightarrow S\left(\xi^{j} ; a^{k}\right)
$$

Por uma conveniência da formulação matématica, é importante ressaltar que a mesma geometria pode ser representada pelas coordenadas cartesianas $\left(x^{i^{\prime}}\right)$ ou pelas coordenadas generalizadas $\left(\xi^{j}\right)$. Para termos distinção entre as duas representações, a primeira será representada por $S^{\prime}$ e a segunda por $S$.

A idéia do problema consiste em minimizar $I_{o}$ a partir das variações dos parâmetros de projeto. Portanto, os $a^{k}$ podem ser vistos como as variáveis de controle do sistema físico e o valor $I_{o}$ pode ser considerado como a medida de mérito. Para o alcance do extremo desta medida de mérito, uma informação necessária é a sensibilidade desta relação com as variáveis de projeto. Em primeira ordem, este valor pode ser estimado pelo gradiente de sensibilidade: $\partial I_{o} / \partial a^{k}$. 
Entretanto, deve ser levado em conta que tanto a integral de superfície do corpo e as variações físicas do escoamento podem mudar o resultado da equação 4.21, sempre que ocorre alguma variação no valor de $a^{k}$. Logo, é conveniente separar as variações físicas do escoamento das variações geométricas, usando a tranformação espacial $\left(x^{i^{\prime}} \rightleftharpoons \xi^{j}\right)$, no qual a superfície do corpo é mapeada em um outro sistema de coordenadas.

Os detalhes da dedução algébrica estão apresentados sob forma de apêndice no final deste trabalho (Apêndice A), porém o raciocínio deste procedimento é simples: A representação geométrica da superfície do corpo no espaço transformado é fixa. Ela não será alterada com as variações $\delta a^{k}$ e também pela consequente alteração da geometria física. As únicas variáveis que se alteram com $\delta a^{k}$ são os operadores de transformação $\beta$ e $\beta^{-1}$, bem como os termos da métrica do espaço transformado.

A partir disso, as variações físicas e geométricas podem ser separadas, simplificando o problema variacional consideravelmente com o domínio de integração presente no espaço transformado (GELFAnd; FOMIN, 1963). Como exemplo, o vetor velocidade abaixo é transformado da seguinte forma:

$$
\begin{aligned}
u^{i} & =\beta_{j^{\prime}}^{i} u^{j^{\prime}} \\
\delta u^{i} & =\underbrace{\beta_{j^{\prime}}^{i} \delta u^{j^{\prime}}}_{\left(\delta u^{i}\right)_{P}}+\underbrace{\delta \beta_{j^{\prime}}^{i} u^{j^{\prime}}}_{\left(\delta u^{i}\right)_{M}} \\
(\delta)_{T} & ={ }^{i}
\end{aligned}
$$

A variação total é a soma dos dois termos, sendo o primeiro termo $\left(\delta u^{i}\right)_{P}$ representando a parte física da variação no espaço transformado, enquanto o segundo termo $\left(\delta u^{i}\right)_{M}$ corresponde a variação geométrica no espaço físico, com as condições do escoamento fixas.

Aplicando agora a transformação na equação 4.21 e calculando seu gradiente de sensibilidade em função das variáveis de projeto, obtém-se a seguinte expressão:

$$
\begin{aligned}
I_{o} & =\frac{1}{T} \int_{0}^{T} \oint_{B} G\left|\frac{d S^{\prime}}{d S}\right| \mathrm{d} S \mathrm{~d} t \\
\frac{\partial I_{o}}{\partial a^{k}} & =\frac{1}{T} \int_{0}^{T} \oint_{B}\left[\frac{\partial G}{\partial q^{i}} \frac{\partial q^{i}}{\partial a^{k}}\left|\frac{d S^{\prime}}{d S}\right|+G \frac{\partial}{\partial a^{k}}\left|\frac{d S^{\prime}}{d S}\right|\right] \mathrm{d} S \mathrm{~d} t
\end{aligned}
$$


Onde a razão entre os elementos de área $\left|\mathrm{d} S^{\prime} / \mathrm{d} S\right|$ foi derivada a partir das relações de área entre os espaços físico e transformado. O primeiro termo da integral que compõe o gradiente de sensibilidade consiste na variação das propriedades físicas do escoamento em relação as variáveis de projeto, enquanto o segundo termo é a medição de como a geometria do corpo se altera em relação a variação $\delta a^{k}$. Assim, fica claro que foi possível a separação dos termos físicos e geométricos do problema. Adicionalmente, os termos $\left(\partial G / \partial q^{i}\right)$ e $\left(\partial\left|d S^{\prime} / d S\right| / \partial a^{k}\right)$ são conhecidos a partir das expressões de $G$ e $S^{\prime}$.

Como discutido no capítulo 4, o problema principal da equação 4.24 é o fato que o termo que depende das variações do escoamento $\left(\partial q^{i} / \partial a^{k}\right)$ que raramente conhecido na forma analítica. Para obtê-lo, é necessário uma série de simulações do escoamento, sendo cada uma com uma variação individual de cada parâmetro de projeto $\delta a^{k}$, mantendo os outros constantes. Desta situação, partiu-se para a idéia de restringir as variações do escoamento dentro de um universo realizável.

\subsubsection{Equações Adjuntas}

Para considerar apenas o universo de soluções realizáveis, as restrições serão as próprias equações de Navier-Stokes, que foram escritas na forma de escoamentos incompressíveis e isotérmicos:

$$
\left\{\begin{aligned}
\partial_{o} \mathbf{u}+(\mathbf{u} \cdot \nabla) \mathbf{u}+v \nabla p-\nu \nabla^{2} \mathbf{u} & =0 \\
\nabla \cdot \mathbf{u} & =0
\end{aligned}\right.
$$

Onde $\mathbf{u}$ é a velocidade do fluído; $p$, a pressão; $v$, é o volume específico do fluído e $\nu$, a viscosidade cinemática, assumida como constante.

As equações são impostas no problema variacional como restrições não-holonômicas (GELFAND; FOMIN, 1963), que leva a obtenção da função objetivo aumentada:

$$
\begin{aligned}
I & =\frac{1}{T} \int_{0}^{T} \oint_{B} G(\mathbf{n} \cdot \boldsymbol{\sigma} \cdot \mathbf{l})\left|\frac{d S^{\prime}}{d S}\right| \mathrm{d} S \mathrm{~d} t-\frac{1}{T}\left\{\int_{\Omega} \theta \nabla \cdot \mathbf{u} \mathrm{d} \Omega+\right. \\
& \left.+\int_{\Omega} \boldsymbol{\psi} \cdot\left[\partial_{o} \mathbf{u}+(\mathbf{u} \cdot \nabla) \mathbf{u}+v \nabla p-\nu \nabla^{2} \mathbf{u}\right] \mathrm{d} \Omega\right\}
\end{aligned}
$$

Onde $\theta$ e $\psi$ representam os multiplicadores de Lagrange do problema variaci- 
onal. Como mencionado acima, o trabalho de Jameson não impõe as condições de contorno do escoamento como restrições do problema variacional, como Cacuci. Neste caso, a integração sobre o o domínio do escoamento como um todo $(\Omega)$ é representada pela notação:

$$
\int_{\Omega} f\left(x^{j^{\prime}}, t\right) \mathrm{d} \Omega \equiv \int_{0}^{T} \int_{\mathcal{D}} f\left(x^{j^{\prime}}, t\right) \mathrm{d} V \mathrm{~d} t=\int_{0}^{T} \int_{\mathcal{D}} f\left(\xi^{k}, t\right) J \mathrm{~d} \xi^{1} \mathrm{~d} \xi^{2} \mathrm{~d} \xi^{3} \mathrm{~d} t
$$

O símbolo $J$ representa o Jacobiano da transformação, que leva o sistema cartesiano do espaço físico para o espaço de coordenadas generalizadas no espaço transformado.

Como visto na equação 4.24, a variação da função de mérito aumentada (equação 4.26) é calculada em função das variações físicas e geométricas do sistema. Portanto, isto implica que as equações de Navier Stokes também deverão ser resolvidas desta forma. No espaço de estado, estas equações podem ser escritas na forma do operador $\mathcal{N} \mathbf{q}=0$, onde $\mathcal{N}$ definido em (BARKLEY; BLACKBURN; SHERWIN, 2002):

$$
\left(\begin{array}{c|c}
\partial_{o}+(\mathbf{u} \cdot \nabla)-\nu \nabla^{2} & v \nabla \\
\hline \nabla \cdot & 0
\end{array}\right)\left(\begin{array}{c}
\mathbf{u} \\
\hline p
\end{array}\right)=\left(\begin{array}{c}
0 \\
\hline 0
\end{array}\right)
$$

A partir das condições de escoamento consideradas, é razoável assumir que as variáveis $\mathbf{q}=(\mathbf{u}, p)^{T}$ contínuas e diferenciáveis dentro do domínio do escoamento. Como resultado, é possível utilizar a derivada de Fréchet para se calcular a primeira variação das equações (CACUCI et al., 1980; LUSTERNICK; SOBOLEV, 1961).

$$
\mathcal{N}_{, q} \delta \mathbf{q}=\frac{\partial}{\partial \varepsilon}[\mathcal{N}(\mathbf{q}+\varepsilon \delta \mathbf{q})]_{\varepsilon=0}=0
$$

Com esta formulação, torna-se fácil calcular a primeira derivada em relação as variáveis de estado $\delta \mathbf{q}$. O resultado está apresentado abaixo:

$$
\left\{\begin{aligned}
\partial_{o} \delta u^{i}+\left.\left[u^{i} \delta u^{j}+u^{j} \delta u^{i}+g^{i j} v \delta p-\nu\left(\left.\delta u^{i}\right|^{j}+\left.\delta u^{j}\right|^{i}\right)\right]\right|_{j} & =0 \\
\left.\delta u^{i}\right|_{i} & =0
\end{aligned}\right.
$$

Onde o símbolo ()$_{P}$ foi retirado por simplicidade, no entendimento de que todas as variáveis métricas foram mantidas constantes para se obter a equação 4.30. É 
possível notar que a forma completa do tensor das tensões foi mantida na variação física da equação de momento,

$$
v \delta \tau^{i j}=\nu\left(\left.\delta u^{i}\right|^{j}+\left.\delta u^{j}\right|^{i}\right)
$$

Sendo que as razões desta formulação ficarão mais claras adiante.

No apêndice A, está mostrando que as Equações de Navier-Stokes nas formas extendida e reduzida são equivalentes em termos físicos, porém a forma reduzida é muito mais simples que a forma extendida em relação aos termos métricos e por isso, será utilizada na estimativa do gradiente de sensibilidade.

$$
\left\{\begin{array}{r}
\partial_{o}\left(\delta J u^{r^{\prime}}\right)+\left[\delta\left(J \beta_{n^{\prime}}^{j}\right)\left(u^{r^{\prime}} u^{n^{\prime}}+g^{r^{\prime} n^{\prime}} p v-\nu u_{, p^{\prime}}^{r^{\prime}} g^{p^{\prime} n^{\prime}}\right)\right]_{, j}=0 \\
{\left[\delta\left(J \beta_{q^{\prime}}^{k}\right) u^{q^{\prime}}\right]_{, k}=0}
\end{array}\right.
$$

Onde todas as quantificações físicas são mantidas constantes na derivação e o símbolo ( $)_{M}$ foi retirado por conveniência analogamente a equação 4.30

Assim, a primeira variação da função objetivo aumentada (Eq. 4.26) é obtida com a combinação das equações 4.30 e 4.32 .

$$
\begin{aligned}
& T \delta I=\underbrace{\int_{0} \oint_{B}\left(\frac{\partial G}{\partial p} \delta p+\frac{\partial G}{\partial \mathcal{F}^{k}} \delta \mathcal{F}^{k}\right)\left|\frac{d S^{\prime}}{d S}\right| \mathrm{d} S \mathrm{~d} t}_{I_{1}}+\underbrace{\left.\int_{\Omega} \theta \delta u^{k}\right|_{k} \mathrm{~d} \Omega}_{I_{2}}+ \\
& -\underbrace{\int_{\Omega} \psi_{i}\left\{\partial_{o} \delta u^{i}+\left.\left[u^{i} \delta u^{j}+u^{j} \delta u^{i}+g^{i j} v \delta p-\nu\left(\left.\delta u^{i}\right|^{j}+\left.\delta u^{j}\right|^{i}\right)\right]\right|_{j}\right\} \mathrm{d} \Omega}_{I_{3}}+ \\
& +\underbrace{\int_{0} \oint_{B} G \delta\left|\frac{d S^{\prime}}{d S}\right| \mathrm{d} S \mathrm{~d} t}_{I_{4}}+\underbrace{\int_{\Omega} \theta\left[\delta\left(J \beta_{q^{\prime}}^{k}\right) u^{q^{\prime}}\right]_{, k} \mathrm{~d} \Omega}_{I_{5}}+ \\
& -\underbrace{\int_{\Omega} \psi_{r^{\prime}}\left\{\partial_{o}\left(\delta J u^{r^{\prime}}\right)+\left[\delta\left(J \beta_{n^{\prime}}^{j}\right)\left(u^{r^{\prime}} u^{n^{\prime}}+g^{r^{\prime} n^{\prime}} p v-\nu u_{, p^{\prime}}^{r^{\prime}} g^{p^{\prime} n^{\prime}}\right)\right]_{, j}\right\} \mathrm{d} \Omega}_{I_{6}}
\end{aligned}
$$

Na primeira integral, $I_{1}$, o símbolo $\mathcal{F}^{k}$ representa as componentes da força resultante que fluído exerce sobre uma dada superfície (na direção normal $\mathbf{n}$ ): $\mathcal{F}^{k}=\tau^{k j} n_{j} \Rightarrow \delta \mathcal{F}^{k}=\delta \tau^{k j} n_{j}$. 
É importante ressaltar que a característica mais importante da equação 4.33 é que as integrais podem ser dividas em dois grupos: Aquelas que envolvem variações físicas $\left\{I_{1}, I_{2}, I_{3}\right\}$; e aquelas que consideram variações métricas $\left\{I_{4}, I_{5}, I_{6}\right\}$. O último grupo foi mantido em sua formulação original, como parte do gradiente de sensibilidade. Enquanto o primeiro grupo dará origem ao problema adjunto.

Na realidade, a idéia básica que método adjunto apresenta é a tentativa de eliminar as variações físicas do escoamento do cálculo do gradiente de sensibilidade. Os meios para atingir esse objetivo são o teorema de Gauss e a idéia de tranferir os operadores diferenciais que atuavam nas variáveis do escoamento para as variáveis adjuntas, como indicadade nas equações 4.12 e 4.13. Todo o procedimento supõe que todas as variavéis físicas são contínuas e diferenciáveis no domínio $\Omega$. É esperado que os campos de pressão e velocidade possuam este comportamento, dadas as condições do escoamento consideradas.

Dado o número de termos da equação 4.33 que terão a aplicação do teorema de Gauss, cada um será considerado separadamente. A primeira integral, $I_{1}$, é sobre a superfície do corpo ao longo do tempo e será mantida sob esta forma. A primeira integral de volume aparece em $I_{2}$ e para resolve-la, juntamente com as outras integrais, será definida uma notação abreviada do elemento de volume no espaço transformado, baseado na equação 4.27 :

$$
\mathrm{d} \Omega=\mathrm{d} V \mathrm{~d} t=J \mathrm{~d} \xi^{1} \mathrm{~d} \xi^{2} \mathrm{~d} \xi^{3} \mathrm{~d} t \equiv J \mathrm{~d} \bar{\xi} \mathrm{d} t
$$

Sendo o símbolo $d \bar{\xi}$ o elemento de volume no espaço transformado. O funcional $I_{2}$ pode ser escrito como sendo:

$$
\left.\int_{\Omega} \Theta \delta u^{k}\right|_{k} \mathrm{~d} \bar{\xi} \mathrm{d} t=\int_{0 \partial \mathcal{D}}^{T} \oint_{\Omega} \Theta \delta u^{k} n_{k} \mathrm{~d} S \mathrm{~d} t-\left.\int_{\Omega} \delta u^{k} \Theta\right|_{k} \mathrm{~d} \bar{\xi} \mathrm{d} t
$$

Onde o valor de $\Theta \equiv J \theta$ foi definido.

O primeiro termo de $I_{3}$ envolve uma derivada no tempo. Logo, a idéia será inverter a ordem de integração, o contrário do que é feito no teorema de Gauss. Obviamente, esta medida depende que as variáveis do escoamento sejam contínuas e suaves em $\Omega$

$$
\int_{\Omega} \varphi_{i} \partial_{o} \delta u^{i} \mathrm{~d} \bar{\xi} \mathrm{d} t=\left[\int_{\mathcal{D}} \varphi_{i} \delta u^{i} \mathrm{~d} \bar{\xi}\right]_{0}^{T}-\int_{\Omega} \delta u^{i} \partial_{o} \varphi_{i} \mathrm{~d} \bar{\xi} \mathrm{d} t
$$


Onde $\varphi_{i} \equiv J \psi_{i}$ foi definido.

O segundo e terceiro termos de $I_{3}$ serão tratados juntos, utilizando a equação da continuidade (Eq. 4.30), onde pode-se escrever:

$$
\begin{aligned}
\int_{\Omega} \varphi_{i}\left(\left.u^{j} \delta u^{i}\right|_{j}+u^{i}{ }_{j} \delta u^{j}\right) \mathrm{d} \bar{\xi} \mathrm{d} t & =\int_{0 \partial \mathcal{D}}^{T} \oint_{i} \varphi^{j} \delta u^{i} n_{j} \mathrm{~d} S \mathrm{~d} t-\left.\int_{\Omega} u^{j} \delta u^{i} \varphi_{i}\right|_{j}-\left.\varphi_{i} u^{i}\right|_{j} \delta u^{j} \mathrm{~d} \bar{\xi} \mathrm{d} t \\
& =\int_{0 \partial \mathcal{D}} \oint_{i} \varphi_{i} u^{j} \delta u^{i} n_{j} \mathrm{~d} S \mathrm{~d} t-\int_{\Omega} \delta u^{i}\left(\left.u^{j} \varphi_{i}\right|_{j}-\left.\varphi_{j} u^{j}\right|_{i}\right) \mathrm{d} \bar{\xi} \mathrm{d} t
\end{aligned}
$$

Apenas o primeiro termo foi integrado por partes. O quarto termo em $I_{3}$ é facilmente obtido:

$$
\int_{\Omega} \varphi_{i}\left(g^{i j} v \delta p\right) \mid{ }_{j} \mathrm{~d} \bar{\xi} \mathrm{d} t=\int_{0 \partial \mathcal{D}}^{T} \oint_{\Omega} v \delta p \varphi^{j} n_{j} \mathrm{~d} S \mathrm{~d} t-\int_{\Omega} v \delta p \varphi^{j}{ }_{j} \mathrm{~d} \bar{\xi} \mathrm{d} t
$$

Acima, usou-se o fato de que a variação do tensor métrico é nula.

Os dois últimos termos de $I_{3}$ necessitam um pouco mais de cuidado em sua manipulação. Eles são diferente dos anteriores pelo fato de necessitarem ser integrados por partes duas vezes. Retirando $\nu$ por um momento, obtém-se:

$$
\begin{aligned}
\left.\int_{\Omega} \varphi_{i}\left(\left.\delta u^{i}\right|^{j}+\left.\delta u^{j}\right|^{i}\right)\right|_{j} \mathrm{~d} \bar{\xi} \mathrm{d} t & =\int_{0 \partial \mathcal{D}}^{T} \oint_{i} \varphi_{i}\left(\left.\delta u^{i}\right|^{j}+\left.\delta u^{j}\right|^{i}\right) n_{j} \mathrm{~d} S \mathrm{~d} t-\left.\int_{\Omega} \varphi_{i}\right|_{j}\left(\left.\delta u^{i}\right|^{j}+\left.\delta u^{j}\right|^{i}\right) \mathrm{d} \bar{\xi} \mathrm{d} t \\
& =\int_{0 \partial \mathcal{D}}^{T}\left[\varphi_{i}\left(\left.\delta u^{i}\right|^{j}+\left.\delta u^{j}\right|^{i}\right) n_{j}-\left.\varphi_{i}\right|_{j}\left(\delta u^{i} n^{j}+\delta u^{j} n^{i}\right)\right] \mathrm{d} S \mathrm{~d} t+ \\
& +\int_{\Omega}\left(\left.\left.\delta u^{i} \varphi_{i}\right|_{j}\right|^{j}+\left.\left.\delta u^{j} \varphi_{i}\right|_{j}\right|^{i}\right) \mathrm{d} \bar{\xi} \mathrm{d} t
\end{aligned}
$$

Então, multiplicando a equação 4.39 por $\nu$, considerando a derivação $\delta \tau^{i j}$ da equação 4.31 e fazendo $\left.\varphi^{i}\right|_{i}=\left.\left(\varphi_{j} g^{j i}\right)\right|_{i}=\left.\varphi_{j}\right|^{j}$, obtém-se:

$$
\int_{\Omega} \frac{\left.\varphi_{i} \delta \tau^{i j}\right|_{j}}{\rho} \mathrm{d} \bar{\xi} \mathrm{d} t=\int_{0 \partial \mathcal{D}}^{T} \frac{\varphi_{i} \delta \mathcal{F}^{i}}{\rho}-\nu \delta u^{i}\left(\left.\varphi_{i}\right|_{j}+\left.\varphi_{j}\right|_{i}\right) n^{j} \mathrm{~d} S \mathrm{~d} t+\int_{\Omega} \delta u^{i} \nu\left(\left.\varphi_{i}\right|^{j}+\left.\varphi^{j}\right|_{i}\right){ }_{j} \mathrm{~d} \bar{\xi} \mathrm{d} t
$$


A equação 4.40 acima deixa claro a razão pela qual manteve-se a expressão completa de $\delta \tau^{i j}$ na variação física da equação de Navier-Stokes (Eq. 4.30). O propósito era manter aquela variação completa na integral de superfície, obtendo assim o termo $\delta \mathcal{F}^{i}=\delta \tau^{i j} n_{j}$. Após isso, o termo será combinado com o mesmo termo da integral sobre a superfície do corpo, de modo a obter-se a condição de contorno correspondente

É interessante notar que as integrais de superfície nas equações 4.35, 4.37-4.40 e o termo entre colchetes na equação 4.36, compõem um concomitante bilinear, que darão origem ao problema de condição de contorno adjunta, discutida abaixo. Finalmente, substituindo os resultados das manipulações matemáticas das equações 4.35-4.40 na equação 4.33, obtém-se:

$$
\begin{aligned}
T \delta I & =\int_{\Omega}\left\{\left.\varphi^{j}\right|_{j} v \delta p+\left[\partial_{o} \varphi_{i}+\left.u^{j} \varphi_{i}\right|_{j}-\left.\varphi_{j} u^{j}\right|_{i}-\left.\Theta\right|_{i}+\left.\nu\left(\left.\varphi_{i}\right|^{j}+\left.\varphi^{j}\right|_{i}\right)\right|_{j}\right] \delta u^{i}\right\} \mathrm{d} \bar{\xi} \mathrm{d} t+ \\
& -\int_{0 \partial \mathcal{D}}^{T}\left\{\left[-\Theta n_{i}+u^{j} \varphi_{i} n_{j}+\nu\left(\left.\varphi_{i}\right|_{j}+\left.\varphi_{j}\right|_{i}\right) n^{j}\right] \delta u^{i}+v\left(\delta p n^{i}-\delta \mathcal{F}^{i}\right) \varphi_{i}\right\} \mathrm{d} S \mathrm{~d} t+ \\
& \left.-\int_{\mathcal{D}} \varphi_{i} \delta u^{i} \mathrm{~d} V\right]_{0}^{T}+\int_{0} \oint_{B}\left[\frac{\partial G}{\partial p} \delta p+\frac{\partial G}{\partial \mathcal{F}^{k}} \delta \mathcal{F}^{k}\right]\left|\frac{\mathrm{d} S^{\prime}}{\mathrm{d} S}\right| \mathrm{d} S \mathrm{~d} t+ \\
& +\int_{0}^{T} \oint_{B} G \delta\left|\frac{\mathrm{d} S^{\prime}}{\mathrm{d} S}\right| \mathrm{d} S \mathrm{~d} t-\int_{\Omega} \frac{-\Theta}{J}\left[\delta\left(J \beta_{q^{\prime}}^{k}\right) u^{q^{\prime}}\right]_{, k} \mathrm{~d} \Omega+ \\
& -\int_{\Omega} \frac{\varphi_{r^{\prime}}}{J}\left\{\partial_{o}\left(\delta J u^{r^{\prime}}\right)+\left[\delta\left(J \beta_{n^{\prime}}^{j}\right)\left(u^{r^{\prime}} u^{n^{\prime}}+g^{r^{\prime} n^{\prime}} p v-\nu u_{, p^{\prime}}^{r^{\prime}} g^{p^{\prime} n^{\prime}}\right)\right], j\right\} \mathrm{d} \Omega
\end{aligned}
$$

Todas as variações físicas que aparecem na equação 4.41 são realizáveis porque satisfazem as equações de Navier-Stokes.

Como mencionado anteriormente, o problema adjunto é construído de tal forma a eliminar as variações físicas do escoamento no cálculo do gradiente de sensibilidade. Na equação acima, estes termos correspondem as primeiras quatro integrais. Naturalmente, essas integrais com as condições de contorno e de tempo adequadas, podem zerar esses termos, tornando o gradiente de sensibilidade independente das variações do escoamento. Esta é a única justificativa para todas os procedimentos matemáticos apresentados acima.

A primeira integral na equação 4.41 é sobre todo o domínio do escoamento e 
envolve duas variações arbitrárias, $\delta p$ e $\delta \mathbf{u}$. Uma forma de levar esta integral a zero é impor que os termos que multiplicam as variações sejam nulo. Isto implica que as variáveis adjuntas $\varphi$ e $\Theta$ deverão garantir essa condição, assim:

$$
\left\{\begin{array}{r}
\left.\varphi^{j}\right|_{j}=0 \\
\underline{\partial_{o} \varphi_{i}}+\left.u^{j} \varphi_{i}\right|_{j}-\left.\varphi_{j} u^{j}\right|_{i}-\left.\Theta\right|_{i}+\underline{\left.\left.\nu \varphi_{i}\right|^{j}\right|_{j}}=0
\end{array}\right.
$$

Onde o último termo do LHS da segunda equação foi simplificado em relação sua formulação original na equação 4.41, por conta da primeira equação do sistema. Adicionalmente, a derivada no tempo e o termo difusivo na segunda equação merecem destaque na análise por um importante motivo: Eles possuem o mesmo sinal e isto implica que o termo difusivo não se comportará como tal. Este termo acaba amplificando as pertubações na solução adjunta, causando instabilidade na solução numérica (BARKLEY; BLACKBURN; SHERWIN, 2002).

A solução para este problema é bem conhecida da literatura (BARKLEY; BLACKBURN; SHERWIN, 2002), e se caracteriza pela integração reversa no tempo. As equações serão integradas a partir das condições finais do escoamento, voltando no tempo até a situação inicial. Felizmente, este procedimento é totalmente conscistente com as condições de tempo adjuntas, como pode ser visto abaixo na equação 4.43. O tempo reverso será calculado simplesmente definindo uma variável adjunta de tempo:

$$
t^{*} \equiv T-t \Rightarrow \mathrm{d} t^{*}=-\mathrm{d} t \quad ; \quad\left\{\begin{array}{l}
t=0 \Rightarrow t^{*}=T \\
t=T \Rightarrow t^{*}=0
\end{array}\right.
$$

Com sua notação simplificada como sendo: $\partial_{o *} \equiv \partial\left(\right.$ ) $/ \partial t^{*}$.

Substituindo esta definição na derivação correspondente (Eq. 4.42), leva-se ao seguinte resultado:

$$
\left\{\begin{array}{r}
\left.\varphi^{j}\right|_{j}=0 \\
\partial_{o *} \varphi_{i}-\left.u^{j} \varphi_{i}\right|_{j}+\left.\varphi_{j} u^{j}\right|_{i}+\left.\Theta\right|_{i}-\left.\left.\nu \varphi_{i}\right|^{j}\right|_{j}=0
\end{array}\right.
$$

Este resultado corresponde as equações de Navier-Stokes adjuntas, aplicadas a escoamentos incompressíveis e isotérmicos. A primeira coisa que se observa na equação 4.44 é que ela é linear, uma vez que desde que todos os coeficientes são independentes das incógnitas $(\boldsymbol{\varphi}, \Theta)$.

Adicionalmente, comparando a equação 4.44 com a forma original das equa- 
ções de Navier-Stokes. percebe-se que ela possui a mesma estrutura, sendo ainda mais semelhante com a forma linearizada (Equação 4.30). As diferenças ocorrem no termo advectivo e no sinal dos operadores de primeira ordem. No entanto, os temos temporais e os mecanismos difusivos possuem semelhanças entre eles - e são estes termos que garantem a característica destas esquações diferenciais (CARRIER; PEARSON, 1988).

Como é conhecido das equações de Navier-Stokes, a equação de energia e quantidade de movimento são parabólicas no domínio do tempo e se tornam elípticas em regime permanente, enquanto a equação da continuidade é hiperbólica (HIRSCH, 1994a). A característica híbrida vem do fato de ser um escoamento incompressível e, devido sua semelhança, as equações adjuntas (4.44) serão da mesma forma. Todavia esta característica define a forma matemática das condições de contorno (MORSE; FESHBACH, 1953a; MORSE; FESHBACH, 1953b). Por outro lado, a física do problema das condições de contorno virá na segunda, terceira e quarta integrais da equação 4.41 .

\subsection{Condições de Contorno Adjuntas}

As condições temporais e de contorno da equação (4.44) devem satisfazer dois requisitos importantes: As primeiras integrais presentes na equação (4.41) devem ser nulas; e o problema adjunto deve ser condicionado, de tal forma a manter as características semelhantes ao problema do escoamento.

É apropriado citar aqui algumas observações presentes em (HIRSCH, 1994a) sobre as condições de contorno das equações de Navier-Stokes. O autor afirma que apesar das propriedades do problema de contorno serem bem conhecidas, não há regra universal para se obter um problema bem posto. Entretanto, é certo que as condições de contorno das equações de Navier-Stokes recuperam as equações de Euller, no caso de não ser considerada a viscosidade.

É sabido que alguma informação de Cauchy deve deixar de ser especificada na condição de saída, devido ao caráter hiperbólico da equação da continuidade. Para escoamentos não viscosos, somente uma condição será imposta para a saída. Logo, em vista das informações acima, a referência estabelece que condições viscosas adicionais deverão conter no máximo as derivadas de primeira ordem na direção do escoamento (HIRSCH, 1994a).

Apesar dessas observações serem aparentemente genéricas, elas são importantes para uma melhor compreensão do problema da condição de contorno adjunta, 
principalmente neste caso, onde não se pode usar os conhecimentos físicos para balizar as hipóteses utilizadas.

Analisando mais profundamente, pode-se observar que a segunda e terceira integrais na equação 4.41 representam o produto tensorial entre as variáveis adjuntas e as variações físicas, onde esta última é governada pelas condições de contorno do escoamento. Assim, as condições de contorno linearizadas são introduzidas nas integrais a fim de garantir a realizibilidade do problema. Portanto, as expressões resultantes serão utilizadas para a derivação das condições de contorno adjuntas, que estarão de acordo com as características das PDEs. A quarta integral na equação 4.41 é utilizada somente para se obter a condição de contorno de parede sólida.

\subsubsection{Condições Temporais}

Nas aplicações que interessam a este trabalho, as condições temporais impõem valores conhecidos para as variáveis de estado $\mathbf{u}$ para $t=0$. Como resultado, temse que:

$$
\left.\delta \mathbf{u}\right|_{t=0}=0
$$

Por outro lado, a terceira integral da equação 4.41 pode ser expandida para:

$$
-\left[\int_{\mathcal{D}} \varphi_{i} \delta u^{i} \mathrm{~d} V\right]_{0}^{T}=\left(\int_{\mathcal{D}} \varphi_{i} \delta u^{i} \mathrm{~d} V\right)_{t=0}-\left(\int_{\mathcal{D}} \varphi_{i} \delta u^{i} \mathrm{~d} V\right)_{t=T}
$$

Onde a equação 4.45 provoca o desaparecimento do primeiro termo, independente dos valores $\varphi$ dados para $t=0$. A maneira mais direta de levar o segundo termo a zero é impondo uma condição final homogênea para esta variável:

$$
\left.\varphi\right|_{t=T}=0
$$

Enquanto $\delta \mathbf{u}$ permanece livre em $t=T$. Em termos da variável adjunta de tempo (Eq. 4.43), estas condições ficam como sendo:

$$
\left.\boldsymbol{\varphi}\right|_{t^{*}=0}=0 \quad ;\left.\quad \delta \mathbf{u}\right|_{t^{*}=T}=0
$$

Enquanto $\delta \mathbf{u}$ é livre em $t^{*}=0$, e $\boldsymbol{\varphi}$ é livre em $t^{*}=T$.

As condições da equação 4.48 fazem a terceira integral da equação 4.41 desaparecer. Adicionalmente, elas são consistentes com as características da equação 
4.44 e são compatíveis com a operação reversa no tempo de acordo com a equação 4.43

\subsubsection{Formulação das Condições de Contorno}

As condições de contorno adjuntas são puxadas para tornar nula a segunda e quarta integrais da equação 4.41, que serão colocadas novamente a seguir, para melhor conveniência do leitor:

$$
\begin{aligned}
& -\int_{0 \partial \mathcal{D}}^{T}\left\{\left[-\Theta n_{i}+u^{j} \varphi_{i} n_{j}+\nu\left(\left.\varphi_{i}\right|_{j}+\left.\varphi_{j}\right|_{i}\right) n^{j}\right] \delta u^{i}+v\left(\delta p n^{i}-\delta \mathcal{F}^{i}\right) \varphi_{i}\right\} \mathrm{d} S \mathrm{~d} t+ \\
& +\int_{0} \oint_{B}\left[\frac{\partial G}{\partial p} \delta p+\frac{\partial G}{\partial \mathcal{F}^{k}} \delta \mathcal{F}^{k}\right]\left|\frac{\mathrm{d} S^{\prime}}{\mathrm{d} S}\right| \mathrm{d} S \mathrm{~d} t
\end{aligned}
$$

A quarta integral é sobre a superfície do corpo $B$ e portanto é a única que afeta a condição de contorno de parede. As condições de contorno relevantes para este trabalho serão apresentadas a seguir:

\subsubsection{Planos de Corte}

As variáveis do escoamento são assumidas como contínuas e suaves através dos planos de corte, que são fronteiras idealizadas não-físicas. Essa hipótese implica que a solução do escoamento e sua componente linearizada são impostas como condições periódicas sobre tais fronteiras.

Com a imposição das condições de contorno periódicas para as variáveis adjuntas $(\varphi, \Theta)$, as integrais de superfícies tornam-se idênticas nos dois lados do corte. A direção destas variáveis será dada pela orientação do vetor normal e o resultado será que estas integrais de superfície irão se cancelar em toda a fronteira, sendo portanto eliminados.

Por outro lado, as condições periódicas são conhecidas por serem consistentes e bem condicionadas nas equações que governam o escoamento. Logo, esta consistência deve ser mantida para a equação linearizada de Navier-Stokes e para o problema adjunto por compartilharem das mesmas características.

$$
\left.(\delta \mathbf{u}, p)\right|_{c u t}=\text { periódico } \quad ;\left.\quad(\delta \boldsymbol{\varphi}, \Theta)\right|_{c u t}=\text { periódico }
$$




\subsubsection{Condição de Parede}

Para a condição de parede sólida, a condição de não escorregamento é imposta nas equações que governam o escoamento. Obviamente isto leva que a velocidade na superfície sob esta condição será nula:

$$
\left.\mathbf{u}\right|_{\text {wall }}=\left.\delta \mathbf{u}\right|_{\text {wall }}=0
$$

A primeira integral na equação 4.49 é aplicada sobre a superfície do corpo $B$ e implica que o termo correspondente é cancelado, independente do seu coeficiente. Combinando a segunda integral, com a esta equação, é obtido a condição de contorno de parede:

$$
\int_{0}^{T} \oint_{B}\left[\left(\frac{\partial G}{\partial p}\left|\frac{\mathrm{d} S^{\prime}}{\mathrm{d} S}\right|-v \varphi_{i} n^{i}\right) \delta p+\left(\frac{\partial G}{\partial \mathcal{F}^{i}}\left|\frac{\mathrm{d} S^{\prime}}{\mathrm{d} S}\right|+v \varphi_{i}\right) \delta \mathcal{F}^{i}\right] \mathrm{d} S \mathrm{~d} t
$$

Considerando que as variações de $\delta p$ e $\delta \mathcal{F}^{i}$ são arbritárias e independentes, o único meio de tornar este termo nulo é impondo as condições da solução adjunta, em fronteiras sólidas:

$$
\left\{\begin{aligned}
\left.\varphi^{i} n_{i}\right|_{w} & =\left(\rho \frac{\partial G}{\partial p}\left|\frac{\mathrm{d} S^{\prime}}{\mathrm{d} S}\right|\right)_{\text {wall }} \\
-\left.\varphi^{i}\right|_{w} & =\left(\rho \frac{\partial G}{\partial \mathcal{F}^{i}}\left|\frac{\mathrm{d} S^{\prime}}{\mathrm{d} S}\right|\right)_{\text {wall }}
\end{aligned}\right.
$$

Estas relações claramente colocam uma restrição na forma das medidas de mérito (Eq.4.21). Para manter-se a consistência, $G$ deverá satisfazer a relaçao:

$$
\left.\frac{\partial G}{\partial p}\right|_{\text {wall }}=-\left.n^{i} \frac{\partial G}{\partial \mathcal{F}^{i}}\right|_{\text {wall }}
$$

Como resultado, qualquer uma das equações (4.53) é adequada para as condições de contorno adjuntas, desde que esteja acompanhadas pela equação 4.54. Além disso, cada uma das condições nas equações 4.53 é do tipo Dirichlet, e restringem o mesmo número de variáveis $\varphi^{k}$ que a condição de aderência restringe em $u^{k}$. A variável $\Theta$ é determinada pela solução da equação adjunta como a pressão é determinada na solução do escoamento. Portanto, a condição de contorno de parede adjunta é totalmente consistente com o problema.

Como ilustração de o quão restritiva esta condição pode ser, toma-se um caso particular onde a função $G$ é a própria projeção de força que o fluído exerce sobre 
o corpo em uma dada direção e:

$$
G(\mathbf{n} \cdot \boldsymbol{\sigma} \cdot \mathbf{e})=\mathcal{F}^{k} e_{k}-p n^{k} e_{k}
$$

Aplicando a condição 4.54 na equação acima, chega-se ao seguinte resultado:

$$
\left.\frac{\partial G}{\partial p}\right|_{\text {wall }}=-\left.n^{i} \frac{\partial G}{\partial \mathcal{F}^{i}}\right|_{\text {wall }}=-n^{k} e_{k}
$$

Enquanto as equações 4.53 formam a seguinte condição de parede:

$$
\left.\varphi_{k}\right|_{w}=-\left(\rho e_{k}\left|\frac{\mathrm{d} S^{\prime}}{\mathrm{d} S}\right|\right)_{\text {wall }}
$$

O exemplo apresenta que a restrição 4.54 parece impor em $G$ a mesma relação entre $p g^{i j}$ e $\tau^{i j}$ que existe no tensor das tensões $\sigma^{i j}$.

\subsubsection{Condição de Entrada}

Para dedução das equações será admitido que o vetor normal está orientado para fora do domínio, logo a seção de entrada do domínio terá a seguinte condição: $\mathbf{u} \cdot \mathbf{n}<0$.

O vetor velocidade é imposto como um perfil não nulo na fronteira de entrada e consequentemente, dará a seguinte condição linearizada similar ao caso anterior:

$$
\left.\delta \mathbf{u}\right|_{\text {in }}=0
$$

Neste caso, a condição de entrada empregada na equação 4.49 será um pouco diferente da condição de parede. Aplicando a equação 4.58 nela, obtém-se o seguinte resultado:

$$
\int_{0 \partial \mathcal{D}}^{T} \oint v\left(\delta \mathcal{F}^{i} \varphi_{i}-\delta p n^{i} \varphi_{i}\right) \mathrm{d} S \mathrm{~d} t
$$

Novamente neste caso, as variações físicas $\delta \mathcal{F}^{i}$ e $\delta p$ serão arbitrárias e independentes. Logo, a única maneira de levar a integral a zero é assumir que:

$$
\left\{\begin{array}{c}
\varphi_{i}=0 \\
n^{i} \varphi_{i}=0
\end{array}\right.
$$


A segunda equação é suficiente apenas para impor que $\varphi \perp \mathbf{n}$. Entretanto, a primeira equação a sobrescreve, garantindo que a integral da equação 4.49 se torne nula, com o mesmo efeito na equação 4.41. Como resultado, as condições de contorno homogêneas de Dirichlet são impostas na condição de entrada como sendo $\varphi$.

$$
\left.\varphi\right|_{i n}=0
$$

Como já visto anteriormente, a condição de entrada adjunta é muito similar a $\mathbf{u}$ e $\delta \mathbf{u}$ que são impostas nas equações que governam o escoamento. Por outro lado, $\Theta$ é deixado livre na fronteira, da mesma forma que $p$ é na solução do escoamento. Devido ao fato que o as equações do escoamento e as adjuntas compartilharem das mesmas características matemáticas, a equação 4.61 deverá se manter consistente como as as outras deduzidas anteriormente.

\subsubsection{Condição de Saída}

Devido a orientação do vetor normal na condição de entrada (para fora do domínio), a seção de saída será a porção do domínio externo onde u.n $\geq 0$.

De acordo com o que foi proposto em (BARKLEY; BLACKBURN; SHERWIN, 2002), a condição de saída deve ser imposta com pressão constante e a componente da velocidade na direção normal nula. Pelo fato de ser um escalar, a condição de pressão não possui nenhuma variação métrica, necessitando apenas que sua variação física seja nula $(\delta p=0)$. Entretanto, a derivada da condição de velocidade certamente terá variações métricas.

$$
\begin{aligned}
& u^{i^{\prime}}\left|j^{\prime} n_{j^{\prime}}=\beta_{k}^{i^{\prime}} u^{k}\right|^{r^{\prime}} \partial_{r^{\prime}}^{j^{\prime}} n_{j^{\prime}}=0 \\
& =\left.\beta_{k}^{i^{\prime}} u^{k}\right|^{r^{\prime}} \beta_{r^{\prime}}^{p} \beta_{p}^{j^{\prime}} n_{j^{\prime}}=\left.\beta_{k}^{i^{\prime}} u^{k}\right|^{p} n^{p} \\
& \left.u^{k}\right|^{p} n_{p}=0 \\
& \left.\partial u^{i^{\prime}}\right|^{j^{\prime}} n_{j^{\prime}}=\left.\partial \beta_{k}^{i^{\prime}} u^{k}\right|^{p} n_{p}+\left.\beta_{k}^{i^{\prime}} \partial u^{k}\right|^{p} n_{p}=0 \\
& \left.\partial u^{k}\right|^{p} n_{p}=0
\end{aligned}
$$

Estas condições correspondem as seguintes restrições das equações linearizadas: 


$$
\begin{cases}\delta \mu u^{i} \mid{ }^{j} n_{j} & =0 \\ \delta p & =0\end{cases}
$$

A primeira equação acima implica que $\delta \mathcal{F}^{i}=\tau^{i j} n_{j}=\left.\mu \delta u^{j}\right|^{i} n_{j}$. Adicionalmente, a equação 4.62 foi multiplicada por $\mu$ na equação 4.63 para indicar que ela deverá ser imposta em um escoamento viscoso. Se admitirmos $\nu \delta u^{i} \mid{ }^{j} n_{j}=0$, recupera-se a condição de Euller no limite em que a viscosidade desaparece $(\delta p=0)$. Utilizando este resultado e introduzindo as relações na equação 4.49, temos que:

$$
\int_{0 \partial \mathcal{D}}^{T}\left[\Theta n_{i}-u^{j} \varphi_{i} n_{j}-\nu\left(\left.\varphi_{i}\right|_{j}+\left.\varphi_{j}\right|_{i}\right) n^{j}\right] \delta u^{i} \mathrm{~d} S \mathrm{~d} t+\left.\int_{0 \partial \mathcal{D}}^{T} \oint_{j} \nu n_{j} \delta u^{j}\right|^{i} \varphi_{i} \mathrm{~d} S \mathrm{~d} t
$$

Onde as variações $\delta u^{i}$ e $\left.\delta n_{j} u^{j}\right|^{i}$ são assumidas totalmente independentes $a$ priori como uma tentativa de levar a integral de superfície a zero. O problema gerado com essa hipótese é que a mesma leva a seis condições de saída, sobreespecificando as equações adjuntas.

Como forma de contornar o problema, a segunda integral da equação 4.64 será considerada separadamente e buscará encontrar uma relação entre $\delta u^{i}$ e $\left.\delta n_{j} u^{j}\right|^{i}$. Por fim, será utilizado o teorema de Gauss. A integral é resolvida na superfície do volume de controle com espessura infinitesimal, $V_{o}$, que é definida sobre a condição de saída - a constante $\nu$ é colocada para fora da integral.

$$
\begin{array}{r}
\left.\int_{0 \partial V_{o}}^{T} n_{j} \delta u^{j}\right|^{i} \varphi_{i} \mathrm{~d} S \mathrm{~d} t=\left.\int_{0}^{T} \int_{V_{o}}\left(\left.\delta u^{j}\right|^{i} \varphi_{i}\right)\right|_{j} \mathrm{~d} \bar{\xi} \mathrm{d} t= \\
=\int_{0}^{T} \int_{V_{o}}\left(\left.\left.\delta u^{j}\right|_{j}\right|^{i} \varphi_{i}+\left.\left.\partial u^{j}\right|^{i} \varphi_{i}\right|_{j}\right) \mathrm{d} \bar{\xi} \mathrm{d} t \\
\left.\int_{0 \partial V_{o}} n_{j} \delta u^{j}\right|^{i} \varphi_{i} \mathrm{~d} S \mathrm{~d} t=\left.\left.\int_{0}^{T} \int_{V_{o}} \delta u^{j}\right|^{i} \varphi_{i}\right|_{j} \mathrm{~d} \bar{\xi} \mathrm{d} t= \\
=\int_{0}^{T} \int_{V_{o}}\left[\left.\left(\left.\varphi_{i}\right|_{j} \partial u^{j}\right)\right|^{i}-\left.\left.\varphi_{i}\right|^{i}\right|_{j} \partial u^{j}\right] \mathrm{d} \bar{\xi} \mathrm{d} t
\end{array}
$$




$$
\left.\int_{0 \partial V_{o}}^{T} \oint_{j} \delta u^{j}\right|^{i} \varphi_{i} \mathrm{~d} S \mathrm{~d} t=\left.\int_{0}^{T} \int_{V_{o}} \varphi_{i}\right|_{j} \partial u^{j} n^{i} \mathrm{~d} S \mathrm{~d} t
$$

Onde a ordem da segunda derivada covariante foi mudada, como discutido no apêndice $\mathrm{A}$, também foi usada a propriedade soleinodal tanto em $\delta u^{j}$ como em $\varphi_{i}$. Vale a pena ressaltar que a equação 4.69 é uma propriedade de $\left(\left.n_{j} \partial u^{j}\right|^{i} \varphi_{i}\right)$, cujo termo $\left(\varphi_{i} \partial u^{i} \mid{ }^{j} n_{j}\right)$ não compartilha, e isto é facilmente demonstrado quando se aplica o mesmo procedimento.

Conclui-se então que $\partial u^{i}{ }^{j} n_{j}$ é realmente a única parte de $\delta \mathcal{F}^{i}$ que é independente de $\partial u^{i}$. Enfim, controlando $\delta u^{i}$ em uma dada superfície, ele poderá variar independente só na direção normal à mesma. Entretanto a variação da componente normal foi imposta como sendo nula. Substituindo a equação 4.69 na equação 4.66, temos que:

$$
\begin{array}{r}
\int_{0 \partial \mathcal{D}}^{T}\left[\Theta n_{i}-u^{j} \varphi_{i} n_{j}-\nu\left(\left.\varphi_{i}\right|_{j}+\left.\varphi_{j}\right|_{i}\right) n^{j}\right] \partial u^{i}+\left.\nu \varphi_{j}\right|_{i} \partial u^{i} n^{j} \mathrm{~d} S \mathrm{~d} t= \\
=\int_{0 \partial \mathcal{D}} \oint_{T}\left[\Theta n_{i}-u^{j} \varphi_{i} n_{j}-\left.\nu \varphi_{i}\right|_{j} n^{j}\right] \partial u^{i} \mathrm{~d} S \mathrm{~d} t
\end{array}
$$

Para levar a integral acima a zero a partir de um arbitrário $\delta u^{i}$, serão impostas a seguintes condições para as variáveis adjuntas:

$$
\left.\Theta n_{i}\right|_{\text {out }}=u^{j} n_{j} \varphi_{i}+\left.\nu \varphi_{i}\right|_{j} n^{j}
$$

A equação 4.72 representa três condições de contorno mistas. Assim, impõe um menor número de restrições sobre as variáveis adjuntas do que suas correspondentes do escoamento. Além disso, as equações são levemente diferentes das presentes em 4.63 e não se pode fazer uma simples analogia entre elas. Portanto, são necessários alguns testes adicionais para verificar se este problema está bem posto.

Entretanto há uma possível solução para essas diferenças entre as condições de contorno que pode ser considerada. Numa analogia com o problema físico, onde a pressão é imposta na seção de saída, pode-se tentar a pressão como condição 
de Dirichlet na solução do escoamento incompressível. Logo, pode-se testar a seguinte possibilidade:

$$
\left\{\begin{array}{l}
\Theta=0 \\
u^{j} n_{j} \varphi_{i}=-\left.\nu \varphi_{i}\right|_{j} n^{j}
\end{array}\right.
$$

\subsubsection{Planos de Simetria}

A condição de contorno que é imposta no plano de simetria juntamente com as suas variações físicas é dada por:

$$
\left\{\begin{aligned}
\left.u^{i} n_{i}\right|_{s y m}=0 & \left.\Rightarrow \delta u^{i} n_{i}\right|_{s y m}=0 \\
u^{i}\left|{ }_{j} n^{j}\right|_{s y m}=0 & \Rightarrow \delta u^{i}\left|{ }_{j} n^{j}\right|_{s y m}=0 \\
\left.\left.p\right|^{j} n_{j}\right|_{s y m}=0 & \Rightarrow \delta p\left|{ }^{j} n_{j}\right|_{\text {sym }}=0
\end{aligned}\right.
$$

Por ser uma superfície plana no espaço físico, conclui-se que o vetor normal é estritamente constante e com isso chega-se a:

$$
\begin{aligned}
\left.n_{i} u^{i}\right|_{j} & =n_{i} \beta_{r^{\prime}}^{i}\left(\beta_{n}^{r^{\prime}} u^{n}\right)_{, j} \\
& =n_{r^{\prime}}\left(u^{r^{\prime}}\right)_{, s^{\prime}} \beta_{j}^{s^{\prime}} \\
& =\left(n_{r^{\prime}} u^{r^{\prime}}\right)_{, s^{\prime}} \beta_{j}^{s^{\prime}} \\
\left.n_{i} u^{i}\right|_{j} & =0
\end{aligned}
$$

Onde foi utilizado o que foi deduzido na equação 4.74. A consequência direta da equação 4.75 é que $\left.n_{i} \delta u^{i}\right|_{j}=0$, com isso, a força que fluído exerce sobre o plano de simetria torna-se nula:

$$
\begin{aligned}
\mathcal{F}^{i} & =\tau^{i j} n_{j} \\
& =\mu\left(\left.u^{i}\right|^{j}+\left.u^{j}\right|^{i}\right) n_{j} \\
& =\left.\mu n_{j} u^{j}\right|^{i}=\left.\mu n_{j} u^{j}\right|_{k} g^{k j} \\
\mathcal{F}^{i} & =0
\end{aligned}
$$

Conforme era esperado, valendo o mesmo para a sua primeira variação $\delta \mathcal{F}^{i}=$ 0. 
Substituindo estes resultados na equação 4.49, obtém-se que:

$$
\left.-\int_{0 \partial \mathcal{D}}^{T} \oint_{\nu n^{j}}\left(\left.\varphi_{i}\right|_{j}+\left.\varphi_{j}\right|_{i}\right) \delta u^{i}+v n^{i} \varphi_{i} \delta p\right] \mathrm{d} S \mathrm{~d} t
$$

e então, chega-se ao seguinte equacionamento:

$$
\left\{\begin{aligned}
\left(\left.\varphi_{i}\right|_{j}+\left.\varphi_{j}\right|_{i}\right) n^{j} & =0 \\
n^{i} \varphi_{i} & =0
\end{aligned}\right.
$$

A segunda equação mostra que $\varphi \perp \mathbf{n}$. Seguindo o mesmo raciocínio que foi feito na equação 4.75, pode-se mostrar facilmente que:

$$
\left.n_{i} \varphi^{i}\right|_{j}=\left.n^{i} \varphi_{i}\right|_{j}=\left.0 \Rightarrow \varphi_{i}\right|_{j} n^{j}=0
$$

A equação da direita foi obtida através da combinação das anteriores com a equação 4.78 .

Assim como na primeira expressão da equação 4.78, define-se um volume de controle infinitesimal sobre o plano de simetria e aplicando o teorema de Gauss na mesma, pode-se mostrar que o tensor adjunto terá divergência nula naquele plano:

$$
\begin{array}{r}
\left.\left(\left.\varphi_{i}\right|_{j}+\left.\varphi_{j}\right|_{i}\right)\right|^{j}=0 \\
\left.\left.\varphi_{i}\right|_{j}\right|^{j}=0
\end{array}
$$

Onde a primeira equação adjunta foi utilizada.

Finalmente, projetando a segunda equação adjunta (Eq. 4.44) sobre a direção normal no plano de simetria e substituindo as equações 4.74 a 4.80 em seus respectivos termos, obtem-se que:

$$
\begin{aligned}
n^{i} \partial_{o *} \varphi_{i}-\left.u^{j} n^{i} \varphi_{i}\right|_{j}+\varphi_{j} u^{j}{ }_{i} n^{i}+\left.\Theta\left|{ }_{i} n^{i}-\nu n^{i} \varphi_{i}\right|^{j}\right|_{j} & =0 \\
\Theta{ }_{i} n^{i} & =0
\end{aligned}
$$

O resultado final destas derivações é que a equação 4.78 pode ser substituída por equações equivalentes que representam as condições de contorno adjuntas 
impostas no plano de simetria:

$$
\left\{\begin{array}{r}
\left.\varphi^{i} n_{i}\right|_{s y m}=0 \\
\varphi_{i}\left|{ }_{j} n^{j}\right|_{s y m}=0 \\
\Theta\left|{ }_{j} n^{j}\right|_{\text {sym }}=0
\end{array}\right.
$$

Estas condições são idênticas a aquelas que são impostas no campo do escoamento (Eq.4.74). Não apenas o plano de simetria do escoamento se comporta como um plano de simetria na solução adjunta, como também garante que a equação 4.82 fique totalmente compatível e consistente com o problema. Novamente, fica claro que as equações possuem as mesmas características. Assim, a equação 4.74 é bem condicionada e consistente no problema.

\subsubsection{Considerações finais sobre a Modelagem}

O conjunto de equações adjuntas é geral, quando se mantém sob a transformação de coordenadas. Portanto, é possível escreve-las na forma simbólica abaixo:

$$
\left\{\begin{aligned}
\partial_{o *} \boldsymbol{\varphi}-(\mathbf{u} \cdot \nabla) \boldsymbol{\varphi}+(\nabla \mathbf{u})^{T} \cdot \boldsymbol{\varphi}+\nabla \Theta-\nu \nabla^{2} \boldsymbol{\varphi} & =0 \\
\nabla \cdot \boldsymbol{\varphi} & =0
\end{aligned}\right.
$$

O equacionamento acima pode ser posto ao lado das equações originais de Navier-Stokes para comparação:

$$
\left\{\begin{aligned}
\partial_{o} \mathbf{u}+(\mathbf{u} \cdot \nabla) \mathbf{u}+v \nabla p-\nu \nabla^{2} \mathbf{u} & =0 \\
\nabla \cdot \mathbf{u} & =0
\end{aligned}\right.
$$

Como pode ser visto, as diferenças são evidentes nos mecanismos convectivos, mas o difusivo e o passo no tempo são os mesmos.

As condições de contorno adjuntas são resumidas na tabela 4.1 abaixo, onde elas são pareadas com as condições de contorno originais e linearizadas do escoamento.

A condição de contorno de parede impôs uma restrição no núcleo do funcional 
Tabela 4.1: Condições de Contorno do Escoamento e Adjuntas. Sendo $\varphi$ a magnitude do vetor de velocidade adjunta, $\varphi=\|\varphi\|$.

\begin{tabular}{llll}
\hline Contorno & Escoamento & Linearizada & Adjunta \\
\hline Tempo & $\left.\mathbf{u}\right|_{t=0}=\mathbf{u}_{o}$ & $\left.\delta \mathbf{u}\right|_{t=0}=0$ & $\left.\boldsymbol{\varphi}\right|_{t^{*}=0}=0$ \\
Planos de corte & periódica & periódica & periódica \\
Entrada & $\mathbf{u}$ fixado & $\delta \mathbf{u}=0$ & $\boldsymbol{\varphi}=0$ \\
Saída & $\frac{\partial \mathbf{u}}{\partial n}=0$ & $\frac{\partial \delta \mathbf{u}}{\partial n}=0$ & $\frac{\partial \varphi}{\partial n}=-\frac{(\mathbf{u} . \mathbf{n}) \varphi}{\nu}$ \\
& $p$ fixado & $\delta p=0$ & $\Theta=0$ \\
Parede & $\mathbf{u}=0$ & $\delta \mathbf{u}=0$ & $\boldsymbol{\varphi}=-\rho \frac{\partial G}{\partial \mathcal{F}}\left|\frac{\mathrm{d} S^{\prime}}{\mathrm{d} S}\right|$ \\
Plano de Simetria & $\mathbf{u} \cdot \mathbf{n}=0$ & $\delta \mathbf{u} \cdot \mathbf{n}=0$ & $\boldsymbol{\varphi} \cdot \mathbf{n}=0$ \\
& $\frac{\partial \mathbf{u}}{\partial n}=\frac{\partial p}{\partial n}=0$ & $\frac{\partial \delta \mathbf{u}}{\partial n}=\frac{\partial \delta p}{\partial n}=0$ & $\frac{\partial \boldsymbol{\varphi}}{\partial n}=\frac{\partial \Theta}{\partial n}=0$ \\
\hline
\end{tabular}

objetivo $G(\mathbf{n} \cdot \boldsymbol{\sigma} \cdot \mathbf{e})$, chegando a:

$$
\left.\frac{\partial G}{\partial p}\right|_{\text {wall }}=-\left.\frac{\partial G}{\partial \mathcal{F}} \cdot \mathbf{n}\right|_{\text {wall }}
$$

Tomando as variáveis adjuntas como sendo soluções das equações acima e substituindo elas na expressão do variacional aumentado (Eq.4.41), elas eliminarão todas as variações físicas desta expressão. Como resultado, a expressão de $\delta I$ se reduz a:

$$
\begin{aligned}
T \delta I & =\int_{0} \oint_{B} G \delta\left|\frac{d S^{\prime}}{d S}\right| \mathrm{d} S \mathrm{~d} t+\int_{\Omega} \frac{\Theta}{J}\left[\delta\left(J \beta_{q^{\prime}}^{k}\right) u^{q^{\prime}}\right]_{, k} \mathrm{~d} \Omega+ \\
& -\int_{\Omega} \frac{\varphi_{r^{\prime}}}{J}\left\{\partial_{o}\left(\delta J u^{r^{\prime}}\right)+\left[\delta\left(J \beta_{n^{\prime}}^{j}\right)\left(u^{r^{\prime}} u^{n^{\prime}}+g^{r^{\prime} n^{\prime}} p v-\nu u_{, p^{\prime}}^{r^{\prime}} g^{p^{\prime} n^{\prime}}\right)\right]_{, j}\right\} \mathrm{d} \Omega
\end{aligned}
$$

Esta equação pode ser usada para calcular o gradiente de sensibilidade sem a necessidade de um custo adicional de simulações do escoamento que outros métodos baseados no gradiente necessitam. Esta propriedade do método adjunto é independente do número de parâmetros de controle do projeto. Adicionalmente é importante lembrar que existe uma flexibilidade para mudar a medida de mérito de acordo com as diversas aplicações existentes na hidrodinâmica.

As equações adjuntas (4.83) são similiares as apresentadas por (BARKLEY; 
BLACKBURN; SHERWIN, 2002) com algumas diferenças nas condições de contorno pois há diferenças na natureza das aplicações dos trabalhos. 


\section{Resultados da Simulação ADJUNTA}

Esta seção irá descrever os resultados obtidos e os comentários pertinentes das simulações do método adjunto.

Os capítulos anteriores mostraram o desenvolvimento de toda formulação do método adjunto baseada nas equações de Navier Stokes para escoamento incompressível, com enfoque em suas condições de contorno. Apesar deste trabalho apresentar apenas a avaliação das sensibilidades geométricas, é importante ressaltar que a formulação adjunta é capaz de avaliar as sensibilidades não geométricas, mostrando a versatilidade do método.

Entretanto, o presente trabalho apresentará apenas os resultados preliminares das simulações adjuntas, que foram obtidas a partir do trabalho de validação que prosseguirá nos trabalhos posteriores a esta dissertação.

\subsection{Resultados Preliminares}

Como mencionado acima, a validação completa dos resultados das simulações adjuntas, bem como as primeiras estimativas do gradiente de sensibilidade não fazem parte do escopo deste trabalho.

Todo grupo de pesquisa está envolvido nos dois tipos de sensibilidades (geométrica e não-geométrica) e necessitou-se de uma atenção especial na formulação das condições de contorno, onde a maior parte dos estudos esteve concentrada. Como resultado, produziu-se o trabalho descrito em (VOLPE et al., 2012).

Após as análises, verificou-se que as duas sensibilidades usam as mesmas condições de contorno e que uma única programação do método adjunto seria necessária para atender os dois tipos de aplicações.

Como visto no capítulo 4.3, os dois códigos (NEKTAR e SEMTEX) são capazes de resolver o escoamento e obter a solução adjunta. Entretanto, o código 
SEMTEX possui uma melhor flexibilidade para alteração das soluções adjuntas, sendo vantajoso para a aplicação proposta neste trabalho. Com essa vantagem, é possível alterar a condição de contorno na saída do escoamento (equação 4.73) com poucas intervenções no código.

$$
\left\{\begin{array}{c}
\frac{\partial \varphi}{\partial n}=-\frac{(u \cdot n) \varphi}{v} \\
\Theta=0
\end{array}\right.
$$

Esta condição está implementada apenas no código SEMTEX, porém limitada a perfis de velocidade na direção normal (u.n) estacionários na seção de saída.

Tentou-se implementar a mesma solução no software NEKTAR. Infelizmente, não se obteve sucesso na alteração do código devido a grande complexidade do mesmo. Na busca de alternativas, encontrou-se no software SEMTEX a possibilidade dessa alteração sem grandes demandas de programação.

Como primeira tentativa de uso do código, procurou-se testar o mesmo caso apresentado como exemplo no software. O exemplo consiste em um escoamento bidimensional ao redor de um cilindro circular como visto na figura 5.3 a seguir. É importante ressaltar que neste caso, a malha computacional é bem menor que as malhas feitas para o NEKTAR, descritas no capítulo 2.

Entretanto, não houve preocupação com a precisão da solução do escoamento, buscando apenas verificar se era possível obter uma solução adjunta para o caso, com as condições de contorno propostas no desenvolvimento teórico.

O número de Reynolds foi configurado para o valor $R e=30$, bem abaixo dos valores das simulações do capítulo 3 , que estão na região onde o escoamento forma esteiras periódicas de vórtices. A justificativa deste valor é a garantia que o perfil da velocidade normal na região de saída do domínio externo seja estacionário. Assim, o conjunto de equações 5.1 é satisfeito pelo SEMTEX. Em relação ao grau do Polinômio interpolador, configurou-se o valor de 8, recomendado pelo pesquisador.

A medida de mérito é dada como sendo a variação da média do coeficiente de arrasto presente no conjunto de equações 1.2, que aplicada a equação 4.21, gera o seguinte resultado:

$$
I_{0}=\overline{C_{f}}=\frac{1}{q_{\infty} T} \int_{0}^{T} \int_{B} n \cdot \sigma \cdot e_{x} d S d t
$$


onde $\boldsymbol{e}_{\boldsymbol{x}}$ é paralelo a direçao do escoamento (ao longo do eixo x), e $\mathbf{B}$ representa o contorno do cilindro. As condições de contorno da tabela 4.1 foram impostas no escoamento e na solução adjunta. Em particular, a condição de contorno de parede pode ser obtida diretamente das equações 4.54-4.56,

$$
\varphi=-\rho e_{x}\left|\frac{d S^{\prime}}{d S}\right|_{B}
$$

e, obviamente, a equação 5.1 foi imposta como condição de contorno na saída da solução adjunta.

Felizmente não houve dificuldades para se obter uma solução adjunta convergida. Entretanto, uma análise mais aprofundada da condição de contorno de saída verificou que existia um erro numérico. Enquanto a pressão adjunta $\Theta$ ficou como uma condição de contorno de Dirichilet homogênea (como esperado), a precisão da velocidade adjunta $\varphi$ na condição de contorno mista se mostrou diferente, em outras palvras, na comparação entre os lados esquerdo e direito da equação 5.1, os valores se mostraram diferentes.
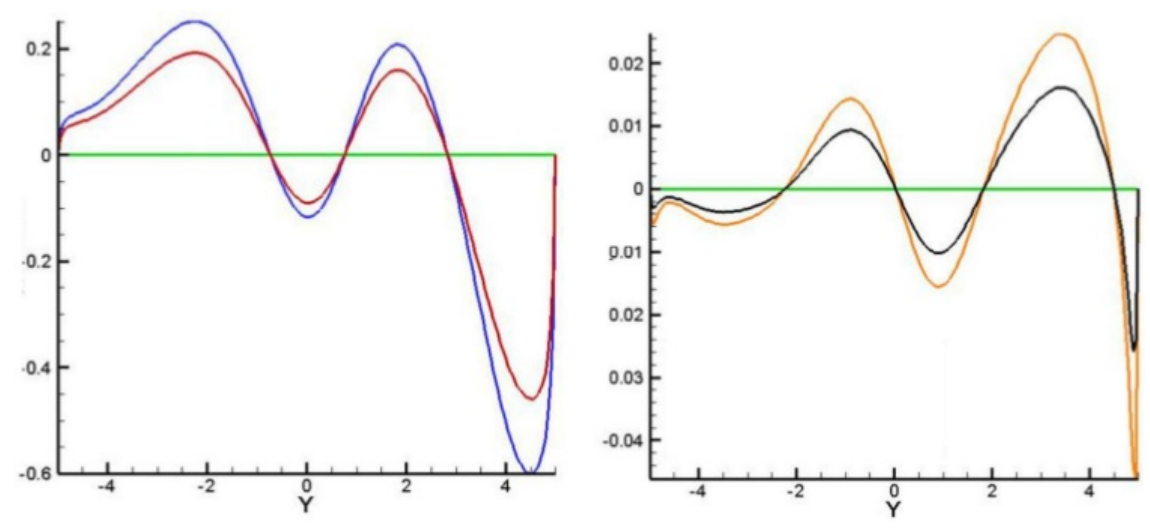

Figura 5.1: Condição de contorno de saída na solução adjunta utilizando o grau de precisão do polinômio interpolador igual a 25. Comparação entre os lados esquerdo e direito da equação 5.1. Esquerda, componente $\varphi_{x}$, onde em vermelho é o lado esquerdo da equação e em azul, o lado direito. Direita, componente $\varphi_{y}$, onde em laranja é o lado esquerdo e em preto o lado direito

Não se sabe a causa deste comportamento da velocidade adjunta, sendo que o teste foi repetido algumas vezes para comprovar o erro. Buscou-se construir uma malha com a mesma geometria do exemplo e alterar o valor de Re, porém o resultado foi o mesmo. A única mudança nos resultados ocorreu quando se alterou o grau do polinômio interpolador, onde a precisão da velocidade adjunta aumentou com o aumento do grau do mesmo. 
As figuras 5.1 e 5.2 apresentam a variação da precisão de $\varphi$ em relação a dois graus diferentes do polinômio interpolador. Todos os resultados vieram da simulação bidimensional mencionada acima, com $R e=30$. A primeira figura corresponde a condição de contorno de saída no caso do grau igual a 25 com as diferenças entre os dois lados da equação 5.1.

Já a figura 5.2 representa a condição de saída adjunta com o grau do polinômio interpolador igual a 50. Neste caso, a precisão da condição de contorno na saída foi semelhante as outras condições de contorno. Assim, pode-se considerar essa solução como a que atende integralmente toda a teoria proposta. Porém o aumento significativo do grau do polinômio interpolador fez o custo computacional tornar-se elevado.
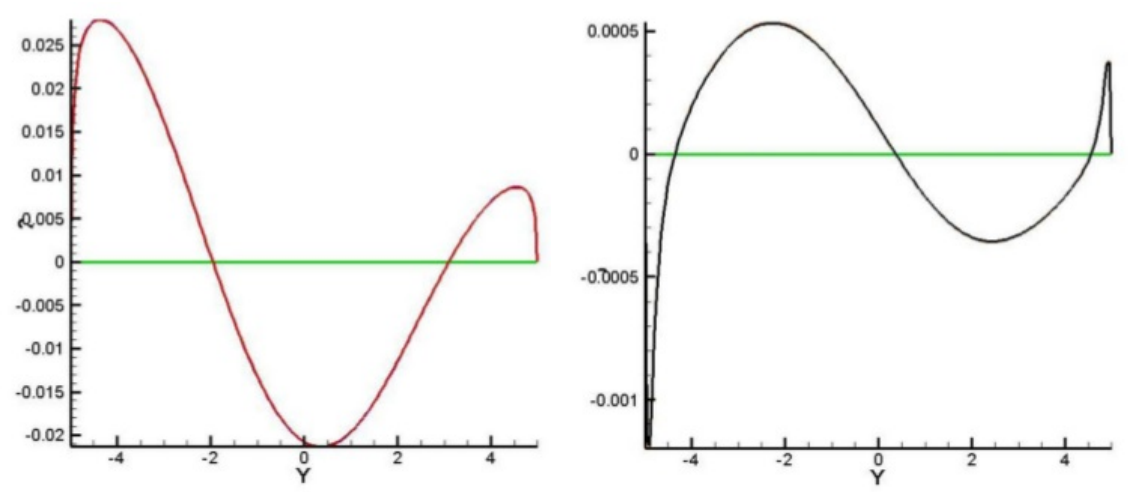

Figura 5.2: Condição de contorno de saída na solução adjunta utilizando o grau de precisão do polinômio interpolador igual a 25. Comparação entre os lados esquerdo e direito da equação 5.1. Esquerda, componente $\varphi_{x}$, onde em vermelho é o lado esquerdo da equação e em azul, o lado direito. Direita, componente $\varphi_{y}$, onde em laranja é o lado esquerdo e em preto o lado direito

A figura 5.3, já mencionada, apresenta as linhas de corrente da velocidade adjunta na solução com melhor precisão. No lado esquerdo, é apresentado o detalhe da solução do escoamento próximo ao cilindro, onde mostra a bolha de separação presa a jusante do cilindro. A solução adjunta em todo o domínio aparece na figura ao lado. Percebe-se na solução adjunta, a entrada do domínio se comporta como uma parede, seguindo exatamente a condição de contorno imposta. Já o cilindro, com as condições de entrada (fonte) e saída (sorvedouro) apresentou o comportamento de um dipolo como esperado. 

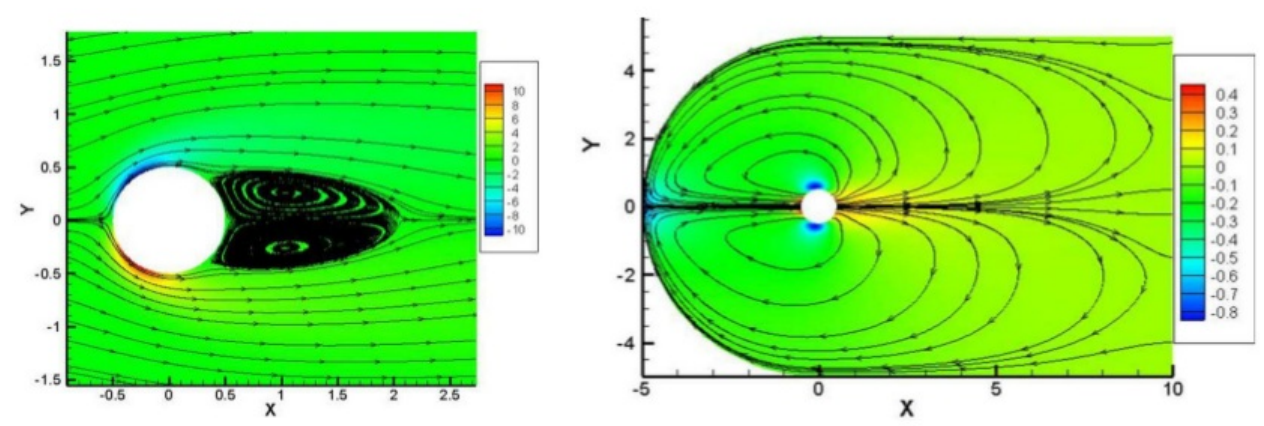

Figura 5.3: Escoamento bidimensional ao redor do cilindro, para $\mathrm{Re}=30 \mathrm{e}$ grau do polinômio interpolador igual a 50. A esquerda, detalhe na região do cilindro. A direita, solução adjunta em todo o domínio computacional 


\section{Conclusões E Trabalhos FUTUROS}

Para o desenvolvimento futuro do método adjunto são necessárias etapas preliminares para se ter um desenvolvimento robusto do método. O presente trabalho conseguiu com sucesso atender a todas estas etapas iniciando com uma escolha acertada do problema a ser estudado pois o splitter plate é um exemplo de problema de interesse na engenharia que possui uma configuração otimizada.

A partir das orientações oriundas das referências bibliográficas, produziu-se uma geometria de malha que reduzisse o custo da simulação numérica, sem afetar a precisão do cálculo. Com o estudo do método numérico baseado nos elementos espectrais, as simulações foram validadas com resultados experimentais.

A análise sistemática que foi realizada conseguiu mapear diversas regiões do universo de soluções, gerando um banco de dados que possui resultados para os 5 parâmetros que são mais estudados neste tipo de problema: A média do coeficiente de arrasto $\left(C_{d}\right)$, o coeficiente de pressão na base do cilindro $\left(C_{p b}\right)$, a amplitude máxima da oscilação do coeficiente de sustentação $\left(C_{l}\right)$, o número de Strouhal $(S t)$ e o comprimento de formação dos vórtices $\left(l_{f}\right)$.

Os resultados de $g / d$ crítico seguiram o que foi encontrado por Kawai (1990) em seus modelos numéricos. Em virtude do valor do número de Reynolds ser baixo, chegou-se a valores de GAP critico que são aproximadamente metade do que foi encontrado nos resultados experimentais de Igarashi (1982). Como foram feitas simulações entre $80 \leq R e \leq 140$, ficou clara a depedência do GAP crítico em relação a $R e$ nessa faixa.

Uma particularidade dos resultados numéricos é o fato de terem conseguido refletir a forma das curvas $g / d$ versus $-C_{p b}$ e $g / d$ versus $S t$ produzidas por Igarashi (1982) em seus resultados experimentais e apresentadas na figura 1.5, com a presença de protuberâncias nas curvas, caracterizando pontos de máximo ou mínimo locais. Este será um teste interessante para o método adjunto pois dependendo do tamanho do passo da variação dos parâmetros de controle e do valor de 
$g / d$ da geometria inicial, ele possivelmente terá dificuldades em chegar ao valor de GAP crítico.

Quanto a formulação do método adjunto, o fato de se conseguir evitar o cálculo das derivadas da medida de mérito em relação aos parâmetros do escoamento reduz drasticamente o custo computacional, pois não necessita de simulações adicionais para o cálculo do gradiente de sensibilidade. Adicionalmente, há uma boa flexiblidade quanto a mudança da função objetivo. Deve-se acrescentar que, em virtude desta flexibilidade, muitos problemas de hidrodinâmica de interesse deste trabalho podem ser solucionados.

Apesar do custo de processamento elevado, os primeiros resultados da solução adjunta mostram que o modelo computacional está próximo da formulação teórica. Obviamente, mais testes são necessários, além de se iniciar as primeiras estimativas do valor do gradiente de sensibilidade, que serão comparados com o gradiente cálculado a partir do método de diferenças finitas.

Sendo assim, os trabalhos futuros deverão concentrar busca da resposta de duas questões importantes:

- O alto grau do polinômio interpolador para se obter uma precisão adequada não era esperado e deve-se investigar o motivo deste custo computacional elevado.

- O modelo da solução adjunta possui uma limitação do perfil de velocidades na condição de saída, sendo possível neste momento calcular apenas com perfis estacionários. O código deve ser explorado para se inserir na formulação o produto (u.n) e assim ampliar a quantidade de casos que podem ser estudados, chegando inclusive nas condições que foram feitas as simulações do método Tentativa e erro no capítulo 3 .

Encerrando este trabalho, pode-se afirmar que os objetivos principais foram cumpridos, garantindo uma base fundamental para o desenvolvimento do método adjunto aplicado as equações de Navier-Stokes como ferramenta importante na pesquisa e aperfeiçoamento dos projetos de engenharia. 


\section{REFERÊNCIAS}

ALONSO, J. J.; KROO, I. M. Advanced algorithms for design and optimization of quiet supersonic platforms. In: AIAA Computational Fluid Dynamics Conference. Reno, NV: [s.n.], 2002. AIAA-2002-0144.

APELT, C. J.; WEST, G. S.; SZEWCZYK, A. A. The effects of wake splitter plates on bluff-body flow in the range $10^{4}<R<5 \times 10^{4}$. Journal of Fluid Mechanics, v. 61, n. 1, p. 187-198, 1973.

ASSI, G. R.; BEARMAN, P. W.; KITNEY, N. Low drag solutions for suppressing vortex-induced vibration of circular cylinders. Journal of Fluids and Structures, v. 25, n. 4, p. 666-675, 2009.

BARKLEY, D.; BLACKBURN, H. M.; SHERWIN, S. J. Direct optimal growth for timesteppers. International Journal for Numerical Methods in Fluids, v. 1, n. 231, p. 1-21, September 2002.

BEARMAN, P. W. Investigation of the flow behind a two-dimensional model with a blunt trailing edge and fitted with splitter plates. Journal of Fluid Mechanics, v. 21, n. 2, p. 241-255, 1965.

BEARMAN, P. W.; CURRIE, I. G. Pressure fluctuation measurements on an oscillating circular cylinder. Journal of Fluid Mechanics, v. 91, n. 4, p. 661-667, 1979 .

BORTHWICK, A. Comparison between two finite-difference schemes for computing the flow around cylinder. International Journal for Numerical Methods of fluids, v. 6, p. n.5 275-290, 1986.

BRAZA M. CHASSAING, P.; MINH, A. Numerical study and physical analysis of pressure and velocity fields in the near wake of circular cylinder. Journal of Fluid Mechanics, v. 165, p. 79-130, 1986.

CABUK, H.; SUNG, C.-H.; MODI, V. Adjoint operator approach to shape design for incompressible flows. In: 3rd International Conference on Inverse Design Concepts and Optimization in Engineering Sciences (ICIDES). College Park, PA: [s.n.], 1991. p. 391-404.

CACUCI, D. G.; WEBER; F., C.; M., O. E.; MARABLE, J. H. Sensitivity theory for general systems of non-linear equations. Nuclear Science and Engineering, v. 75, p. 88-110, 1980.

CARMO, B. S. Estudo Numérico do Escoamento ao Redor de Dois Cilindros Alinhados. Dissertação (M.Sc. Thesis) — Universidade de São Paulo, Brasil, 2005.

CARRIER, G. F.; PEARSON, C. E. Partial Differential Equations. 2nd. ed. NY: Academic Press, 1988. Theory and Technique. 
CEZE, M. A. B. Projeto Inverso Aerodinâmico Utilizando o Método Adjunto Aplicado às Equações de Euler. Dissertação (Mestrado) — Escola Politécnica da Universidade de São Paulo, Agosto 2008.

CHIEREGATTI, B. G. Otimização aerodinâmica de aerofólios utilizando o método adjunto. Trabalho de Graduação — EPUSP. 2008.

DUTA, M. C.; GILES, M. B.; CAMPOBASSO, M. S. The harmonic adjoint approach to unsteady turbomachinery design. Int. J. Numer. Meth. Fluids, v. 40, p. 323-332, 2002.

FENG, C. C. The measurement of vortex-induced effects in a flow past stationary and oscillating circular and D-section cylinders. Dissertação (M.Sc. Thesis) University of British Columbia, Canada, 1968.

FLüGGE, W. Tensor Analysis and Continuum Mechanics. 1st. ed. N.Y.: Springer-Verlag, 1972.

GELFAND, I. M.; FOMIN, S. V. Calculus of Variations. 1st. ed. N.J.: Prentice-Hall, Inc., 1963. (Selected Russian Publications in Mathematical Sciences). Translated and Edited by R. A. Silverman.

GERRARD, J. The mechanics of the formation region of vortices behind bluff bodies. Journal of Fluid Mechanics, v. 25, n. 2, p. 401-413, 1966.

GILES, M.; PIERCE, N.; SüLI, E. Progress in adjoint error correction for integral functionals. Comput Visual Sci, v. 6, p. 113-121, 2004.

GILES, M.; SüLI, E. Adjoint methods for pdes: a posteriori error analysis and postprocessing by duality. Acta Numerica, v. 11, p. 145-236, 2002.

GILES, M. B.; PIERCE, N. A. Adjoint Equations in CFD: Duality, Boundary Conditions and Solution Behavior. 1997. AIAA Paper 97-1850.

GILES, M. B.; PIERCE, N. A. On the properties of solutions of the adjoint Euler equations. In: BAINES, M. J. (Ed.). Numerical Methods for Fluid Dynamics VI. ICFD. [S.l.: s.n.], 1998.

GILES, M. B.; PIERCE, N. A. Superconvergent lift estimates through adjoint error analysis. 1998.

Http:// citeseerx.ist.psu.edu/viewdoc/summary?doi=10.1.1.27.152.

GILES, M. B.; PIERCE, N. A. Adjoint recovery of superconvergent functionals from approximate solutions of partial differential equations. Oxford, August 1999.

GILES, M. B.; PIERCE, N. A. Improved lift and drag estimates using adjoint Euler equations. 1999. AIAA Paper 99-3293.

GILES, M. B.; PIERCE, N. A. An introduction to the adjoint approach to design. Flow, Turbulence and Control, v. 65, n. 3, p. 393-415, March 2000.

GILES, M. B.; PIERCE, N. A. Analytic adjoint solutions for the quasi-1d Euler equations. Journal of Fluid Mechanics, v. 426, p. 327-345, 2001. 
GIORIA, R. S. Estudo da Estabilidade Secundária da Esteira de um Cilindro em Oscilação Forçada. Tese (Doutorado) — Escola Politécnica da Universidade de São Paulo, 2009. Text of the Qualifying Exam.

HALL, M.; CACUCI, D. Physical interpretation of the adjoint functions for sensitivity analysis of atmospheric models. Journal of Atmospheric Sciences, v. 40, p. 2537-2546, October 1983.

HAYASHI, M. T. Estudo Conceitual do Problema Adjunto baseado nas Equações de Euler para Aplicações de Otimização Aerodinâmica. Dissertação (Mestrado)

- Escola Politécnica da Universidade de São Paulo, Fevereiro 2009.

HIRSCH, C. Numerical Computation of Internal and External Flows. 1st. ed. NY: John Wiley \& Sons, 1994. (Wiley Series in Numerical Methods in Engineering, II). Computational Methods for Inviscid and Viscous Flows.

HIRSCH, C. Numerical Computation of Internal and External Flows. 1st. ed. NY: John Wiley \& Sons, 1994. (Wiley Series in Numerical Methods in Engineering, I). Fundamentals of Numerical Discretization.

IGARASHI, T. Investigation on the flow behind a circular cylinder with a wake splitter plate. Bulletin of the JSME, v. 25, n. 202, p. 528-535, 1982.

IGARASHI, T. Correlation between heat transfer and fluctuating pressure in separated region of a circular cylinder. International Journal of Heat and Mass Transfer, v. 27, n. 6, p. 927-937, 1984.

JAMESON, A. Aerodynamic design via control theory. In: 12th IMACS World Congress on Scientific Computation. Paris: [s.n.], 1988. (MAE Report 1824).

JAMESON, A. Aerodynamic design via control theory. Journal of Scientific Computing, v. 3, p. 233-260, 1988.

JAMESON, A. Optimum aerodynamic design using cfd and control theory. In: AIAA 95-1729-CP. [S.l.: s.n.], 1995.

JAMESON, A.; KIM, S. Reduction of the adjoint gradient formula for aerodynamic shape optimization problems. AIAA Journal, v. 41, n. 11, p. 2114-2129, November 2003.

JAMESON, A.; KIM, S. Reduction of the adjoint gradient formula in the continuous limit. In: AIAA Computational Fluid Dynamics Conference. [S.l.: s.n.], 2003. AIAA 2003-0040.

JAMESON, A.; MARTINELLI, L.; PIERCE, N. A. Optimum aerodynamic design using the Navier-Stokes equations. In: AMERICAN INSTITUTE OF AERONAUTICS AND ASTRONAUTICS. 35th Aerospace Sciences Meeting \& Exhibit. Reno, NV: AIAA, 1997. p. 1-20.

JAMESON, A.; MARTINELLI, L.; PIERCE, N. A. Optimum aerodynamic design using the Navier-Stokes equations. Theoretical and Computational Fluid Dynamics, v. 1, n. 10, p. 213-237, 1998.

JAMESON, A.; SRIRAM, A.; MARTINELLI, L. A continuous adjoint method for unstructured grids. In: AIAA Computational Fluid Dynamics Conference. Orlando, FL: [s.n.], 2003. AIAA 2003-3955. 
KAPLAN, W. Advanced Calculus. N.Y.: Addison-Wesley Pub. Co., 1971.

KARMAN, T. von. Uber den mechanismus des widerstandes den ein beweteger korper in einer flussigkeit erfarht. Gottinger Nachrichten Math-Phys, p. 509-517, 1911.

KARNIADAKIS, G.; ISRAELI, M.; ORSZAG, S. High-order splitting methods for incompressible navier-stokes equations. Journal of Computational Physics, v. 97, p. 414-443, 1991.

KARNIADAKIS G.E.; SHERWIN, S. Spectral element methods for CFD. New York: Oxford University Press, 1999.

KAWAI, H. Discrete vortex simulation for flow around a circular cylinder with a splitter plate. Journal of Wind Engineering and Industrial Aerodynamics, v. 33, n. 1,1990 .

KIM, H.; NAKAHASHI, K. Unstructured adjoint method for Navier-Stokes equations. JSME International Journal, v. 48, n. 2, 2005.

KIM, H. J.; SASAKI, D.; OBAYASHI, S.; NAKAHASHI, K. Aerodynamic optimization of supersonic transport wing using unstructured adjoint method. AIAA Journal, v. 39, n. 6, p. 1011-1020, June 2001.

KIM, J. J. A. S.; JAMESON, A. A gradient accuracy study for the adjoint-based Navier-Stokes design method. In: AIAA Computational Fluid Dynamics Conference. [S.l.: s.n.], 1999. AIAA-99-0299.

KIM, S. Design Optimization of High-Lift Configurations Using a Viscous Adjoint-Based Method. Tese (Doutorado) — Stanford University, 2001.

KIM, S.; ALONSO, J. J.; JAMESON, A. Multi-element high-lift configuration design optimization using viscous continuous adjoint method. Journal of Aircraft, v. 41, n. 5, September-October 2004.

KURUVILA, G.; TAASAN, S.; SALAS, M. D. Airfoil Optimization by the One-Shot Method, Optimum Design Methods in Aerodynamics. 1994. AGARD-FDP-VKI Special Course.

KWON, K.; CHOI, H. Control of laminar vortex shedding behind a circular cylinder using splitter plates. Physics of Fluids, v. 8, n. 2, p. 479-486, 1996.

LECOINTE, Y.; PIQUET, J.; PLANTEC, J. Flow structure in the wake of an oscillating cylinder. Forum on unsteady separation, p. 147-157, 1987.

LUSTERNICK, L. A.; SOBOLEV, V. J. Elements of Functional Analysis. 1st. ed. Delhi: Hindustan Pub. Co., 1961. (Russian Monographs and Texts on Advanced Mathematics and Physics, V).

MANI, K.; MAVRIPLIS, D. Unsteady discrete adjoint formulation for two-dimensional flow problems with deforming meshes. AIAA Journal, v. 46, n. 6, p. 1351-1364, June 2008.

MENEGHINI, J. R. Numerical Simulation of Bluff Body Flow Control Using a Discrete Vortex Method. Tese (Doutorado) - Imperial College of Science, Technology and Medicine, University of London, London, UK, 1993. 
MENEGHINI, J. R. Numerical simulation of flow interference between two circular cylinders in tandem and side-by-side arrangements. Journal of Fluid and Estructures, v. 15, n. 1, p. 327-350, 2001.

MENEGHINI, J. R. Geração e Desprendimento de Vórtices no Escoamento ao Redor de Cilindros. Tese (Tese de Livre Docência), São Paulo, 2002.

MENEGHINI, J. R.; BEARMAN, P. W. Numerical simulation of high amplitude oscillatory flow about a circular cylinder. Journal of Fluids and structures, v. 9, p. 435-455, 1995.

MOHAMMADI, B.; PIRRONEAU, O. Applied Shape Optimization for Fluids. 1st. ed. [S.1.]: Oxford University Press, 2001.

MORSE, P. M.; FESHBACH, H. Methods of Theoretical Physics. 1st. ed. NY: MacGraw-Hill, 1953.

MORSE, P. M.; FESHBACH, H. Methods of Theoretical Physics. 1st. ed. NY: MacGraw-Hill, 1953.

NADARAJAH, S.; JAMESON, A. Optimum shape design for unsteady three-dimensional viscous flows using a non-linear frequency domain method. In: AMERICAN INSTITUTE OF AERONAUTICS AND ASTRONAUTICS. 24th Applied Aerodynamics Conference. San Francisco, CA: AIAA, 2006.

NADARAJAH, S. K. The Discrete Adjoint Approach to Aerodynamic Shape Optimization. Tese (Doutorado) — Stanford University, 2003.

NADARAJAH, S. K.; JAMESON, A. A comparison of the continuous and discrete adjoint approach to automatic aerodynamic optimization. In: AIAA 38th Aerospace Sciences Meeting and Exhibit. Reno, NV: [s.n.], 2000. AIAA 2000-0667.

NADARAJAH, S. K.; JAMESON, A. Optimum shape design for unsteady flows with time-accurate continuous and discrete adjoint methods. AIAA Journal, v. 45, n. 7, p. 1478-1491, July 2007.

PIERCE, N.; GILES, M. Adjoint and defect error bounding and correction for functional estimates. Journal of Computational Physics, v. 200, p. 769-794, 2004 .

PIRRONEAU, O. On optimal profiles in stokes flow. Journal of Fluid Mechanics, v. 59, n. 1, p. 117-128, 1973.

PIRRONEAU, O. On optimal design in fluid dynamics. Journal of Fluid Mechanics, v. 64, n. 1, p. 97-110, 1974.

PIRRONEAU, O. Optimal Shape Design for Elliptic Systems. 1st. ed. [S.1.]: Springer-Verlag, 1983.

PISKUNOV, N. S. Differential and Integral Calculus. 1st. ed. Moscow: Mir Publishers, 1968. Translated from Russian by G. Yankovsy.

QIAO, Z.; QIN, X.; YANG, X. Wing design by solving adjoint equations. In: AMERICAN INSTITUTE OF AERONAUTICS AND ASTRONAUTICS. 40th Aerospace Sciences Meeting \& Exhibit. Reno, NV: AIAA, 2002. 
RAYLEIGH, J. W. S. The Theory of sounde. [S.l.: s.n.], 1896.

ROSHKO, A. On the development of turbulent wakes from vortex streets. [S.1.], 1954.

ROSHKO, A. On the drag and sheddind frequency of two-dimensional bluff bodies. [S.l.], 1954.

SANTOS, L. C. C. A Study on Aerodynamic Design Optimization Using An Adjoint Method. Braunschweig, July 1995.

SERSON, D.; MENEGHINI, J. R. Simulações Numéricas do Escoamento ao redor de um Cilindro com Splitter Plate. Brazil, 2010. Projeto FAPESP $2009 / 14233-1$.

SHUKLA, S.; GOVARDHAN, R. N.; ARAKERI, J. H. Flow over a cylinder with a hinged-splitter plate. Journal of Fluids and Structures, v. 25, n. 4, p. 713-720, 2009.

TAASAN, S.; KURUVILA, G.; SALAS, M. D. Aerodynamic design and optimization in one shot. In: 30th Aerospace Sciences Meeting and Exhibit. Reno, NV: [s.n.], 1992. AIAA 92-0025.

T.H., M. B. Y. D. O. Fundamentos da Mec $\widetilde{A} \notin n i c a$ dos FluÃdos. SÃ£o Paulo: Editora Edgard $\mathrm{Bl} \tilde{\mathrm{A}} \frac{1}{4}$ cher, 2004.

THOMAS, J. P.; HALL, K. C.; DOWELL, E. H. Discrete adjoint approach for modeling unsteady aerodynamic design sensitivities. AIAA Journal, v. 43, n. 9, p. 1931-1936, September 2005.

UNAL, F. M.; ROCKWELL, D. On vortex formation from a cylinder. Part 1. The initial instability. Journal of Fluid Mechanics, v. 190, p. 491-512, 1988.

UNAL, F. M.; ROCKWELL, D. On vortex formation from a cylinder. Part 2. Control by splitter-plate interference. Journal of Fluid Mechanics, v. 190, p. 513-529, 1988.

VENDITTI, D. A.; DARMOFAL, D. L. A multilevel error estimation and grid adaptive strategy for improving the accuracy of integral outputs. 1999. AIAA Paper 99-3292.

VENDITTI, D. A.; DARMOFAL, D. L. Adjoint error estimation and grid adaptation for functional outputs: Application to quasi-one-dimensional flow. Journal of Computational Physics, v. 164, p. 204-227, 2000.

VENDITTI, D. A.; DARMOFAL, D. L. Anisotropic grid adaptation for functional outputs: application to two-dimensional viscous flows. Journal of Computational Physics, v. 187, p. 22-46, 2003.

VOLPE, E.; CHIEREGATTI, B.; LIMA, J.; MENEGHINI, J. Continuous Formulation of the'Adjoint Problem for Unsteady Imcompressible Navier Stokes Flows. SP, June 2012.

VOLPE, E. V. Estudo da Transferência de Calor e Quantidade de Movimento no Escoamento Laminar através de Passagens com Geometria Arbitrária: Modelos Compressivel e Incompressível. Dissertação (Mestrado) - Escola Politécnica da Universidade de São Paulo, 1993. 
VOLPE, E. V. A11 - Inverse Aerodynamic Design Module. SP, August 2004. Research Project: Advanced Applications of Computational Fluid Dynamics to High Performance Aircraft.

VOLPE, E. V. A3 - Inverse Aerodynamic Design Applications. SP, August 2006. Research Project: Advanced Applications of Computational Fluid Dynamics to High Performance Aircraft.

VOLPE, E. V.; SANTOS, L. C. C. Boundary and internal conditions for adjoint fluid flow problems - applications to quasi-1D Euler equations. Journal of Engineering Mathematics, v. 65, n. 1, p. 1-24, September 2009.

WILLIAMSON, C. 2-d and 3-d aspects of the wake of a cylinder, and their relation to wake computations. In: ANDERSON, C.;GREENGARD, C. (Ed.) Vortex Dynamics and Vortex Method, p. 719-751, 1991. 


\section{ApÊNDICE A - Forma GeneralizAdA DAS EquAÇÕes DE NAVIER STOKES}

Este anexo tem como objetivo de apresentar toda formulação das equações de Navier Stokes na forma generalizada. Na literatura, as equações aparecem em duas formas: Forma extendida, onde a operadores de diferenciação são todos na forma generalizada e a forma reduzida, que abrange tanto as coordenadas cartesianas e as transformadas. O primeiro caso utiliza o formalismo do tensor generalizado (FLüGGE, 1972; VOLPE, 1993), já o segundo caso, as equações são obtidas com uma transformada de coordenadas (JAMESON; MARTINELLI; PIERCE, 1998).

Neste trabalho, foram utilizadas as duas formas, sendo que será provado que elas são consideradas equivalentes. Previamente deve-se conhecer os tensores generalizados, onde recomenda-se a leitura de (FLüGGE, 1972) como referência no assunto. No descritivo a seguir, utilizou-se a mesma notação apresentada na referência:

\section{A.1 Introdução}

Inicialmente assume-se que o domínio do escoamento possa ser representado pelo sistema cartesiano e que o espaço transformado é descrito em coordenadas generalizadas. A figura A.1 abaixo apresenta uma representação esquemática:

$$
\left\{\begin{array}{l}
\text { Domínio do Escoamento } \Rightarrow\left(x^{j^{\prime}}, t\right) \\
\text { Espaço Tranformado } \Rightarrow\left(\xi^{i}, t\right)
\end{array}\right.
$$

Para distinguir os dois sistemas, os elementos dos tensores serão designados com apóstrofes, quando se tratar do sistema cartesiano e sem apóstrofe para o espaço transformado. Os operadores de transformação são definidos como sendo: 


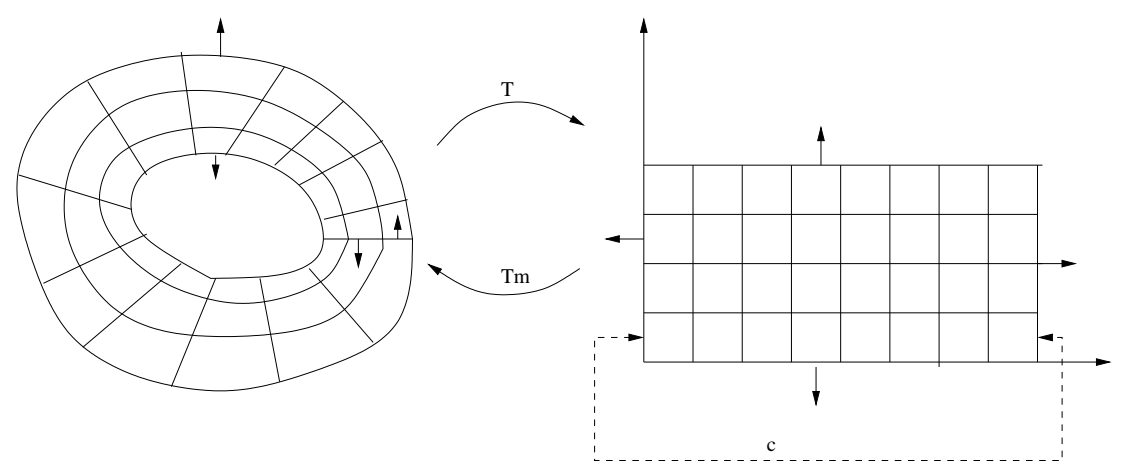

Figura A.1: Espaços Físico e Tranformado. Operador de tranformada, $\beta$ e sua matriz inversa $\beta^{-1}$

$$
\left\{\begin{array}{ccc}
\beta \Rightarrow \beta_{j^{\prime}}^{i}=\frac{\partial \xi^{i}}{\partial x^{j^{\prime}}} \quad: \quad u^{i}=\beta_{j^{\prime}}^{i} u^{j^{\prime}} \\
\beta^{-1} \Rightarrow \beta_{i}^{j^{\prime}}=\frac{\partial x^{j^{\prime}}}{\partial \xi^{i}} \quad: \quad u^{j^{\prime}}=\beta_{i}^{j^{\prime}} u^{i}
\end{array}\right.
$$

e a Jacobiana de Transformação é dada por $J=\operatorname{det}\left(\beta_{i}^{j^{\prime}}\right)$. É importante ressaltar que as componentes dos vetores covariantes e contravariantes $\left(u^{i}\right.$ e $u_{i}$, respectivamente) são coincidentes somente no sistema cartesiano.

A notação das equações de Navier Stokes em sua forma completa pode ser escrita da seguinte maneira (HIRSCH, 1994b):

$$
\left\{\begin{array}{l}
\frac{\partial \rho}{\partial t}+\nabla \cdot(\rho \mathbf{u})=0 \\
\frac{\partial(\rho \mathbf{u})}{\partial t}+\nabla \cdot(\rho \mathbf{u u})=-\nabla p+\nabla \cdot \tau \\
\frac{\partial\left(\rho e_{o}\right)}{\partial t}+\nabla \cdot\left(\rho \mathbf{u} h_{o}\right)=\nabla \cdot(k \nabla T)+\nabla \cdot(\tau \cdot \mathbf{u})
\end{array}\right.
$$

Os parâmetros $e_{o}$ and $h_{o}$ são designados como a energia e a entalpia de estagnação respectivamente:

$$
e_{o}=e_{i}+\frac{u^{2}}{2} ; \quad h_{o}=h+\frac{u^{2}}{2}
$$

onde $e_{i}$ e $h$ representam a energia térmica e a entalpia, respectivamente e $u \equiv|\mathbf{u}|$. O tensor das tensões viscosa $\tau$ é dado por:

$$
\boldsymbol{\tau}=\mu\left[\left(\nabla \mathbf{u}+\nabla \mathbf{u}^{T}\right)-\frac{2}{3} \mathbf{I} \nabla \cdot \mathbf{u}\right]
$$

onde o símbolo I se mantém como a matriz identidade. Ao longo deste anexo, as propriedades de transporte $\mu$ e $k$ serão assumidas como constantes.

A versão completa das equações A.3 é obtida a partir dos operadores diferen- 
ciais em sua forma generalizada (FLüGGE, 1972). Esta operação implica em:

$$
\left\{\begin{array}{l}
\partial_{o} \rho+\left.\left(\rho u^{k}\right)\right|_{k}=0 \\
\partial_{o}\left(\rho u^{i}\right)+\left.\left(\rho u^{i} u^{j}\right)\right|_{j}=-\left.\left(g^{i j} p\right)\right|_{j}+\left.\tau^{i j}\right|_{j} \\
\partial_{o}\left(\rho e_{o}\right)+\left.\left(\rho h_{o} u^{i}\right)\right|_{i}=\left.\left(\left.g^{i j} k T\right|_{j}\right)\right|_{i}+\left.\left(\tau^{i j} u_{j}\right)\right|_{i}
\end{array}\right.
$$

onde o símbolo $\partial_{o}$ representa a derivada no tempo $\partial_{o}=\partial / \partial t$. Na mesma notação, o tensor das tensões generalizado é dado por:

$$
\tau^{i j}=\mu\left[\left(\left.u^{i}\right|^{j}+\left.u^{j}\right|^{i}\right)-\left.\frac{2}{3} g^{i j} u^{k}\right|_{k}\right]
$$

A princípio, há duas abordagens para se obter a versão reduzida das equações acima. A primeira pressupõe a aplicação do operador de transformação (Eq. A.2) aplicado nas equações de Navier Stokes no plano cartesiano; ou a segunda alternativa que parte da forma generalizada completa (Eq. A.6) e com um trabalho de álgebra obtém-se a forma reduzida.

Na literatura, a primeira abordagem é preferida em relação a segunda devido a sua simplicidade (JAMESON; MARTINELLI; PIERCE, 1998). Entretanto, neste trabalho será utilizado a segunda alternativa, com o propósito de apresentar a total equivalência entre as duas abordagens.

\section{A.2 A Álgebra Tensorial Generalizada}

Como mencionado acima, algumas operações serão consideradas como entendidas pelo leitor a partir de seu conhecimento prévio. Esta seção irá focar nos conceitos que afetam a dedução do problema, com alguns resultados sendo provados matematicamente.

A dedução iniciará a partir dos operadores diferenciais das equações (A.6)(A.7). A derivada covariante define a forma generalizada do operador gradiente $(\nabla)($ FLüGGE, 1972). Para os escalares, a derivada simplesmente se reduz a derivada parcial $\left.p\right|_{k}=p_{, k}$, onde a vírgula indica a diferenciação parcial, ( $)_{, k}=\partial / \partial \xi^{k}$.

Entretanto, os vetores serão diferentes:

$$
\left\{\begin{array}{l}
\left.u^{i}\right|_{j}=u_{, j}^{i}+u^{k} \Gamma_{j k}^{i} \\
\left.u_{i}\right|_{j}=u_{i, j}-u_{k} \Gamma_{i j}^{k}
\end{array}\right.
$$

Os resultados são tensores de segunda ordem, e suas variações podem ser trocadas com o tensor métrico $g^{i j}:\left.u_{i}\right|_{j} g^{i k} g^{j p}=\left.u^{k}\right|^{p}$. Para os tensores de segunda ordem, 
temos que (FLüGGE, 1972):

$$
\left\{\begin{array}{l}
\left.A_{i j}\right|_{k}=A_{i j, k}-A_{l j} \Gamma_{i k}^{l}-A_{i l} \Gamma_{k j}^{l} \\
\left.A^{i}\right|_{k}=A_{j, k}^{i}+A_{j}^{l} \Gamma_{k l}^{i}-A^{i} \Gamma_{j k}^{l} \\
\left.A_{i}^{j}\right|_{k}=A_{i, k}^{j}-A_{l}^{j} \Gamma_{i k}^{l}+A_{i}^{l} \Gamma_{k l}^{j} \\
\left.A^{i j}\right|_{k}=A_{, k}^{i j}+A^{l j} \Gamma_{k l}^{i}+A^{i l} \Gamma_{k l}^{j}
\end{array}\right.
$$

e o resultado gera tensores de terceira ordem, onde suas variações também podem ser trocadas com o tensor métrico.

É importante ressaltar duas propriedades relevantes das derivadas covariantes: Ela satisfaz a regra de diferenciação do produto de tensores e a ordem da segunda derivada covariante pode ser trocada contando que o vetor de espaço não tenha curvatura intríseca, mesmo que possa ser representado por coordenadas curvilíneas (FLüGGE, 1972).

$$
\begin{aligned}
\left.\left(u_{i} v_{j}\right)\right|_{k} & =\left.u_{i}\right|_{k} v_{j}+\left.u_{i} v_{j}\right|_{k} \\
\left.\left(u_{i} A^{i j}\right)\right|_{j} & =\left.u_{i}\right|_{j} A^{i j}+\left.u_{i} A^{i j}\right|_{j} \\
\left.\left(u^{i} A_{i j}\right)\right|^{j} & =\left.u^{i}\right|^{j} A_{i j}+\left.u^{i} A_{i j}\right|^{j} \\
\left.\left.u_{i}\right|_{j}\right|_{k} & =\left.\left.u_{i}\right|_{k}\right|_{j} \quad \text { em espaços com nenhuma curvatura intríseca }
\end{aligned}
$$

Em todas as aplicações consideradas neste anexo, espaço vetorial, $\mathbf{R}^{3}$, não possui curvatura intríseca e pode ser descrito tanto com coordenadas cartesianas como com curvilíneas.

O parâmetro $\Gamma_{j k}^{i}$ nas equações acima denota os símbolos de Christoffel. Em suma, eles representam os efeitos da curvatura local do sistema de coordenadas na diferenciação (FLüGGE, 1972). Duas de suas inúmeras propriedades são mais relevantes nas aplicações deste trabalho: Há simetria entre o primeiro e segundo índices, assim, os mesmos podem ser invertidos e o terceiro índice pode ser colocado tanto na parte superior quanto inferior com o auxílio do tensor métrico.

$$
\left\{\begin{array}{l}
\Gamma_{i j}^{k}=\Gamma_{j i}^{k} \\
\Gamma_{i j}^{k} g_{k n}=\Gamma_{i j n}
\end{array}\right.
$$

É importante ressaltar novamente que o resultado acima não é um tensor de terceira ordem. Uma maneira de se calcular os termos de Christoffel em termos dos operadores de transformação será desenvolvida a seguir.

Os símbolos $g_{i j}$ e $g^{i j}$ designam os componentes covariantes e contravariantes no tensor métrico, respectivamente: $(\mathrm{d} s)^{2}=g^{i j} \mathrm{~d} \xi_{i} \mathrm{~d} \xi_{j}=g_{i j} \mathrm{~d} \xi^{i} \mathrm{~d} \xi^{j}$. 
Algumas de suas propriedades também possuem relevância neste trabalho: Os tensores métricos são simétricos e assumem forma diagonal quando associado com um sistema localmente ortogonal de coordenadas.

Em todos os casos considerados, o sistema de coordenadas são ortogonais. Assim, todos os elementos não diagonais do tensor métrico serão sempre zero. Uma identidade útil que permite inverter o tensor métrico, $g^{i j} g_{j k}=\delta_{k}^{i}$, é apresentada abaixo:

$$
\begin{aligned}
g^{i k} & =\delta_{j}^{i} g^{j k}=\frac{1}{2} \epsilon^{i p q} \epsilon_{j p q} g^{j k} \\
& =\frac{1}{2} \epsilon^{i p q} \epsilon^{r l m} g_{r j} g_{p l} g_{q m} g^{j k} \\
& =\frac{1}{2} \epsilon^{i p q} \epsilon^{k l m} g_{p l} g_{q m}
\end{aligned}
$$

onde $\epsilon^{i j k}$ representa o tensor de permutação e $\delta_{j}^{i}$ representa o delta de Kronecker.

Os tensores de permutação, como visto na equação (A.11), estão associados com o espaço transformado e são definidos em termos dos símbolos de permutação como $\epsilon_{i j k}=J e_{i j k}$ para os componentes covariantes e $\epsilon^{i j k}=J^{-1} e^{i j k}$ para os contravariantes. Estes símbolos são definidos como:

$e^{i j k}=e_{i j k}=\left\{\begin{array}{l}+1 \text { if }(i, j, k)=(1,2,3) \text { ou qualquer permutação par da sequência } \\ -1 \text { if }(i, j, k) \text { é qualquer permutação ímpar } \\ 0 \quad \text { if se os dois subíndices são iguais. }\end{array}\right.$

Diferente dos tensores, os símbolos de permutação não são associados com nenhum sistema de coordenadas. Adicionalmente, vale ressaltar que apenas no sistema cartesiano os tensores de permutação coincidem com os símbolos de permutação.

Similar à equação (A.11), o operador da transformada inversa $\left(\beta_{j^{\prime}}^{i} \beta_{k}^{j^{\prime}}=\delta_{k}^{i}\right)$, é obtido a partir de:

$$
\begin{aligned}
\delta_{n}^{k} & =\frac{1}{2} \epsilon^{i j k} \epsilon_{i j n}=\frac{1}{2} \epsilon^{i j k} \epsilon_{r^{\prime} l^{\prime} s^{\prime}} \beta_{i}^{r^{\prime}} \beta_{j}^{l^{\prime}} \beta_{n}^{s^{\prime}} \\
\beta_{p^{\prime}}^{k} \beta_{n}^{p^{\prime}} & =\frac{1}{2 J} e^{i j k} e_{r^{\prime} l^{\prime} s^{\prime}} \beta_{i}^{r^{\prime}} \beta_{j}^{l^{\prime}} \beta_{n}^{s^{\prime}} \\
\beta_{q^{\prime}}^{k} & =\frac{1}{2 J} e^{i j k} e_{r^{\prime} l^{\prime} q^{\prime}} \beta_{i}^{r^{\prime}} \beta_{j}^{l^{\prime}}
\end{aligned}
$$


Nestas equações foram utilizadas as relações entre o tensor de permutação e os símbolos, além da igualdade entre os mesmos no sistema cartesiano (FLüGGE, 1972; VOLPE, 1993).

Multiplicando a última linha da equação acima por J, além de substituir $\left(\beta_{i}^{r^{\prime}} \beta_{j}^{l^{\prime}}\right)$ por suas definições (Eq. (A.2)) e derivar o resultado, obtém-se:

$$
\begin{aligned}
\frac{\partial}{\partial \xi^{k}}\left(J \beta_{q^{\prime}}^{k}\right) & =\frac{1}{2} e^{i j k} e_{r^{\prime} l^{\prime} q^{\prime}}\left(\frac{\partial^{2} x^{r^{\prime}}}{\partial \xi^{k} \partial \xi^{i}} \frac{\partial x^{l^{\prime}}}{\partial \xi^{j}}+\frac{\partial x^{r^{\prime}}}{\partial \xi^{i}} \frac{\partial^{2} x^{l^{\prime}}}{\partial \xi^{k} \partial \xi^{j}}\right) \\
\left(J \beta_{q^{\prime}}^{k}\right)_{, k} & =0
\end{aligned}
$$

onde o valor 0 vem da dupla contração entre o tensor simétrico o símbolo de permutação anti-simétrica $e^{i j k}$. Os resultados acima (Eqs. (A.13) e (A.14)) são de grande relevância para a redução do gradiente adjunto (JAMESON; KIM, 2003a; JAMESON; KIM, 2003b).

Além disso, o uso dos símbolos de permutação com os tensores correspondentes pode gerar uma nova forma para a equação (A.11), mais vantajosa para as aplicações:

$$
g^{i k}=\frac{e^{i p q} e^{k l m}}{2 J^{2}} g_{p l} g_{q m}
$$

onde se reconhece um resultado difundido na literatura (FLüGGE, 1972) que $\operatorname{det}\left(g_{i j}\right) \equiv g=J^{2}$. É importante ressaltar que as equações (A.13) e (A.15) são basicamente a fórmula clássica de matriz inversa:

$$
\mathbf{A}^{-1}=\frac{1}{\operatorname{det}(\mathbf{A})} \mathbf{C}^{T}
$$

Sendo $\mathbf{C}^{T}$ a matriz transposta dos cofatores de $\mathbf{A}$.

Uma expressão para calcular os símbolos de Christofell em temos do operador transformada pode ser obtida a partir da relação com o tensor métrico (FLüGGE, 1972). Substituindo os últimos termos por suas definições e expandindo o resultado, obtém-se que: 


$$
\begin{aligned}
2 \Gamma_{i j k} & =g_{j k, i}+g_{k i, j}-g_{i j, k} \\
& =\frac{\partial}{\partial \xi^{i}}\left(\frac{\partial x^{p^{\prime}}}{\partial \xi^{j}} \frac{\partial x^{p^{\prime}}}{\partial \xi^{k}}\right)+\frac{\partial}{\partial \xi^{j}}\left(\frac{\partial x^{s^{\prime}}}{\partial \xi^{k}} \frac{\partial x^{s^{\prime}}}{\partial \xi^{i}}\right)-\frac{\partial}{\partial \xi^{k}}\left(\frac{\partial x^{q^{\prime}}}{\partial \xi^{i}} \frac{\partial x^{q^{\prime}}}{\partial \xi^{j}}\right) \\
2 \Gamma_{i j k} & =2 \frac{\partial^{2} x^{p^{\prime}}}{\partial \xi^{i} \xi^{j}} \frac{\partial x^{p^{\prime}}}{\partial \xi^{k}} \\
\Gamma_{i j k} & =\left(\beta_{j}^{p^{\prime}}\right)_{, i} \beta_{k}^{p^{\prime}}
\end{aligned}
$$

produzindo o símbolo de Christofell com o terceiro índice inferior.

Duas expressões similares fornecem o símbolo com o terceiro índice levantado. A primeira é obtida a partida da eq. (A.17), combinada com as equações (A.11) e (A.13). O caminho para a derivação é apresentado abaixo:

$$
\begin{aligned}
\Gamma_{k j}^{p} & =\Gamma_{k j l} g^{l p}=g^{l p} \frac{\partial^{2} x^{q^{\prime}}}{\partial \xi^{k} \xi^{j}} \frac{\partial x^{q^{\prime}}}{\partial \xi^{l}} \\
& =\frac{\epsilon^{p s r} \epsilon^{l m n}}{2} g_{s m} g_{r n} \frac{\partial^{2} x^{q^{\prime}}}{\partial \xi^{k} \xi^{j}} \frac{\partial x^{q^{\prime}}}{\partial \xi^{l}} \\
& =\frac{\epsilon^{p s r} \epsilon^{l m n}}{2} \frac{\partial x^{i^{\prime}}}{\partial \xi^{s}} \frac{\partial x^{i^{\prime}}}{\partial \xi^{m}} \frac{\partial x^{v^{\prime}}}{\partial \xi^{r}} \frac{\partial x^{v^{\prime}}}{\partial \xi^{n}} \frac{\partial x^{q^{\prime}}}{\partial \xi^{l}} \frac{\partial^{2} x^{q^{\prime}}}{\partial \xi^{k} \xi^{j}} \\
& =\frac{\epsilon^{p s r} \epsilon^{q^{\prime} i^{\prime} v^{\prime}}}{2} \frac{\partial x^{i^{\prime}}}{\partial \xi^{s}} \frac{\partial x^{v^{\prime}}}{\partial \xi^{r}} \frac{\partial^{2} x^{q^{\prime}}}{\partial \xi^{k} \xi^{j}} \\
& =\frac{e^{p s r} e_{q^{\prime} i^{\prime} v^{\prime}}}{2 J} \frac{\partial x^{i^{\prime}}}{\partial \xi^{s}} \frac{\partial x^{v^{\prime}}}{\partial \xi^{r}} \frac{\partial^{2} x^{q^{\prime}}}{\partial \xi^{k} \xi^{j}} \\
\Gamma_{k j}^{p} & =\beta_{q^{\prime}}^{p}\left(\beta_{j}^{q^{\prime}}\right)_{, k}
\end{aligned}
$$

Na equação acima foi utilizada a igualdade entre os tensores e símbolos de permutação e o símbolos nas coordenadas cartesianas, além da coincidência entre os componentes covariantes e contravariantes, presentes neste caso em particular. Finalmente, utilizando a equação (A.18), obtém-se a expressão final destas aplicações:

$$
\begin{aligned}
\left(\delta_{j}^{p}\right)_{, k}=\left(\beta_{q^{\prime}}^{p} \beta_{j}^{q^{\prime}}\right)_{, k} & =0 \\
\left(\beta_{q^{\prime}}^{p}\right)_{, k} \beta_{j}^{q^{\prime}}+\Gamma_{k j}^{p} & =0 \\
\Gamma_{k j}^{p} & =-\left(\beta_{q^{\prime}}^{p}\right)_{, k} \beta_{j}^{q^{\prime}}
\end{aligned}
$$

Um resultado extremamente importante é a contração entre dois índices dos símbolos de Christoffel. Para derivar esta equação, partimos da relação entre as 
entidades e o tensor métrico que aparecem na primeira linha da eq. (A.17):

$$
\begin{aligned}
& 2 \Gamma_{i j}^{l}=2 \Gamma_{i j k} g^{l k}=g^{l k}\left(g_{j k, i}+g_{k i, j}-g_{i j, k}\right) \\
& 2 \Gamma_{j i}^{i}=g^{i k}\left(g_{j k, i}+g_{k i, j}-g_{i j, k}\right) \\
& 2 \Gamma_{j i}^{i}=g^{i k} g_{j k, i}+g^{i k} g_{k i, j}-g^{i k} g_{j k, i} \\
& 2 \Gamma_{j i}^{i}=g^{i k} g_{k i, j}
\end{aligned}
$$

Passando da segunda para a terceira linha, o produto foi expandido e os índices contraídos do terceiro termo do lado direito foram permutados, assim foi revelada a similaridade com o primeiro termo. Cancelando os mesmos, chega-se ao resultado final, e neste ponto, a equação A.15 pode ser substituída por $g^{i k}$ da equação A.20, chegando a:

$$
2 \Gamma_{j i}^{i}=\frac{1}{J^{2}}\left(\frac{1}{2} e^{i p q} e^{k l m} g_{p l} g_{q m} g_{k i, j}\right)
$$

Alguns termos foram colocados entre parêntesis para ilustrar que os mesmos podem ser comparados com a derivada parcial do determinante de $g_{i j}$ : $g=\operatorname{det}\left(g_{i j}\right)=J^{2}$. O símbolo g será usado apenas por conveniência algébrica. Este determinante pode ser calculado pela seguinte equação (FLüGGE, 1972):

$$
g=\frac{1}{6} e^{i p q} e^{k l m} g_{i k} g_{p l} g_{q m}
$$

Então, obtém-se que:

$$
\begin{aligned}
g_{, j} & =\frac{1}{6} e^{i p q} e^{k l m}\left(g_{i k} g_{p l} g_{q m}\right)_{, j} \\
& =\frac{1}{6} e^{i p q} e^{k l m}\left(g_{i k, j} g_{p l} g_{q m}+g_{i k} g_{p l, j} g_{q m}+g_{i k} g_{p l} g_{q m, j}\right) \\
& =\frac{1}{6}\left(e^{i p q} e^{k l m}+e^{p i q} e^{l k m}+e^{q p i} e^{m l k}\right) g_{i k, j} g_{p l} g_{q m} \\
g_{, j} & =\frac{1}{2} e^{i p q} e^{k l m} g_{p l} g_{q m} g_{i k, j}
\end{aligned}
$$

Novamente, passando da segunda para a terceira linha, os índices contraídos foram trocados de maneira conveniente. Na equação resultante, pode-se notar que os índices sobrescritos dos símbolos de permutação nas duas últimas equações sofreram permutações ímpares, quando comparadas à primeira equação.

Finalmente, substituindo os termos entre parentesis nas equações (A.21) pela equação (A.23) e substituindo o valor $J^{2}$ por $g$, chega-se a um resultado impor- 
tante:

$$
\Gamma_{j i}^{i}=\frac{1}{J} \frac{\partial J}{\partial \xi^{j}}
$$

É importante relembrar que a permutação e os tensores métricos possuem derivadas covariantes nulas (FLüGGE, 1972),

$$
\left.g^{i j}\right|_{k}=\left.g_{i j}\right|_{k}=\left.\epsilon^{i j k}\right|_{l}=\left.\epsilon_{i j k}\right|_{l}=0
$$

Entretanto, esta equação não implica que as derivadas parciais destes termos sejam zero também.

Os resultados acima permitem simplificações expressivas que podem ser feitas nas derivadas dos covariantes. Uma importância fundamental é a redução do operador divergente, que é obtido pela equação (A.24). Retornando a equação (A.8), primeira linha, temos que:

$$
\left.u^{i}\right|_{i}=u_{, i}^{i}+u^{k} \Gamma_{i k}^{i}=\frac{1}{J}\left(J u^{i}\right)_{, i}
$$

Uma forma alternativa é derivar a partir da equação (A.8), segunda linha, combinando com a equação (A.25), porém não é uma solução compacta e não irá focar nos tensores de segunda ordem, que são mais relevantes em nossas aplicações. Fora destas alternativas, a última linha da equação (A.9) apresenta que:

$$
\left.A^{i j}\right|_{j}=A_{, j}^{i j}+A^{l j} \Gamma_{j l}^{i}+A^{i l} \Gamma_{j l}^{j}=\frac{1}{J}\left(J A^{i j}\right)_{, j}+A^{l j} \Gamma_{j l}^{i}
$$

As equações (A.26) e (A.27) são bem conhecidas na literatura e outras identidades necessárias para a aplicação proposta neste trabalho podem também serem provadas. O primeiro caso, envolve a equação (A.18):

$$
\begin{aligned}
\beta_{r^{\prime}}^{s}\left(\beta_{n}^{r^{\prime}} u^{n}\right)_{, k} & =\beta_{r^{\prime}}^{s} \beta_{n}^{r^{\prime}}\left(u_{, k}^{n}\right)+u^{n} \beta_{r^{\prime}}^{s}\left(\beta_{n}^{r^{\prime}}\right)_{, k} \\
& =\delta_{n}^{s}\left(u_{, k}^{n}\right)+u^{n} \Gamma_{k n}^{s} \\
& =\left.u^{s}\right|_{k}
\end{aligned}
$$

que proporciona condições de expressar a derivada do covariante em termos do operador transformada e de derivadas parciais. Da mesma maneira, pode-se 
obter a partir a equação (A.19) a seguinte expressão:

$$
\beta_{s}^{r^{\prime}}\left(\beta_{r^{\prime}}^{n} u_{n}\right)_{, k}=\left.u_{s}\right|_{k}
$$

Na realidade, a equação (A.18) permite uma grande simplificação no divergente dos tensores de segunda ordem, em termos dos componentes contra variantes. A partir da equação (A.27), obtém que:

$$
\begin{aligned}
\left.A^{i j}\right|_{j} & =\frac{1}{J}\left(J A^{i j}\right)_{, j}+A^{l j} \Gamma_{j l}^{i} \\
& =\frac{1}{J}\left(J A^{i j}\right)_{, j}+A^{l j} \beta_{k^{\prime}}^{i}\left(\beta_{l}^{k^{\prime}}\right)_{, j} \\
\left.A^{i j}\right|_{j} & =\frac{1}{J} \beta_{k^{\prime}}^{i}\left(\beta_{l}^{k^{\prime}} J A^{l j}\right)_{, j}
\end{aligned}
$$

A última linha da equação (A.30) pode ser vista como uma equação para recuperar a primeira linha, diferenciando em relação a $\xi^{j}$

Os resultados acima, em particular as equações (A.26) a (A.30), serão usadas para se derivar as equações de Navier Stokes em sua forma reduzida.

\section{A.3 Equações de Navier Stokes na forma reduzida}

Para simplificar a análise, cada equação dos conjuntos (A.6) e (A.7) será abordada separadamente. Iniciando pela equação da continuidade, substitui-se a equação (A.26) no divergente desde que os termos métricos não dependam do tempo, o que é o caso de nossas aplicações:

$$
\partial_{o}(J \rho)+\left(J \rho u^{k}\right)_{, k}=0
$$

Assim, a expressão (A.31) é a equação da continuidade em sua forma reduzida.

A equação do momento necessita de uma consideração mais cuidadosa, iniciando a partir da equação (A.30) que substituirá os termos divergentes. Assim, a equação poderá ser multiplicada por $J \beta_{i}^{r^{\prime}}$ - que é independente do tempo, apesar da dependência em $\xi^{k}$. 


$$
\begin{aligned}
\partial_{o}\left(\rho u^{i}\right)+\left.\left(\rho u^{i} u^{j}\right)\right|_{j}+\left.g^{i j} p\right|_{j} & =\left.\tau^{i j}\right|_{j} \\
\partial_{o}\left(\rho u^{i}\right)+\frac{1}{J} \beta_{k^{\prime}}^{i}\left(\beta_{l}^{k^{\prime}} J u^{l} u^{j}\right)_{, j}+g^{i j} p_{, j} & =\frac{1}{J} \beta_{k^{\prime}}^{i}\left(\beta_{l}^{k^{\prime}} J \tau^{l j}\right)_{, j} \\
\partial_{o}\left(J \rho u^{r^{\prime}}\right)+\delta_{k^{\prime}}^{r^{\prime}}\left(J u^{k^{\prime}} u^{j}\right)_{, j}+J \beta_{i}^{r^{\prime}} g^{i p} \beta_{p}^{n^{\prime}} \beta_{n^{\prime}}^{j} p_{, j} & =\delta_{k^{\prime}}^{r^{\prime}}\left(\beta_{l}^{k^{\prime}} J \tau^{l j}\right)_{, j} \\
\partial_{o}\left(J \rho u^{r^{\prime}}\right)+\left[J \beta_{n^{\prime}}^{j}\left(u^{r^{\prime}} u^{n^{\prime}}+g^{r^{\prime} n^{\prime}} p\right)\right]_{, j} & =\left(\beta_{l}^{r^{\prime}} J \tau^{l j}\right)_{, j}
\end{aligned}
$$

Nesta equação, foi usada a identidade $\beta_{p}^{n^{\prime}} \beta_{n^{\prime}}^{j}=\delta_{p}^{j}$ da equação (A.14) e o fato do delta de Kronecker ser independente do espaço e tempo. A mesma propriedade vale para o tensor métrico nas coordenadas cartesianas, desde que seja dado por: $g^{r^{\prime} n^{\prime}} \equiv \delta_{n^{\prime}}^{r^{\prime}}$

Antes de prosseguir com a equação (A.32), é interessante simplificar a expressão do tensor das tensões. Ele pode ser obtido a partir da substituição dos operadores correspondentes nas equações (A.26) e (A.28)

$$
\begin{aligned}
\tau^{l j} & =\mu\left[\left(\left.u^{l}\right|^{j}+\left.u^{j}\right|^{l}\right)-\left.\frac{2}{3} g^{l j} u^{k}\right|_{k}\right] \\
& =\mu\left[\beta_{s^{\prime}}^{l}\left(\beta_{n}^{s^{\prime}} u^{n}\right)_{, p} g^{p j}+\beta_{s^{\prime}}^{j}\left(\beta_{n}^{s^{\prime}} u^{n}\right)_{, p} g^{p l}-\frac{2 g^{l j}}{3 J}\left(J u^{p}\right)_{, p}\right]
\end{aligned}
$$

e multiplicando o resultado por $J \beta_{l}^{r^{\prime}}$, chega-se a:

$$
\begin{aligned}
J \beta_{l}^{r^{\prime}} \tau^{l j} & =\mu\left[J\left(u^{r^{\prime}}\right)_{, p} g^{p j}+J \beta_{s^{\prime}}^{j}\left(u^{s^{\prime}}\right)_{, p} \beta_{l}^{r^{\prime}} g^{p l}-\frac{2 \beta_{l}^{r^{\prime}} g^{l j}}{3}\left(J u^{p}\right)_{, p}\right] \\
& =\mu\left[J\left(u^{r^{\prime}}\right)_{, p} \beta_{n^{\prime}}^{p} g^{n^{\prime} s^{\prime}} \beta_{s^{\prime}}^{j}+J \beta_{s^{\prime}}^{j}\left(u^{s^{\prime}}\right)_{, p} \beta_{n^{\prime}}^{p} g^{r^{\prime} n^{\prime}}-\frac{2 g^{r^{\prime} s^{\prime}} \beta_{s^{\prime}}^{j}}{3}\left(J \beta_{n^{\prime}}^{p} u^{n^{\prime}}\right)_{, p}\right] \\
& =\mu\left[J \beta_{s^{\prime}}^{j}\left(u^{r^{\prime}{ }_{n}^{\prime}} g^{n^{\prime} s^{\prime}}+u_{{ }^{\prime}{ }^{\prime}} g^{r^{\prime} n^{\prime}}-\frac{2}{3} u_{{ }^{n^{\prime}}{ }^{\prime}} g^{r^{\prime} s^{\prime}}\right)\right] \\
J \beta_{l}^{r^{\prime}} \tau^{l j} & =J \beta_{s^{\prime}}^{j} \tau^{r^{\prime} s^{\prime}}
\end{aligned}
$$

Como esperado, o resultado é uma função do tensor das tensões em coordenadas cartesianas. É possível também derivar este resultado com uma aplicação conveniente do operador transformada a partir do lado direito da equação (A.32), porém o procedimento foi feito de outra maneira, para se obter uma descrição mais detalhada da equivalência das formas reduzida e extendida.

Finalmente, substituindo a equação (A.34) pelo seu termo correspondente na 
equação (A.32), e utilizando-se da equação (A.14), chega-se a:

$$
\partial_{o}\left(J \rho u^{r^{\prime}}\right)+J \beta_{n^{\prime}}^{j}\left(u^{r^{\prime}} u^{n^{\prime}}+g^{r^{\prime} n^{\prime}} p-\tau^{r^{\prime} n^{\prime}}\right)_{, j}=0
$$

que corresponde a forma reduzida da equação de momento.

Utilizando-se da mesma abordagem com a equação de energia, temos que:

$$
\begin{aligned}
\partial_{o}\left(\rho e_{o}\right)+\left.\left(\rho h_{o} u^{i}-\left.g^{i j} k T\right|_{j}-\tau^{i j} u_{j}\right)\right|_{i} & =0 \\
\partial_{o}\left(J \rho e_{o}\right)+\left[J\left(\rho u^{i} h_{o}-k g^{i j} T_{, j}-\tau^{i j} u_{j}\right)\right]_{, i} & =0 \\
\partial_{o}\left(J \rho e_{o}\right)+J \beta_{j^{\prime}}^{i}\left(\rho u^{j^{\prime}} h_{o}-k g^{j^{\prime} k^{\prime}} T_{k^{\prime}}-\tau^{j^{\prime} k^{\prime}} u_{k^{\prime}}\right)_{, i} & =0
\end{aligned}
$$

onde a equação (A.14) foi usada novamente para se obter a forma reduzida.

Por fim, rearranjando as equações que governam o escoamento (A.31), (A.35) e (A.36), chega-se ao seguinte resultado:

$$
\begin{cases}\partial_{o}(J \rho)+J \beta_{q^{\prime}}^{k}\left(\rho u^{q^{\prime}}\right)_{, k} & =0 \\ \partial_{o}\left(J \rho u^{r^{\prime}}\right)+J \beta_{n^{\prime}}^{j}\left(u^{r^{\prime}} u^{n^{\prime}}+g^{r^{\prime} n^{\prime}} p-\tau^{r^{\prime} n^{\prime}}\right)_{, j} & =0 \\ \partial_{o}\left(J \rho e_{o}\right)+J \beta_{j^{\prime}}^{i}\left(\rho u^{j^{\prime}} h_{o}-k g^{j^{\prime} k^{\prime}} T_{, k^{\prime}}-\tau^{j^{\prime} k^{\prime}} u_{k^{\prime}}\right)_{, i}=0\end{cases}
$$

que representa a forma reduzida das equações de Navier Stokes generalizadas, como pode ser visto na literatura (JAMESON; MARTINELLI; PIERCE, 1998). É importante notar que essas equações envolvem ambos os sistemas de coordenadas, cartesiada e transformada.

\section{A.4 Escoamentos Incompressíveis}

As aplicações que foram consideradas neste trabalho referem-se a apenas escoamentos incompressíveis e isotérmicos. As equações que governam o escoamento para este caso em particular podem ser simplificadas, iniciando-se a partir das equações (A.6)-(A.7) e (A.37).

Para problemas isotérmicos, a equação de conservação de energia se reduz a conservação de energia mecânica. Como é conhecida da literatura, a última equação é o produto da equação do momento linear com a velocidade do escoamento e que para escoamentos incompressíveis, a equação da continuidade impõe 
divergência nula no campo de velocidades.

$$
\left.u^{i}\right|_{i}=0
$$

Como resultado, o tensor das tensões (A.7) se reduz a:

$$
\tau^{i j}=\mu\left(\left.u^{i}\right|^{j}+\left.u^{j}\right|^{i}\right)
$$

No cálculo da divergência de $\tau$, como aparece na equação do momento, outra simplificação é facilmente visualizada:

$$
\begin{aligned}
\left.\tau^{i j}\right|_{j} & =\mu\left(\left.\left.u^{i}\right|^{j}\right|_{j}+\left.\left.u^{j}\right|^{i}\right|_{j}\right) \\
& =\left.\left.\mu u^{i}\right|^{j}\right|_{j}
\end{aligned}
$$

desde que a ordem das derivadas do covariante possam ser trocadas, como discutido acima, e isto implica em $\left.\left.u^{j}\right|_{j}\right|^{i}=0$.

Realizando estas simplificações nas equações (A.6) e (A.7), chega-se a:

$$
\left\{\begin{aligned}
\left.u^{i}\right|_{i} & =0 \\
\partial_{o} u^{i}+\left.\left(u^{i} u^{j}+g^{i j} p v\right)\right|_{j} & =\left.\left.\nu u^{i}\right|^{j}\right|_{j}
\end{aligned}\right.
$$

A mesma simplificação reduz a equação (A.37) a:

$$
\left\{\begin{array}{r}
\left(J \beta_{q^{\prime}}^{k} u^{q^{\prime}}\right)_{, k}=0 \\
\partial_{o}\left(J u^{r^{\prime}}\right)+\left\{J \beta_{n^{\prime}}^{j}\left[u^{r^{\prime}} u^{n^{\prime}}+g^{r^{\prime} n^{\prime}} p v-\nu\left(u^{r^{\prime}}\right)_{, p^{\prime}} g^{p^{\prime} n^{\prime}}\right]\right\}_{, j}=0
\end{array}\right.
$$

onde os termos métricos foram levados para dentro da derivada por conveniência algébrica. Neste caso, a equação (A.14) também foi utilizada.

\section{A.5 Conclusões}

A derivação da forma reduzida (Eq. A.37) a partir da versão extendida (Eq. A.6) não envolve considerações relativas a mecânica dos fluídos. Pelo contrário, o procedimento é puramente algébrico e assim pode-se afirmar sua equivalência matemática na modelagem física do problema.

A condição de incompressibilidade que foi imposta, na forma extendida (Eq. A.6) e na forma reduzida (Eq. A.37), para se obter as equações correspondentes 
da classe de escoamento - ou seja, (A.41) e (A.42), respectivamente. Porém ressalta-se que nenhuma consideração física foi feita para se chegar a esse resultado, sendo também uma dedução puramente algébrica como entre as equações (A.6) e (A.37).

Comparando a forma reduzida com suas partes extendidas, percebe-se claramente que as anteriores são mais simples que as últimas, e isso justifica que a forma reduzida prevalece na literatura. Adicionalmente, é importante ressaltar que a forma reduzida tem alguns termos métricos apresentados explicitamente e isto facilita o cálculo de seu gradiente.

Com vários pontos a favor da forma reduzida, a única vantagem pertinente da forma extendida é sua facilidade quando se aplica o teorema de Gauss, para se chegar na forma generalizada.

$$
\left.\int_{\mathcal{D}} u^{k}\right|_{k} \mathrm{~d} V=\oint_{\partial \mathcal{D}} u^{k} n_{k} \mathrm{~d} A
$$

Esta etapa é fundamental para o desenvolvimento do problema adjunto. 


\section{APÊNDICE B - RELAÇÃO ENTRE OS ELEMENTOS DE ÁREA DOS ESPAÇOS FÍSICO E TRANFORMADO}

Tomando um superfície aleatória no $\mathbf{R} 3$, denominada $S^{\prime}$ e dada em coordenadas cartesianas por $x^{3^{\prime}}=x^{3^{\prime}}\left(x^{1^{\prime}}, x^{2^{\prime}}\right)$, o elemento de área da superfície pode ser dado por (KAPLAN, 1971; PISKUNOV, 1968):

$$
\mathrm{d} S^{\prime}=\sqrt{1+\left(\frac{\partial x^{3^{\prime}}}{\partial x^{1^{\prime}}}\right)^{2}+\left(\frac{\partial x^{3^{\prime}}}{\partial x^{2^{\prime}}}\right)^{2}} \mathrm{~d} x^{1^{\prime}} \mathrm{d} x^{2^{\prime}}
$$

Além disso, assumindo que $S^{\prime}$ pode ser mapeado como um plano constante $S$ no espaço transformado $\left(S^{\prime} \Leftrightarrow S: \xi^{3}=0\right)$ e que a transformação é definida como na equação A.2, temos que:

$$
J=\operatorname{det}\left(\beta_{i}^{j^{\prime}}\right)=\frac{\partial\left(x^{1^{\prime}}, x^{2^{\prime}}, x^{3^{\prime}}\right)}{\partial\left(\xi^{1}, \xi^{2}, \xi^{3}\right)}
$$

Então o elemento de área na superfície deste sistema em particular será dado por $\mathrm{d} S=\mathrm{d} \xi^{1} \mathrm{~d} \xi^{2}$. Sob tais circunstâncias, pode-se colocar a relação entre os elementos de área da seguinte forma:

$$
\begin{aligned}
\left|\frac{\mathrm{d} S^{\prime}}{\mathrm{d} S}\right| \mathrm{d} S & =\sqrt{1+\left(\frac{\partial x^{3^{\prime}}}{\partial x^{1^{\prime}}}\right)^{2}+\left(\frac{\partial x^{3^{\prime}}}{\partial x^{2^{\prime}}}\right)^{2}} \frac{\partial\left(x^{1^{\prime}}, x^{2^{\prime}}\right)}{\partial\left(\xi^{1}, \xi^{2}\right)} \mathrm{d} \xi^{1} \mathrm{~d} \xi^{2} \\
\left|\frac{\mathrm{d} S^{\prime}}{\mathrm{d} S}\right| & =\sqrt{1+\left(\frac{\partial x^{3^{\prime}}}{\partial x^{1^{\prime}}}\right)^{2}+\left(\frac{\partial x^{3^{\prime}}}{\partial x^{2^{\prime}}}\right)^{2}} \frac{\partial\left(x^{1^{\prime}}, x^{2^{\prime}}\right)}{\partial\left(\xi^{1}, \xi^{2}\right)}
\end{aligned}
$$

O Jacobiano 2-D no lado direito da equação B.3 pode ser reconhecido como o determinante que é obtido substituindo a terceira linha pela terceira coluna do Jacobiano de transformação (B.2). Finalmente, fazendo uso da regra da cadeia e 
das definições de $\beta_{j^{\prime}}^{i}$ e $\beta_{i}^{j^{\prime}}$ da equação (A.2), temos que:

$$
\left|\frac{\mathrm{d} S^{\prime}}{\mathrm{d} S}\right|=\sqrt{1+\left(\frac{\beta_{1^{\prime}}^{3}}{\beta_{3^{\prime}}^{3}}\right)^{2}+\left(\frac{\beta_{2^{\prime}}^{3}}{\beta_{3^{\prime}}^{3}}\right)^{2}} \frac{\operatorname{Cof}\left(\beta_{3}^{3^{\prime}}\right)}{\beta_{3}^{3^{\prime}}}
$$

onde o símbolo Cof $\left(\beta_{3}^{3^{\prime}}\right)$ indica o cofator do elemento $\beta_{3}^{3^{\prime}}$. Assim, a equação B.4 é válida para $\beta_{3}^{3^{\prime}}$ e $\beta_{3}^{3^{\prime}}$ não nulos.

Para os problemas bidimensionais, pode-se considerar um contorno que é dado por $S^{\prime}: x^{2^{\prime}}=x^{2^{\prime}}\left(x^{1^{\prime}}\right)$ nas coordenadas cartesianas. Assumindo que tal curva pode ser mapeada como uma linha de coordenadas constantes no espaço transformado, pode-se escrever que:

$$
S^{\prime} \Leftrightarrow S: \xi^{2}\left(x^{1^{\prime}}, x^{2^{\prime}}\right)=0 \Leftrightarrow\left\{\begin{array}{l}
x^{1^{\prime}}=x^{1^{\prime}}\left(\xi^{1}, 0\right) \\
x^{2^{\prime}}=x^{2^{\prime}}\left(\xi^{1}, 0\right)
\end{array}\right.
$$

Neste caso, um elemento infinitesimal do contorno é dado (em coordenadas cartesianas) por:

$$
\mathrm{d} S^{\prime}=\sqrt{1+\left(\frac{\partial x^{2^{\prime}}}{\partial x^{1^{\prime}}}\right)^{2}} \mathrm{~d} x
$$

e, no espaço transformado, o elemento de contorno é dado por $\mathrm{d} S=\mathrm{d} \xi^{1}$. Então, seguindo o mesmo procedimento acima, obtém-se que:

$$
\begin{aligned}
\left|\frac{\mathrm{d} S^{\prime}}{\mathrm{d} \xi^{1}}\right| \mathrm{d} \xi^{1} & =\sqrt{1+\left(\frac{\partial x^{2^{\prime}} / \partial \xi^{1}}{\partial x^{1^{\prime}} / \partial \xi^{1}}\right)^{2}} \frac{\partial x^{1^{\prime}}}{\partial \xi^{1}} \mathrm{~d} \xi \\
\left|\frac{\mathrm{d} S^{\prime}}{\mathrm{d} \xi^{1}}\right| & =\sqrt{\left(\frac{\partial x^{1^{\prime}}}{\partial \xi^{1}}\right)^{2}+\left(\frac{\partial x^{2^{\prime}}}{\partial \xi^{1}}\right)^{2}}=\sqrt{g_{11}}
\end{aligned}
$$

onde o resultado coincide com um elemento covariante particular do tensor métrico $g_{i j}$. 